\title{
Two Exotic Holonomies in Dimension Four, Path Geometries, and Twistor Theory
}

by

\author{
ROBERT L. BRYANT*
}

\section{Table of Contents}
$\S \mathbf{0}$. Introduction
$\S 1$. The Holonomy of a Torsion-Free Connection
$\S 2$. The Structure Equations of $H_{3}$ - and $G_{3}$-structures
$\S 3$. The Existence Theorems
§4. Path Geometries and Exotic Holonomy
$\S 5$. Twistor Theory and Exotic Holonomy
$\S 6$. Epilogue

\section{$\S$ 0. Introduction}

Since its introduction by Élie Cartan, the holonomy of a connection has played an important role in differential geometry. One of the best known results concerning holonomy is Berger's classification of the possible holonomies of Levi-Civita connections of Riemannian metrics. Since the appearance of Berger [1955], much work has been done to refine his list of possible Riemannian holonomies. See the recent works by Besse [1987] or Salamon [1989] for useful historical surveys and applications of holonomy to Riemannian and algebraic geometry.

Less well known is that, at the same time, Berger also classified the possible pseudoRiemannian holonomies which act irreducibly on each tangent space. The intervening years have not completely resolved the question of whether all of the subgroups of the general linear group on his pseudo-Riemannian list actually occur as holonomy groups of pseudo-Riemannian metrics, but, as of this writing, only one class of examples on his list remains in doubt, namely $\mathrm{SO}^{*}(2 p) \subset \mathrm{GL}(4 p)$ for $p \geq 3$.

Perhaps the least-known aspect of Berger's work on holonomy concerns his list of the possible irreducibly-acting holonomy groups of torsion-free affine connections. (Torsionfree connections are the natural generalization of Levi-Civita connections in the metric case.) Part of the reason for this relative obscurity is that affine geometry is not as well known or widely used as Riemannian geometry and global results are generally lacking. However, another reason is perhaps that Berger's list is incomplete in the affine case; it

\footnotetext{
* partially supported by NSF grants DMS-8352009 and DMS-8905207
} 
omits a finite number of possibilities. In fact, just how many are missing from this list is not known. We shall refer to the missing entries as the list of exotic holonomies.

In this paper, we produce two examples which show that, in fact, the list of exotic holonomies is non-empty. Moreover, we show that the geometry of connections with these holonomies is related to classical work on the geometry of ODE in terms of "path geometries" and to the modern theory of twistors as initiated by Penrose and his co-workers.

In $\S 1$, we give a brief discussion of the two criteria which Berger used to compile his lists. We then apply these criteria to a simple collection of subgroups: For each $d \geq 1$ we can regard $\mathrm{SL}(2, \mathbb{R})$ as a subgroup $H_{d} \subset \mathrm{GL}(d+1, \mathbb{R})$ via the (unique) $(d+1)$-dimensional irreducible representation of $\operatorname{SL}(2, \mathbb{R})$. Moreover, if we let $G_{d} \subset \operatorname{GL}(d+1, \mathbb{R})$ denote the centralizer of $H_{d}$, then $G_{d}$ may be regarded as a representation of $\operatorname{GL}(2, \mathbb{R})$. We show that $H_{3}$ and $G_{3}$ satisfy Berger's criteria even though they do not preserve any non-trivial symmetric quadratic form (even up to a conformal factor) and do not appear on Berger's list of possible holonomies of torsion-free connections on 4-manifolds. Thus, these are candidates for exotic holonomy groups.

In $\S 2$, we investigate the structure equations for torsion-free connections on 4-manifolds whose holonomy is (conjugate to) a subgroup of either $H_{3}$ or $G_{3}$. We demonstrate the basic relationship between connections with these holonomies and torsion-free $H_{3^{-}}$and $G_{3}$-structures on 4-manifolds. Of course, this is quite straightforward. However, some interesting things turn up. For example, it is a consequence of our calculations in $\S 2$ that, for $k \geq 3$, the $k^{t h}$ covariant derivatives of the curvature tensor of a connection with holonomy $H_{3}$ are expressible as universal polynomials in the curvature tensor and its first two covariant derivatives. Generally, however, this section serves as a repository of the formulas (some, unfortunately, quite elaborate) needed in later sections.

In $\S 3$, we prove that connections with either of these holonomies do, indeed, exist. We first show that there is a differential system with independence condition whose space of integral manifolds can be interpreted as the space of diffeomorphism classes of 4-manifolds endowed with certain "non-degenerate" torsion-free connections with holonomy $G_{3}$. (For the meaning of "non-degenerate", see $\S 3$.) We then show that this system is involutive and conclude that, in the real analytic category, the space of non-degenerate torsion-free $G_{3}$-structures modulo diffeomorphism depends on four functions of three variables. Of course, our main tool is the Cartan-Kähler Theorem. The reader who is familiar with Bryant [1987] should be cautioned that the differential system constructed in this paper is not of the same sort constructed in that paper. In fact, our approach is more closely related to that of Cartan [1943]. It is worth remarking that Theorem 3.3 is purely an existence result and does not seem to be of much help in constructing explicit examples.

We then turn our attention to the geometry of torsion-free $H_{3}$-structures. It is in this section that the theory exhibits a remarkable serendipity and the most unexpected behavior. The upshot of our work is that a torsion-free $H_{3}$-structure always has at least a one-parameter local symmetry group, but (except for the flat structure) is never locally symmetric. Moreover, the (non-Hausdorff) "moduli space" of such structures is essentially composed of a disjoint union of a two-dimensional space, two one-dimensional spaces, and four points. 
To see how unusual this is, consider that, in all previously known cases of an irreducibly acting subgroup $G \subset \mathrm{GL}(n, \mathbb{R})$ which can be holonomy, either there exist only locally symmetric examples or else there exist connections with holonomy $G$ which have no local symmetries and, moreover, there exist at least an "arbitrary function's worth" of inequivalent connections with holonomy $G$. This "rigid-but-not-symmetric" behavior of $H_{3}$-holonomy connections is perhaps the most remarkable aspect of this entire paper.

In $\S 4$, we begin to construct the bridge between the purely differential geometric treatment in the previous sections and the twistor-theoretic treatment given in $\S 5$. In particular, we show how a torsion-free $G_{3}$-structure gives rise to a (contact) path geometry in the sense of Cartan. This geometry interprets the original 4-manifold as the space of solutions of a fourth order ODE for one function of one variable.

This aspect of the theory fits into a program of "geometrizing" classical oDE as envisioned in Cartan [1938] and Chern [1940]. Cartan had observed that his method of producing differential invariants of geometric structures, known as the method of equivalence, could be used to integrate certain classes of ODE. From the examples he knew, he then abstracted a general approach which led him to define certain classes of equations which he called classes $C$.

It turns out that the class consisting of the fourth order ODE which arise from torsionfree $G_{3}$-structures constitutes an instance of classe $C$. Although we do not pursue this in this paper, it can be shown that, in an appropriate sense, this class is the largest classe $C$ among the fourth order ODE. In fact, it was in connection with the larger problem of understanding Cartan's classes $C$ that the author was led to consider torsion-free $G_{3^{-}}$ structures in the first place.

In more modern language, we use a torsion-free $G_{3}$-structure to construct a double fibration $N$, one base of which is the original 4-manifold $M$ on which the connection is defined while the other base is the 3-dimensional contact manifold $Y$ of "totally geodesic null surfaces" in $M$ where the notion of "nullity" is constructed out of a quartic form associated to the $G_{3}$-holonomy connection. We show that this double fibration is nondegenerate and apply the method of equivalence to determine the conditions for a given double fibration to arise from a torsion-free $G_{3}$-structure. These conditions take the form of requiring that certain invariants, herein called the primary and secondary invariants, vanish.

In $\S 5$, we describe the relationship of this path geometry with twistor theory proper by moving from the real category to the holomorphic category. In this transition, the space $Y$ is replaced by a complex contact manifold $\mathcal{Y}$ and the points of $\mathcal{M}$, a "complexification" of $M$, are interpreted as rational contact curves in $\mathcal{Y}$ with normal bundle $\mathcal{O}(2) \oplus \mathcal{O}(2)$. We show, by methods analogous to those in Hitchin [1982], how the holomorphic geometry encodes the conditions of vanishing of the primary and secondary invariants of the double fibration and hence gives rise to torsion-free "complexified" $G_{3}$-structures.

We close this section by applying the twistor description to show how one can, in principle, construct many explicit examples of torsion-free $G_{3}$-structures by considering the geometry of rational holomorphic curves in $\mathbb{C P}^{2}$. The simplest example, that of smooth conics passing through a fixed point in $\mathbb{C P}^{2}$, corresponds to the unique non-flat homoge- 
neous torsion-free $H_{3}$-structure. We also give a (very brief) discussion of how the KodairaSpencer deformation theory of pseudo-group structures on complex manifolds might be used to "construct" deformations of torsion-free $G_{3}$-structures.

Finally, in $\S 6$, we point out some of the interesting questions which this investigation has raised.

It would be remiss of this author indeed if he did not comment on the method of calculation employed in this paper. The fact is that the vast majority of these calculations were carried out on computers using the symbolic manipulation program MAPLE. Many of the formulas, if written out in full, would contain literally hundreds of terms and would be utterly incomprehensible. This is particularly true of the calculations in $\S 3$ and $\S 4$. This presented a problem for the author: How can one explain the essential points without being able to demonstrate them by (sample) calculation or without the reader having the ability to check the calculations personally?

For example, the essential point in the analysis of torsion-free $H_{3}$-structures is the existence of three remarkable identities for which the author has been able to find no conceptual justification. These identities were found by brute force and seem to just happen to be true. For anyone to find or verify them by hand seems extremely unlikely.

Thus, the author settled on the following strategy: Give the reader enough detail to understand the structure of the argument and to form an opinion as to the reasonableness of the claims and then make available thoroughly documented MAPLE files which will allow the reader to check the calculations by computer if so inclined. This the author has done. Anyone wishing to obtain copies of these files is encouraged to contact the author either by corporeal or electronic mail at the addresses provided.

Since the calculations in this paper make extensive use of the representation theory of $\mathrm{SL}(2, \mathbb{R})$, particularly an explicit version of the Clebsch-Gordan formula, we will give a short account of it here to establish notation. A good reference for proofs is Humphreys [1972], though our notation is different.

Let $x$ and $y$ denote two indeterminates and let $\mathbb{R}[x, y]$ denote the polynomial ring with real coefficients generated by $x$ and $y$. Let $\operatorname{SL}(2, \mathbb{R})$ act on $\mathbb{R}[x, y]$ in the usual way via unimodular linear substitutions in $x$ and $y$. The infinitesimal version of this action is generated by the Lie algebra $\mathcal{D}$ spanned by the three derivations

$$
X=x \frac{\partial}{\partial y}, \quad H=x \frac{\partial}{\partial x}-y \frac{\partial}{\partial y}, \quad Y=y \frac{\partial}{\partial x} .
$$

Let $\mathcal{V}_{d} \subset \mathbb{R}[x, y]$ denote the subspace consisting of homogeneous polynomials of degree $d$. Then it is well-known that $\mathcal{V}_{d}$ is an irreducible $\mathrm{SL}(2, \mathbb{R})$-module and that every finitedimensional, irreducible $\operatorname{SL}(2, \mathbb{R})$-module is isomorphic to $\mathcal{V}_{d}$ for some $d \geq 0$.

It will occasionally be necessary to refer to a basis of $\mathcal{V}_{d}$. For this purpose, we use the standard basis given by the monomials $x^{(d-j) / 2} y^{(d+j) / 2}$ where $j$ ranges over the integers which lie between $-d$ and $d$ and are congruent to $d$ modulo 2. The $x^{(d-j) / 2} y^{(d+j) / 2}$ coefficient of an arbitrary element $v \in \mathcal{V}_{d}$ will be denoted by $v_{-j}$. Thus, $v$ has the expansion $v=v_{-d} x^{d}+v_{-d+2} x^{d-1} y+\cdots+v_{d} y^{d}$. 
The Clebsch-Gordan formula describes the irreducible decomposition of a tensor product of irreducible $\mathrm{SL}(2, \mathbb{R})$-modules:

$$
\mathcal{V}_{m} \otimes \mathcal{V}_{n}=\mathcal{V}_{|m-n|} \oplus \mathcal{V}_{|m-n|+2} \oplus \cdots \oplus \mathcal{V}_{m+n-2} \oplus \mathcal{V}_{m+n}
$$

We shall need an explicit formula for this decomposition which we now describe. For each $p \geq 0$, define the bilinear pairing $\langle,\rangle_{p}: \mathbb{R}[x, y] \times \mathbb{R}[x, y] \rightarrow \mathbb{R}[x, y]$ by

$$
\langle u, v\rangle_{p}=\frac{1}{p !} \sum_{k=0}^{p}(-1)^{k}\left(\begin{array}{l}
p \\
k
\end{array}\right) \frac{\partial^{p} u}{\partial x^{p-k} \partial y^{k}} \frac{\partial^{p} v}{\partial x^{k} \partial y^{p-k}} .
$$

For example, $\langle u, v\rangle_{0}=u v$ and $\langle u, v\rangle_{1}=u_{x} v_{y}-u_{y} v_{x}$.

It is easy to prove that $D\langle u, v\rangle_{p}=\langle D u, v\rangle_{p}+\langle u, D v\rangle_{p}$ for any $p \geq 0$ and any $D \in \mathcal{D}$. It follows that the pairings $\langle,\rangle_{p}$ are $\operatorname{SL}(2, \mathbb{R})$-equivariant. For $u \in \mathcal{V}_{m}$ and $v \in \mathcal{V}_{n}$, we have $\langle u, v\rangle_{p} \in \mathcal{V}_{m+n-2 p}$ and, moreover, the induced linear mapping $\mathcal{V}_{m} \otimes \mathcal{V}_{n} \rightarrow \mathcal{V}_{m+n-2 p}$ is clearly non-trivial for $0 \leq p \leq \min (m, n)$. Thus, these pairings give the non-trivial projections implicit in the Clebsch-Gordan formula.

The following identities are easily verified:

$$
\begin{gathered}
\langle u, v\rangle_{p}=(-1)^{p}\langle v, u\rangle_{p} \\
\left\langle u,\langle v, w\rangle_{1}\right\rangle_{1}-\left\langle v,\langle u, w\rangle_{1}\right\rangle_{1}=\left\langle\langle u, v\rangle_{1}, w\right\rangle_{1}
\end{gathered}
$$

In particular, note that $\langle,\rangle_{p}: \mathcal{V}_{p} \times \mathcal{V}_{p} \rightarrow \mathcal{V}_{0}=\mathbb{R}$ is non-trivial and hence is an $\operatorname{SL}(2, \mathbb{R})$ invariant symmetric or skew-symmetric form on $\mathcal{V}_{p}$ depending on whether $p$ is even or odd.

These pairings satisfy an enormous number of further identities which we shall not attempt to enumerate, though we have used them implicitly in our calculations. For example, a frequently used identity is

$$
\left\langle u,\langle v, w\rangle_{2}\right\rangle_{1}-\left\langle\langle u, v\rangle_{1}, w\right\rangle_{2}-\left\langle v,\langle u, w\rangle_{1}\right\rangle_{2}=v\langle u, w\rangle_{3}+w\langle u, v\rangle_{3}-\langle u, v w\rangle_{3} .
$$

However, a complete list of the identities that we have used in the course of our calculations would be so long that it would not be comprehensible or useful. Unfortunately, a systematic method of deriving these identities (other than brute force) is not known to the author.

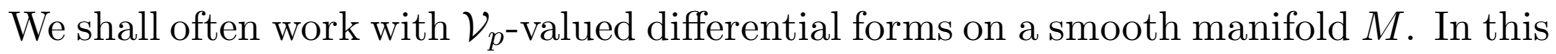
case, we simply extend the pairings $\langle,\rangle_{p}: \mathcal{V}_{m} \times \mathcal{V}_{n} \rightarrow \mathcal{V}_{m+n-2 p}$ as graded $\Omega^{*}(M)$-module pairings

$$
\langle,\rangle_{p}:\left(\Omega^{*}(M) \otimes \mathcal{V}_{m}\right) \times\left(\Omega^{*}(M) \otimes \mathcal{V}_{n}\right) \rightarrow\left(\Omega^{*}(M) \otimes \mathcal{V}_{m+n-2 p}\right) .
$$

In particular, note that if $\omega$ is a $\mathcal{V}_{m}$-valued $r$-form on $M$ and $\eta$ is a $\mathcal{V}_{n}$-valued $s$-form on $M$, then

$$
\langle\omega, \eta\rangle_{p}=(-1)^{r s+p}\langle\eta, \omega\rangle_{p} .
$$




\section{$\S 1$. The Holonomy of a Torsion-Free Connection}

Let $M^{n}$ be a smooth, connected, and simply connected $n$-manifold. (The assumption of simple connectivity is made for the sake of convenience. For the problems we wish to address, the more general case does not differ in any significant way.) Let $\mathcal{P}(M)$ denote the set of piecewise smooth paths $\gamma:[0,1] \rightarrow M$. For $x \in M$, let $\mathcal{L}_{x}(M) \subset \mathcal{P}(M)$ denote the set of $x$-based loops in $M$, namely those $\gamma \in \mathcal{P}(M)$ for which $\gamma(0)=\gamma(1)=x$.

Let $\nabla$ be a torsion-free affine connection on the tangent bundle of $M$. (In order to avoid confusion, we shall not follow the common practice of using the term symmetric as a synonym for torsion-free.) For each $\gamma \in \mathcal{P}(M)$, the connection $\nabla$ defines a linear isomorphism $P_{\gamma}: T_{\gamma(0)} M \rightarrow T_{\gamma(1)} M$ called parallel translation along $\gamma$. For each $x \in M$, we define the holonomy of $\nabla$ at $x$ to be the subset of $\mathrm{GL}\left(T_{x} M\right)$ given by

$$
H_{x}=\left\{P_{\gamma} \mid \gamma \in \mathcal{L}_{x}(M)\right\} \subset \mathrm{GL}\left(T_{x} M\right) .
$$

It is well known (see Kobayashi and Nomizu [1963]), that $H_{x}$ is a connected Lie subgroup of $\operatorname{GL}\left(T_{x} M\right)$ and that, for any $\gamma \in \mathcal{P}(M), \quad P_{\gamma}$ induces an isomorphism of $T_{\gamma(0)} M$ with $T_{\gamma(1)} M$ which identifies $H_{\gamma(0)}$ with $H_{\gamma(1)}$.

Choose an $x_{0} \in M$ and an isomorphism $\iota: T_{x_{0}} M \rightarrow V$, where $V$ is a fixed real vector space of dimension $n$. Then, because $M$ is connected, the conjugacy class of the subgroup $H \subset \mathrm{GL}(V)$ which corresponds under $\iota$ to $H_{x_{0}} \subset \mathrm{GL}\left(T_{x_{0}} M\right)$ is independent of the choice of $x_{0}$ or $\iota$. By abuse of language, we speak of $H$ as the holonomy of $\nabla$.

A basic question in the theory is this: Which (conjugacy classes of) subgroups $H \subset$ $\mathrm{GL}(V)$ can occur as the holonomy of some torsion-free connection $\nabla$ on some $n$-manifold $M$ ? Two necessary conditions on $H$ were derived by M. Berger [1955] in his thesis and we will now describe them.

Let us write $T, T^{*}$, etc. to denote the bundles $T M, T^{*} M$, etc. Let $R^{\nabla}$ denote the section of $\mathfrak{g l}(T) \otimes \Lambda^{2}\left(T^{*}\right)=T \otimes T^{*} \otimes \Lambda^{2}\left(T^{*}\right)$ which represents the curvature of $\nabla$. Let $\mathfrak{h} \subset \mathfrak{g l}(T)$ denote the sub-bundle whose fiber $\mathfrak{h}_{x}$ at $x \in M$ is the Lie algebra of $H_{x}(\nabla)$. Then $R^{\nabla}$ is a section of $\mathfrak{h} \otimes \Lambda^{2}\left(T^{*}\right)$. Moreover, because $\nabla$ is a torsion-free connection, the first Bianchi identity states that $R^{\nabla}$ takes values in the kernel bundle $\mathcal{K} \subset \mathfrak{g l}(T) \otimes \Lambda^{2}\left(T^{*}\right)$ of the short exact sequence

$$
0 \rightarrow \mathcal{K} \rightarrow \mathfrak{g l}(T) \otimes \Lambda^{2}\left(T^{*}\right) \rightarrow T \otimes \Lambda^{3}\left(T^{*}\right) \rightarrow 0,
$$

where the mapping $\mathfrak{g l}(T) \otimes \Lambda^{2}\left(T^{*}\right)=T \otimes T^{*} \otimes \Lambda^{2}\left(T^{*}\right) \rightarrow T \otimes \Lambda^{3}\left(T^{*}\right)$ is simply skewsymmetrization on the last three indices. In particular, $R^{\nabla}$ takes values in the bundle $\mathcal{K}(\mathfrak{h})=\mathcal{K} \cap\left(\mathfrak{h} \otimes \Lambda^{2}\left(T^{*}\right)\right.$ ). (Note that this intersection has constant rank because all of the subalgebras $\mathfrak{h}_{x}$ are conjugate under suitable identifications of the tangent spaces.)

Similarly, if we let $\nabla R^{\nabla}$ denote the covariant derivative of the curvature tensor, then $\nabla R^{\nabla}$ can be regarded as a section of the bundle $\mathfrak{g l}(T) \otimes \Lambda^{2}\left(T^{*}\right) \otimes T^{*}$. According to the second Bianchi identity, the condition that $\nabla$ be torsion-free implies that $\nabla R^{\nabla}$ has values in the kernel bundle $\mathcal{K}^{1} \subset \mathfrak{g l}(T) \otimes \Lambda^{2}\left(T^{*}\right) \otimes T^{*}$ of the short exact sequence

$$
0 \rightarrow \mathcal{K}^{1} \rightarrow \mathfrak{g l}(T) \otimes \Lambda^{2}\left(T^{*}\right) \otimes T^{*} \rightarrow \mathfrak{g l}(T) \otimes \Lambda^{3}\left(T^{*}\right) \rightarrow 0,
$$


where the mapping $\mathfrak{g l}(T) \otimes \Lambda^{2}\left(T^{*}\right) \otimes T^{*} \rightarrow \mathfrak{g l}(T) \otimes \Lambda^{3}\left(T^{*}\right)$ is again defined by skewsymmetrization on the last three indices. In particular, $\nabla R^{\nabla}$ takes values in the bundle $\mathcal{K}^{1}(\mathfrak{h})=\mathcal{K}^{1} \cap\left(\mathcal{K}(\mathfrak{h}) \otimes T^{*}\right)$.

This motivates the following definitions. For any $n$-dimensional vector space $V$, let us define $\mathcal{K}(V)$ and $\mathcal{K}^{1}(V)$ to be the vector spaces described by the exact sequences

$$
0 \rightarrow \mathcal{K}(V) \rightarrow \mathfrak{g l}(V) \otimes \Lambda^{2}\left(V^{*}\right) \rightarrow V \otimes \Lambda^{3}\left(V^{*}\right) \rightarrow 0
$$

and

$$
0 \rightarrow \mathcal{K}^{1}(V) \rightarrow \mathfrak{g l}(V) \otimes \Lambda^{2}\left(V^{*}\right) \otimes V^{*} \rightarrow \mathfrak{g l}(V) \otimes \Lambda^{3}\left(V^{*}\right) \rightarrow 0 .
$$

For any Lie subalgebra $\mathfrak{g} \subset \mathfrak{g l}(V)$, we define two vector spaces

$$
\mathcal{K}(\mathfrak{g})=\mathcal{K}(V) \cap\left(\mathfrak{g} \otimes \Lambda^{2}\left(V^{*}\right)\right)
$$

and

$$
\mathcal{K}^{1}(\mathfrak{g})=\mathcal{K}^{1}(V) \cap\left(\mathcal{K}(\mathfrak{g}) \otimes V^{*}\right)
$$

If $\mathfrak{g}^{\prime} \subset \mathfrak{g} \subset \mathfrak{g l}(V)$ is a pair of subalgebras of $\mathfrak{g l}(V)$, then we have $\mathcal{K}\left(\mathfrak{g}^{\prime}\right) \subset \mathcal{K}(\mathfrak{g})$ and $\mathcal{K}^{1}\left(\mathfrak{g}^{\prime}\right) \subset \mathcal{K}^{1}(\mathfrak{g})$. Moreover, if $\mathfrak{g}^{\prime} \subset \mathfrak{g l}(V)$ is any Lie subalgebra which is $\mathrm{GL}(V)$-conjugate to $\mathfrak{g}$, then there are isomorphisms $\mathcal{K}\left(\mathfrak{g}^{\prime}\right) \simeq \mathcal{K}(\mathfrak{g})$ and $\mathcal{K}^{1}\left(\mathfrak{g}^{\prime}\right) \simeq \mathcal{K}^{1}(\mathfrak{g})$. As we shall see, the existence of a mere abstract isomorphism $\mathfrak{g}^{\prime} \simeq \mathfrak{g}$ does not imply any relationship between $\mathcal{K}\left(\mathfrak{g}^{\prime}\right)$ and $\mathcal{K}(\mathfrak{g})$ or between $\mathcal{K}^{1}\left(\mathfrak{g}^{\prime}\right)$ and $\mathcal{K}^{1}(\mathfrak{g})$.

Recall that a connection $\nabla$ is said to be locally symmetric if the (local) $\nabla$-geodesic symmetry about each point of $M$ preserves $\nabla$. As is well known (see Kobayashi and Nomizu [1963]), the necessary and sufficient condition for $\nabla$ to be locally symmetric is that $\nabla$ be torsion-free and that $\nabla R^{\nabla}=0$.

By combining our discussion so far with the Ambrose-Singer Holonomy Theorem, the following result of Berger is now easily derived. Hence, we omit the proof.

Theorem 1.1 (Berger): Let $V$ be a real vector space of dimension $n$. Let $\mathfrak{g} \subset \mathfrak{g l}(V)$ be the Lie algebra of a Lie subgroup $G \subseteq \mathrm{GL}(V)$.

(i) If $\mathcal{K}(\mathfrak{g})=\mathcal{K}\left(\mathfrak{g}^{\prime}\right)$ for any proper subalgebra $\mathfrak{g}^{\prime} \subset \mathfrak{g}$, then $G$ is not (conjugate to) the holonomy of any torsion-free connection on any manifold $M$ of dimension $n$.

(ii) If $\mathcal{K}^{1}(\mathfrak{g})=0$, then any torsion-free connection whose holonomy is (conjugate to) a subgroup of $G$ is locally symmetric.

Since locally symmetric connections are also locally homogeneous, the study of locally symmetric connections can be reduced to the study of certain (non-trivial) problems in the theory of Lie algebras. Hence, we will not discuss the locally symmetric case any further.

It follows from Theorem 1.1 that two necessary conditions for a connected Lie subgroup $G \subset \mathrm{GL}(V)$ with Lie algebra $\mathfrak{g} \subset \mathfrak{g l}(V)$ to be the holonomy of a torsion-free connection which is not locally symmetric are, first, that $\mathcal{K}\left(\mathfrak{g}^{\prime}\right)$ be a proper subspace of $\mathcal{K}(\mathfrak{g})$ for 
every proper subalgebra $\mathfrak{g}^{\prime}$ of $\mathfrak{g}$, and, second, that $\mathcal{K}^{1}(\mathfrak{g}) \neq 0$. We shall refer to these two conditions as Berger's first and second criteria.

EXAMPLES: To get some feel for these criteria (and because we shall need the results in subsequent sections), let us compute some examples.

For $n \geq 1$, let $\mathcal{V}_{n}$ denote the irreducible $\mathrm{SL}(2, \mathbb{R})$-module of dimension $n+1$ described in $\S 0$. Let $\mathfrak{h}_{n} \subset \mathfrak{g l}\left(\mathcal{V}_{n}\right)$ denote the Lie algebra generated by the action of $\operatorname{SL}(2, \mathbb{R})$ on $\mathcal{V}_{n}$ and let $H_{n} \subset \mathrm{GL}\left(\mathcal{V}_{n}\right)$ denote the connected Lie subgroup whose Lie algebra is $\mathfrak{h}_{n}$. It is easy to see that $H_{n}$ is abstractly isomorphic to either $\operatorname{SL}(2, \mathbb{R})$ or $\operatorname{PSL}(2, \mathbb{R})$ accordingly as $n$ is odd or even. Using the Clebsch-Gordan formula and MAPLE, it is easy to compute the entries in Table I.

Table I

\begin{tabular}{|c|c|c|}
\hline$n$ & $\mathcal{K}\left(\mathfrak{h}_{n}\right)$ & $\mathcal{K}^{1}\left(\mathfrak{h}_{n}\right)$ \\
\hline \hline 1 & $\mathcal{V}_{2}$ & $\mathcal{V}_{1} \oplus \mathcal{V}_{3}$ \\
2 & $\mathcal{V}_{0} \oplus \mathcal{V}_{4}$ & $\mathcal{V}_{2} \oplus \mathcal{V}_{4} \oplus \mathcal{V}_{6}$ \\
3 & $\mathcal{V}_{2}$ & $\mathcal{V}_{3}$ \\
4 & $\mathcal{V}_{0}$ & 0 \\
$\geq 5$ & 0 & 0 \\
\hline
\end{tabular}

Similarly, let $\mathfrak{g}_{n} \subset \mathfrak{g l}\left(\mathcal{V}_{n}\right)$ denote the Lie algebra of dimension 4 which is generated by $\mathfrak{h}_{n}$ and the multiples of the identity mapping in $\mathfrak{g l}\left(\mathcal{V}_{n}\right)$, and let $G_{n} \subset \operatorname{GL}\left(\mathcal{V}_{n}\right)$ denote the connected subgroup whose Lie algebra is $\mathfrak{g}_{n}$. Applying the same methods as in the case of $\mathfrak{h}_{n}$, we can derive the entries of Table II.

Table II

\begin{tabular}{|c|c|c|}
\hline$n$ & $\mathcal{K}\left(\mathfrak{g}_{n}\right)$ & $\mathcal{K}^{1}\left(\mathfrak{g}_{n}\right)$ \\
\hline \hline 1 & $\mathcal{V}_{0} \oplus \mathcal{V}_{2}$ & $2 \mathcal{V}_{1} \oplus \mathcal{V}_{3}$ \\
2 & $\mathcal{V}_{0} \oplus \mathcal{V}_{2} \oplus \mathcal{V}_{4}$ & $2 \mathcal{V}_{2} \oplus 2 \mathcal{V}_{4} \oplus \mathcal{V}_{6}$ \\
3 & $\mathcal{V}_{2} \oplus \mathcal{V}_{4}$ & $\mathcal{V}_{1} \oplus \mathcal{V}_{3} \oplus \mathcal{V}_{5} \oplus \mathcal{V}_{7}$ \\
4 & $\mathcal{V}_{0}$ & 0 \\
$\geq 5$ & 0 & 0 \\
\hline
\end{tabular}

When $n \geq 5$, neither $\mathfrak{h}_{n}$ nor $\mathfrak{g}_{n}$ satisfy Berger's first criterion. In fact, since $\mathcal{K}\left(\mathfrak{g}_{n}\right)=$ $\mathcal{K}\left(\mathfrak{h}_{n}\right)=0$ for all $n \geq 5$, any torsion-free connection on an $(n+1)$-manifold whose holonomy is conjugate to any subgroup of $G_{n} \subset \mathrm{GL}\left(\mathcal{V}_{n}\right)$ is actually flat.

When $n=4$, since $\mathcal{K}\left(\mathfrak{g}_{4}\right)=\mathcal{K}\left(\mathfrak{h}_{4}\right)$ and $\mathfrak{h}_{4} \subset \mathfrak{g}_{4}$, it follows that $\mathfrak{g}_{4}$ does not satisfy Berger's first criterion.

On the other hand, $\mathfrak{h}_{4}$ does satisfy Berger's first criterion since, as is easily seen, $\mathcal{K}(\mathfrak{s})=0$ for any proper subalgebra $\mathfrak{s} \subset \mathfrak{h}_{4}$.

However, $\mathfrak{h}_{4}$ does not satisfy Berger's second criterion since $\mathcal{K}^{1}\left(\mathfrak{h}_{4}\right)=0$. Thus, any connection on a 5-manifold with holonomy conjugate to $H_{4} \subset \mathrm{GL}\left(\mathcal{V}_{4}\right)$ must be locally 
symmetric. In fact, it is easily shown that any such connection on a 5-manifold is locally equivalent to the canonical connection on one of the irreducible affine symmetric spaces $\mathrm{SL}(3, \mathbb{R}) / \mathrm{SO}(2,1)$ or $\mathrm{SU}(2,1) / \mathrm{SO}(2,1)$.

In the remaining dimensions, things are more interesting:

When $n=1$, we have $\mathfrak{g}_{1}=\mathfrak{g l}\left(\mathcal{V}_{1}\right)$ and $\mathfrak{h}_{1}=\mathfrak{s l}\left(\mathcal{V}_{1}\right)$. A glance at the tables shows that these two algebras satisfy Berger's criteria. The corresponding torsion-free connections on 2-manifolds are, respectively, the "generic" affine connections and the "generic" affine connections preserving a non-zero parallel area form. It is easy to see that non-locallysymmetric connections exist with holonomy either $H_{1}=\operatorname{SL}\left(\mathcal{V}_{1}\right)$ or $G_{1}=G L^{+}\left(\mathcal{V}_{1}\right)$.

When $n=2$, we have $\mathfrak{h}_{2}=\mathfrak{s o}\left(\mathcal{V}_{2}\right)$, the Lie algebra of linear transformations which preserve the $\operatorname{SL}(2, \mathbb{R})$-invariant (indefinite) quadratic form $\langle,\rangle_{2}$ on $\mathcal{V}_{2}$, and $\mathfrak{g}_{2}=\mathfrak{c o}\left(\mathcal{V}_{2}\right)$, the Lie algebra of linear transformations which preserve $\langle,\rangle_{2}$ up to a scalar multiple. Again, a glance at the tables shows that these two algebras satisfy Berger's criteria. In the case of $\mathfrak{h}_{2}$, the corresponding connections on 3-manifolds are the Levi-Civita connections of Lorentzian metrics. Of course, these do not have to be locally symmetric. A 3-manifold $M$ endowed with a torsion-free connection $\nabla$ whose holonomy is conjugate to a subgroup of $G_{2}=C O\left(\mathcal{V}_{2}\right)$ is known in the classical literature as a Weyl space, after Weyl's work on conformal geometry. It is easy to construct examples with holonomy $G_{2}$ which are not locally symmetric.

When $n=3$, the situation is the least understood. Note that both $\mathfrak{g}_{3}$ and $\mathfrak{h}_{3}$ satisfy Berger's second criterion. It is not difficult to show that they both satisfy Berger's first criterion. In the remaining sections of this paper, we will show that, for each of $H_{3}$ and $G_{3}$, a torsion-free connection with this holonomy does, in fact, exist. Moreover, as we shall show, these connections have interesting relationships with path geometry, twistor theory, and algebraic geometry.

We close this section with a short discussion of the general case. The original question can now be refined to the following one:

For which connected Lie subgroups $G \subset \mathrm{GL}(V)$ satisfying Berger's criteria do there exist torsion-free connections which are not locally symmetric and whose holonomy is (conjugate to) $G$ ?

To the author's knowledge, there is no $G \subset \mathrm{GL}(V)$ satisfying Berger's criteria which is known not to occur as the holonomy of any torsion-free connection which is not locally symmetric. Nevertheless, it seems too much to hope that Berger's necessary criteria are sufficient.

An obvious strategy for solving this problem is to first make a list of the subgroups of GL $(V)$ which satisfy Berger's criteria and then examine each case separately. A natural place to start is to classify those subgroups which, in addition, act irreducibly on $V$. Berger [1955] employed an extensive series of representation-theoretic calculations to make a list of (nearly) all of the connected groups $G \subset \mathrm{GL}(V)$ which act irreducibly on $V$ and which satisfy his two criteria. His list falls naturally into two parts (see Theorems 3 and 4 of Chapter III of Berger [1955]). 
The first part, the metric list, consists of the irreducibly acting $G$ which satisfy Berger's criteria and which fix some non-trivial symmetric quadratic form on $V$. Actually, Berger's metric list contains three spurious real forms of $\operatorname{Spin}(9, \mathbb{C})$ occurring as subgroups of $\mathrm{GL}(16, \mathbb{R})$ which do not satisfy his second criterion. Due to the efforts of several persons (see Salamon [1989] for an overview), every $G$ on the corrected list save one is now known to be the holonomy of some torsion-free connection which is not locally symmetric. (The remaining open case is that of $\mathrm{SO}^{*}(2 p) \subset \mathrm{GL}(4 p, \mathbb{R})$ where $p \geq 3$.)

The second part, the "non-metric" list, consists of all but a finite number of the irreducibly acting $G$ which satisfy Berger's criteria but which do not fix any non-trivial symmetric quadratic form on $V$. Unfortunately, because Berger gives no indication of the proof of his Theorem 4, we do not have an estimate of how many cases are missing from the non-metric list.

We shall refer to these missing subgroups as exotic. Since the subgroups $H_{3}$ and $G_{3}$ of $\operatorname{GL}\left(\mathcal{V}_{3}\right)$ do not appear on Berger's non-metric list, the set of exotic holonomies is non-empty.

Finally, let us note that, even among the groups which do appear on Berger's nonmetric list, there are many which are not known to be the holonomy group of any torsionfree connection which is not locally symmetric. Thus, the non-metric holonomy problem is far from being solved.

\section{The Structure Equations of $H_{3}$ - and $G_{3}$-structures}

Let us begin by discussing the geometry of $H_{3}$-structures on 4 -manifolds. Let $M$ be a 4-manifold and let $\pi: \mathcal{F} \rightarrow M$ be the $\mathcal{V}_{3}$-coframe bundle, i.e., each $u \in \mathcal{F}$ is a linear isomorphism $u: T_{\pi(u)} M \stackrel{\sim}{\rightarrow} \mathcal{V}_{3}$. Then $\mathcal{F}$ is naturally a principal right $\operatorname{GL}\left(\mathcal{V}_{3}\right)$-bundle over $M$ where the right action $R_{g}: \mathcal{F} \rightarrow \mathcal{F}$ is defined by $R_{g}(u)=g^{-1} \circ u$. A $\mathcal{V}_{3}$-valued 1 -form $\omega$ on $\mathcal{F}$, called the tautological 1-form, is defined by letting $\omega(v)=u\left(\pi_{*}(v)\right)$ for $v \in T_{u} \mathcal{F}$. The 1 -form $\omega$ has the $\operatorname{GL}\left(\mathcal{V}_{3}\right)$-equivariance $R_{g}{ }^{*}(\omega)=g^{-1} \omega$.

An $H_{3}$-structure on $M$ is, by definition, an $H_{3}$-subbundle $F \subset \mathcal{F}$. Note that the set of such $H_{3}$-structures on $M$ is in one-to-one correspondence with the set of sections of the quotient bundle $\bar{\pi}: \mathcal{F} / H_{3} \rightarrow M$ whose general fiber is isomorphic to $\operatorname{GL}\left(\mathcal{V}_{3}\right) / H_{3}$, a homogeneous space of dimension 13 . For any $H_{3}$-structure $F$, we will denote the restriction of $\pi$ and $\omega$ to $F$ by the same letters.

We shall first show that an $H_{3}$-structure $F$ has a canonical connection. Since $H_{3}$ is canonically isomorphic to $\mathrm{SL}(2, \mathbb{R})$, we may regard the $\mathrm{SL}(2, \mathbb{R})$-representations $\mathcal{V}_{d}$ equally well as $H_{3}$-representations. Moreover, since $\mathfrak{s l}(2, \mathbb{R}) \simeq \mathcal{V}_{2}$, it is easily seen that the map $\rho_{3}^{\prime}: \mathcal{V}_{2} \rightarrow \operatorname{End}\left(\mathcal{V}_{3}\right)$ defined, for $a \in \mathcal{V}_{2}$, by $\rho_{3}(a)(b)=\langle a, b\rangle_{1}$ establishes an isomorphism $\mathfrak{h}_{3} \simeq$ $\mathcal{V}_{2}$. We will use this to regard a connection on $F$ as an $H_{3}$-equivariant, $\mathcal{V}_{2}$-valued 1 -form $\phi$ on $F$. The torsion of $\phi$ is then represented by the $\mathcal{V}_{3}$-valued 2 -form $T(\phi)=d \omega+\langle\phi, \omega\rangle_{1}$ and the curvature of $\phi$ is represented by the $\mathcal{V}_{2}$-valued 2 -form $R(\phi)=d \phi+\frac{1}{2}\langle\phi, \phi\rangle_{1}$.

By Clebsch-Gordan, $\operatorname{hom}\left(\mathcal{V}_{3}, \mathcal{V}_{2}\right) \simeq \mathcal{V}_{1} \oplus \mathcal{V}_{3} \oplus \mathcal{V}_{5}$, so once one connection $\phi_{0}$ has been chosen, any other connection $\phi$ can be written uniquely in the form

$$
\phi=\phi_{0}+\left\langle p^{1}, \omega\right\rangle_{1}+\left\langle p^{3}, \omega\right\rangle_{2}+\left\langle p^{5}, \omega\right\rangle_{3},
$$


where each $p^{i}$ is an $H_{3}$-equivariant, $\mathcal{V}_{i}$-valued function on $F$. Using appropriate identities on the pairings $\langle,\rangle_{i}$ we then have

$$
\begin{aligned}
T(\phi) & =T\left(\phi_{0}\right)+\left\langle\left\langle p^{1}, \omega\right\rangle_{1}+\left\langle p^{3}, \omega\right\rangle_{2}+\left\langle p^{5}, \omega\right\rangle_{3}, \omega\right\rangle_{1} \\
& =T\left(\phi_{0}\right)+\frac{1}{2}\left\langle p^{1},\langle\omega, \omega\rangle_{1}\right\rangle_{1}+\frac{15}{4} p^{3}\langle\omega, \omega\rangle_{3}+\frac{1}{12}\left\langle p^{3},\langle\omega, \omega\rangle_{1}\right\rangle_{2}-\frac{1}{6}\left\langle p^{5},\langle\omega, \omega\rangle_{1}\right\rangle_{3} .
\end{aligned}
$$

Now, $T\left(\phi_{0}\right)$ can be written uniquely in the form

$$
T\left(\phi_{0}\right)=\left\langle t^{1},\langle\omega, \omega\rangle_{1}\right\rangle_{1}+s^{3}\langle\omega, \omega\rangle_{3}+\left\langle t^{3},\langle\omega, \omega\rangle_{1}\right\rangle_{2}+\left\langle t^{5},\langle\omega, \omega\rangle_{1}\right\rangle_{3}+\left\langle t^{7},\langle\omega, \omega\rangle_{1}\right\rangle_{4}
$$

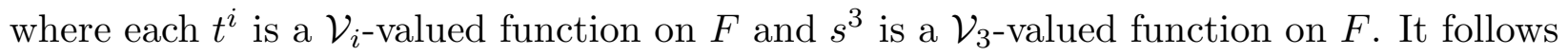
that there is a unique connection $\phi$ on $F$ for which

$$
T(\phi)=\left\langle\tau^{3},\langle\omega, \omega\rangle_{1}\right\rangle_{2}+\left\langle\tau^{7},\langle\omega, \omega\rangle_{1}\right\rangle_{4}
$$

for some $\mathcal{V}_{3}$-valued function $\tau^{3}$ and some $\mathcal{V}_{7}$-valued function $\tau^{7}$ on $F$.

We call this $\phi$ the intrinsic connection of $F$ and we call the resulting torsion the intrinsic torsion of $F$. We shall say that $F$ is 1-flat or torsion-free if its intrinsic torsion vanishes. (For an explanation of the term "1-flat," see Bryant [1987]).

The equations $\tau^{3}=\tau^{7}=0$ may be regarded as a set of 12 first order PDE for the section of the bundle $\mathcal{F} / H_{3}$ which determines $F$. Thus, locally, these represent 12 first order equations for 13 unknowns. However, because this "underdetermined" system is invariant under the diffeomorphism group of $M$, its behavior is hard to understand directly via the classical approaches to PDE systems. In particular, proving local existence of any local solutions other than the flat solution is non-trivial and will be taken up in $\S 3$.

There is a natural equivalence relation, homothety, on $\mathrm{H}_{3}$-structures which is defined as follows. Regard $\mathbb{R}^{+}$, the positive real numbers, as a subgroup of $\operatorname{GL}\left(\mathcal{V}_{3}\right)$ by regarding $t \in \mathbb{R}^{+}$as $t$ times the identity mapping. Then, on $\mathcal{F}, R_{t}{ }^{*}(\omega)=t^{-1} \omega$. For any $H_{3}$-structure $F$, we can define $F^{t}=R_{t}(F)$. We say that $F^{t}$ and $F$ are homothetic and the set of $H_{3^{-}}$ structures homothetic to $F$ will be called its homothety class. The mapping $R_{t}: F \rightarrow F^{t}$ is a bundle mapping and satisfies $R_{t}{ }^{*}\left(\omega^{t}\right)=t^{-1} \omega$ and $R_{t}{ }^{*}\left(\phi^{t}\right)=\phi$ where $\omega^{t}$ and $\phi^{t}$ are the tautological form and intrinsic connection, respectively, of $F^{t}$. It follows that $R_{t}{ }^{*}\left(\left(\tau^{3}\right)^{t}\right)=t \tau^{3}$ and $R_{t}{ }^{*}\left(\left(\tau^{7}\right)^{t}\right)=t \tau^{7}$. In particular, the condition of being torsion-free is a homothety invariant.

Henceforth, $F$ will denote a torsion-free $H_{3}$-structure on $M$ and $\phi$ will denote its intrinsic connection. We thus have the first structure equation:

$$
d \omega=-\langle\phi, \omega\rangle_{1}
$$

Differentiating this equation then yields the relation $\langle R(\phi), \omega\rangle_{1}=0$, which is the first Bianchi identity. According to Table I of $\S 1$, the curvature of $\phi$ must be represented by a $\mathcal{V}_{2}$-valued function on $F$. In fact, solving the first Bianchi identity shows that there is 
a unique $\mathcal{V}_{2}$-valued function $a$ on $F$ for which the following relation, known as the second structure equation, holds:

$$
\begin{aligned}
d \phi & =-\frac{1}{2}\langle\phi, \phi\rangle_{1}+R(\phi) \\
& =-\frac{1}{2}\langle\phi, \phi\rangle_{1}+a\langle\omega, \omega\rangle_{3}-\frac{1}{12}\left\langle a,\langle\omega, \omega\rangle_{1}\right\rangle_{2} .
\end{aligned}
$$

The following proposition explains the relationship of torsion-free $H_{3}$-structures on $M$ with torsion-free connections on $M$ whose holonomy is conjugate to $\mathrm{H}_{3}$.

Proposition 2.1: Let $M$ be a smooth, simply connected 4-manifold. There is a one-to-one correspondence between the set of torsion-free affine connections on $M$ whose holonomy is conjugate to a non-trivial subgroup of $\mathrm{H}_{3}$ and the homothety classes of torsion-free $H_{3}$-structures on $\mathrm{M}$ whose canonical connections are not flat.

ProOF: Let $F$ be a torsion-free $H_{3}$-structure on $M$ whose canonical connection $\phi$ is not flat. Let $\psi$ denote the $\mathfrak{g l}\left(\mathcal{V}_{3}\right)$-valued connection 1 -form on $\mathcal{F}$ which restricts to $F$ to become $\phi$. Then $\psi$ is a torsion-free connection on $\mathcal{F}$ and hence corresponds to a unique torsion-free affine connection $\nabla_{F}$ on $M$. By construction, $F$ is invariant under $\nabla_{F}$-parallel transport and it follows that the holonomy of $\nabla_{F}$ is conjugate to a subgroup of $H_{3}$. Note that $\nabla_{F^{t}}=\nabla_{F}$, so $\nabla_{F}$ depends only on the homothety class of $F$.

Conversely, let $\nabla$ be a torsion-free affine connection on $M$ whose holonomy is conjugate to a non-trivial subgroup of $H_{3}$. Since the holonomy of $\nabla$ is non-trivial, by the AmbroseSinger Holonomy Theorem, there must be a point $x \in M$ at which the curvature $R^{\nabla}$ is not zero. Let $u \in \mathcal{F}_{x}$ be a linear isomorphism $u: T_{\pi(u)} M \stackrel{\sim}{\rightarrow} \mathcal{V}_{3}$ which induces an isomorphism $H_{x} \stackrel{\sim}{\rightarrow} H \subset H_{3}$, where $H$ is a non-trivial connected Lie subgroup of $H_{3}$. Let $P(u) \subset \mathcal{F}$ denote the $\nabla$-holonomy bundle through $u$, and let $P_{+}(u)=P(u) \cdot H_{3}$ denote its extension to an $H_{3}$-subbundle of $\mathcal{F}$. Let $\psi$ denote the $\mathfrak{g l}\left(\mathcal{V}_{3}\right)$-valued 1 -form on $\mathcal{F}$ which corresponds to $\nabla$.

Because the $H_{3}$-structure $P_{+}(u)$ contains $P(u)$, it follows that $P_{+}(u)$ is preserved by $\nabla$-parallel transport and hence that $\psi$ restricts to $P_{+}(u)$ to become a 1 -form $\phi$ with values in $\mathfrak{h}_{3} \simeq \mathcal{V}_{2}$ and which is a connection on $P_{+}(u)$. Since $\psi$ is torsion-free, it follows that $\phi$ is also. It follows that $\phi$ is the intrinsic connection of $F=P_{+}(u)$ and that $F$ is torsion-free. In particular, (1) and (2) hold.

If, on $F$, we write

$$
\omega=\omega_{-3} x^{3}+\omega_{-1} x^{2} y+\omega_{1} x y^{2}+\omega_{3} y^{3}
$$

and

$$
a=a_{-2} x^{2}+a_{0} x y+a_{2} y^{2}
$$

then the formula for $R(\phi)$ can be expressed as

$$
\begin{aligned}
R(\phi)= & \left(a_{-2}\left(9 \omega_{-3} \wedge \omega_{3}-5 \omega_{-1} \wedge \omega_{1}\right)+3 a_{0} \omega_{-3} \wedge \omega_{1}-6 a_{2} \omega_{-3} \wedge \omega_{-1}\right) x^{2} \\
+ & \left(-6 a_{-2} \omega_{-1} \wedge \omega_{3}+a_{0}\left(18 \omega_{-3} \wedge \omega_{3}-2 \omega_{-1} \wedge \omega_{1}\right)-6 a_{2} \omega_{-3} \wedge \omega_{1}\right) x y \\
+ & \left(-6 a_{-2} \omega_{1} \wedge \omega_{3}+3 a_{0} \omega_{-1} \wedge \omega_{3}+a_{2}\left(9 \omega_{-3} \wedge \omega_{3}-5 \omega_{-1} \wedge \omega_{1}\right)\right) y^{2}
\end{aligned}
$$


Since $R^{\nabla}$ is non-zero at $u \in F$, it follows that $a(u)$ is non-zero. From this explicit formula for $R(\phi)$ it follows that, at $u$, this 2 -form does not have values in any proper subspace of $\mathcal{V}_{2}$ and hence that the holonomy algebra $\mathfrak{h}$ cannot be a proper subspace of $\mathfrak{h}_{3}$. In particular, again by an application of the Ambrose-Singer Holonomy Theorem, we must have $H=H_{3}$ and $F=P_{+}(u)=P(u)$.

Now let $u^{\prime} \in \mathcal{F}$ be any other coframe $u^{\prime}: T_{\pi\left(u^{\prime}\right)} M \stackrel{\sim}{\rightarrow} \mathcal{V}_{3}$ which induces an isomorphism $\mathcal{H}_{\pi\left(u^{\prime}\right)}(\nabla) \stackrel{\sim}{\rightarrow} H_{3}$. By parallel transport, we may assume that $\pi\left(u^{\prime}\right)=\pi(u)=x$. It follows that $u^{\prime}=A \circ u$ where $A$ is an element of the normalizer of $H_{3}$ in $\operatorname{GL}\left(\mathcal{V}_{3}\right)$. However, it is easily seen that this normalizer is $G_{3}$. In particular, $A=t g$ where $g \in H_{3}$ and $t \in \mathbb{R}^{+}$. It follows that $P\left(u^{\prime}\right)=R_{t}(P(u))=F^{t}$. Thus, $\nabla$ canonically determines the homothety class of $F$.

The monicity of the correspondence just constructed is now immediate.

It is worth remarking that, in the course of the above proof, we showed that no nontrivial proper subgroup of $H_{3}$ can be the holonomy of an affine torsion-free connection on $M$.

Let us now explore the consequences of the structure equations (1) and (2). If we write $D a=d a+\langle\phi, a\rangle_{1}$, then the derivative of (2) becomes the relation $D a \wedge\langle\omega, \omega\rangle_{3}-$ $\frac{1}{12}\left\langle D a,\langle\omega, \omega\rangle_{1}\right\rangle_{2}=0$. This relation can be solved, showing that $D a=\langle b, \omega\rangle_{2}$ for some unique $\mathcal{V}_{3}$-valued function $b$ on $F$. This gives the third structure equation,

$$
d a=-\langle\phi, a\rangle_{1}+\langle b, \omega\rangle_{2} .
$$

Setting $D b=d b+\langle\phi, b\rangle_{1}$ and differentiating (3), gives the relation $-\langle R(\phi), a\rangle_{1}+\langle D b, \omega\rangle_{2}=$ 0 , which can be solved for $D b$ to show that there exists a $\mathcal{V}_{0}$-valued function $c$ on $F$ so that the fourth structure equation holds:

$$
d b=-\langle\phi, b\rangle_{1}+\left(c-\langle a, a\rangle_{2}\right) \omega+\frac{1}{12}\left\langle\langle a, a\rangle_{0}, \omega\right\rangle_{2} .
$$

Finally, differentiation of (4) gives the fifth structure equation

$$
d c=0 \text {. }
$$

No further relations can be deduced by differentiation of the equations (1)-(5). In the next section, we will use these equations to establish the existence of non-flat, torsion-free $\mathrm{H}_{3}$-structures and determine their "generality."

We now turn to the case of $G_{3} \subset \mathrm{GL}\left(\mathcal{V}_{3}\right)$ which, at first, is similar to that of $H_{3}$. Let $F \subset \mathcal{F}$ be an $G_{3}$ subbundle, i.e., a $G_{3}$-structure on $M$. We denote the restriction of $\pi$ and $\omega$ to $F$ by the same letters. Note that the set of $G_{3}$-structures on $M$ is in one-to-one correspondence with the set of sections of the quotient bundle $\bar{\pi}: \mathcal{F} / G_{3} \rightarrow M$ whose general fiber is isomorphic to $\operatorname{GL}\left(\mathcal{V}_{3}\right) / G_{3}$, a homogeneous space of dimension 12 .

Again, we wish to introduce a canonical connection on $F$. Now $\mathfrak{g}_{3}=\mathbb{R} \cdot \mathrm{id} \mathcal{V}_{3}+\mathfrak{h}_{3} \simeq$ $\mathcal{V}_{0} \oplus \mathcal{V}_{2}$. We will use these identifications to regard a connection on $F$ as an equivariant 
$\left(\mathcal{V}_{0} \oplus \mathcal{V}_{2}\right)$-valued 1 -form $\varphi=\lambda+\phi$ on $F$, where $\lambda$ is a $\mathcal{V}_{0}$-valued 1 -form on $F$ and $\phi$ is, as before, a $\mathcal{V}_{2}$-valued 1 -form on $F$. The torsion of $\varphi$ is represented by the $\mathcal{V}_{3}$-valued 2 -form $T(\varphi)=d \omega+\lambda \wedge \omega+\langle\phi, \omega\rangle_{1}$ and the curvature of $\varphi$ is represented by the $\left(\mathcal{V}_{0} \oplus \mathcal{V}_{2}\right)$-valued 2 -form $R(\varphi)=d \varphi+\frac{1}{2}\langle\varphi, \varphi\rangle_{1}=d \lambda+d \phi+\frac{1}{2}\langle\phi, \phi\rangle_{1}$.

By an argument entirely analogous to the one for $H_{3}$ and which need not be recounted here, it can be shown that there is a unique connection $\varphi$ on $F$ for which the torsion takes the form

$$
T(\varphi)=\left\langle\tau,\langle\omega, \omega\rangle_{1}\right\rangle_{4}
$$

for some some $\mathcal{V}_{7}$-valued function $\tau$ on $F$. We call the resulting connection $\varphi$ the intrinsic connection of $F$ and the resulting torsion the intrinsic torsion of $F$. We say that $F$ is 1-flat or torsion-free if its intrinsic torsion vanishes.

The equations $\tau=0$ represent a set of 8 first order PDE for the section of the bundle $\mathcal{F} / G_{3}$ which determines $F$. Thus, locally, these represent 8 first order equations for 12 unknowns. As in the $H_{3}$ case, because these equations are invariant under the diffeomorphism group of $M$, they cannot be written locally as an "underdetermined Cauchy-Kowalewski system," in the usual sense. Thus, a direct application of PDE techniques to the study of the "generality" of the solutions of these equations modulo diffeomorphism equivalence is not easy. It is not at all obvious that this "solution space" is even non-empty.

For the remainder of this section, $F$ will denote a torsion-free $G_{3}$-structure on $M$ and $\varphi$ will denote its intrinsic connection. The first structure equation is then

$$
d \omega=-\lambda \wedge \omega-\langle\phi, \omega\rangle_{1}
$$

Differentiating (6) then yields the relation

$$
d \lambda \wedge \omega+\left\langle d \phi+\frac{1}{2}\langle\phi, \phi\rangle_{1}, \omega\right\rangle_{1}=0
$$

which is the first Bianchi identity. According to Table II of $\S 1$, the curvature of $\varphi$ must be represented by a $\left(\mathcal{V}_{2} \oplus \mathcal{V}_{4}\right)$-valued function on $F$. In fact, solving the first Bianchi identity shows that there exist on $F$ a unique $\mathcal{V}_{2}$-valued function $a^{2}$ and a unique $\mathcal{V}_{4}$-valued function $a^{4}$ for which the following relations, the second structure equations, hold:

$$
\begin{aligned}
& d \lambda=\left\langle a^{4},\langle\omega, \omega\rangle_{1}\right\rangle_{4} \\
& d \phi=-\frac{1}{2}\langle\phi, \phi\rangle_{1}+a^{2}\langle\omega, \omega\rangle_{3}-\frac{1}{12}\left\langle a^{2},\langle\omega, \omega\rangle_{1}\right\rangle_{2}+\frac{1}{12}\left\langle a^{4},\langle\omega, \omega\rangle_{1}\right\rangle_{3} .
\end{aligned}
$$

Unfortunately, there does not seem to be a simple analogue of Proposition 2.1 for the case of $G_{3}$. This is due, in part, to the fact that $\mathfrak{g}_{3}$ contains non-trivial algebras $\mathfrak{h}$ (besides $\mathfrak{h}_{3}$ ) for which $\mathcal{K}(\mathfrak{h}) \neq 0$. However, we do have the following statement.

Proposition 2.2: Let $M$ be a smooth, simply connected 4-manifold. Any torsion-free $G_{3^{-}}$ structure $F$ on $M$ determines a unique torsion-free affine connection on $M$ whose holonomy is (conjugate to) a subgroup of $G_{3}$. Conversely, any torsion-free affine connection on $M$ whose holonomy is (conjugate to) $G_{3}$ corresponds to a unique torsion-free $G_{3}$-structure on $M$. 
PROOF: The construction of the correspondence and proof of this proposition are completely analogous to those of Proposition 2.1. The essential point in the proof of the second statement of the proposition is that $G_{3}$ is its own normalizer in $\operatorname{GL}\left(\mathcal{V}_{3}\right)$.

Finally, for use in the next section, we derive the $G_{3}$-analogues of the third and fourth structure equations. A glance at Table II of $\S 1$ shows that we should expect the first covariant derivative of the curvature tensor of $\varphi$ to be represented by a function on $F$ which takes values in the vector space $\mathcal{V}_{1} \oplus \mathcal{V}_{3} \oplus \mathcal{V}_{5} \oplus \mathcal{V}_{7}$. In fact, computing the exterior derivative of (7) and solving the resulting relations allows us to show that, for each $i=1$, 3,5 , or 7 , there exists a unique $\mathcal{V}_{i}$-function $b^{i}$ on $F$ so that the following equations hold:

$$
\begin{aligned}
& d a^{2}=2 \lambda \wedge a^{2}-\left\langle\phi, a^{2}\right\rangle_{1}+10\left\langle b^{1}, \omega\right\rangle_{1}+\left\langle b^{3}, \omega\right\rangle_{2}+14\left\langle b^{5}, \omega\right\rangle_{3} \\
& d a^{4}=2 \lambda \wedge a^{4}-\left\langle\phi, a^{4}\right\rangle_{1}+9\left\langle b^{1}, \omega\right\rangle_{0}-5\left\langle b^{5}, \omega\right\rangle_{2}+\left\langle b^{7}, \omega\right\rangle_{3}
\end{aligned}
$$

We shall need formulas for the derivatives of the functions $b^{i}$ as well. This leads to a rather formidable linear algebra problem. Fortunately, however, this can be solved easily by simple MAPLE procedures. We merely record the result here: Set

$$
\begin{aligned}
\beta^{1}= & d b^{1}-3 \lambda \wedge b^{1}+\left\langle\phi, b^{1}\right\rangle_{1} \\
& +\frac{1}{30}\left\langle\left\langle a^{2}, a^{4}\right\rangle_{2}, \omega\right\rangle_{2}-\left\langle\frac{1}{108}\left\langle a^{4}, a^{4}\right\rangle_{2}-\frac{1}{16}\left\langle a^{2}, a^{4}\right\rangle_{1}, \omega\right\rangle_{3} \\
\beta^{3}= & d b^{3}-3 \lambda \wedge b^{3}+\left\langle\phi, b^{3}\right\rangle_{1}+\frac{1}{15}\left\langle\left\langle a^{2}, a^{4}\right\rangle_{2}, \omega\right\rangle_{1} \\
& -\left\langle\frac{1}{405}\left\langle a^{4}, a^{4}\right\rangle_{2}-\frac{1}{30}\left\langle a^{2}, a^{4}\right\rangle_{1}+\frac{1}{12}\left\langle a^{2}, a^{2}\right\rangle_{0}, \omega\right\rangle_{2}-\frac{4}{25}\left\langle\left\langle a^{2}, a^{4}\right\rangle_{0}, \omega\right\rangle_{3} \\
\beta^{5}= & d b^{5}-3 \lambda \wedge b^{5}+\left\langle\phi, b^{5}\right\rangle_{1}-\left\langle\frac{1}{1260}\left\langle a^{4}, a^{4}\right\rangle_{2}+\frac{1}{240}\left\langle a^{2}, a^{4}\right\rangle_{1}, \omega\right\rangle_{1} \\
& +\frac{1}{150}\left\langle\left\langle a^{2}, a^{4}\right\rangle_{0}, \omega\right\rangle_{2}+\frac{1}{70}\left\langle\left\langle a^{4}, a^{4}\right\rangle_{0}, \omega\right\rangle_{3} \\
\beta^{7}= & d b^{7}-3 \lambda \wedge b^{7}+\left\langle\phi, b^{7}\right\rangle_{1} .
\end{aligned}
$$

A calculation then shows that the exterior derivatives of the equations (8) can be written in the form

$$
\begin{aligned}
& 0=10\left\langle\beta^{1}, \omega\right\rangle_{1}+\left\langle\beta^{3}, \omega\right\rangle_{2}+14\left\langle\beta^{5}, \omega\right\rangle_{3} \\
& 0=9\left\langle\beta^{1}, \omega\right\rangle_{0} \quad-5\left\langle\beta^{5}, \omega\right\rangle_{2}+\left\langle\beta^{7}, \omega\right\rangle_{3} .
\end{aligned}
$$

Another calculation then shows that, for each $i=0,2,4,6,8$, or, 10 there exists a unique $\mathcal{V}_{i}$-valued function $c^{i}$ on $F$ so that

$$
\begin{aligned}
& \beta^{1}=\quad-35\left\langle c^{2}, \omega\right\rangle_{2}-147\left\langle c^{4}, \omega\right\rangle_{3} \\
& \beta^{3}=\quad\left\langle c^{0}, \omega\right\rangle_{0}+56\left\langle c^{2}, \omega\right\rangle_{1}-392\left\langle c^{4}, \omega\right\rangle_{2}+168\left\langle c^{6}, \omega\right\rangle_{3} \\
& \beta^{5}=9\left\langle c^{2}, \omega\right\rangle_{0}+9\left\langle c^{4}, \omega\right\rangle_{1}+3\left\langle c^{6}, \omega\right\rangle_{2}+\left\langle c^{8}, \omega\right\rangle_{3} \\
& \beta^{7}=-162\left\langle c^{4}, \omega\right\rangle_{0}+10\left\langle c^{6}, \omega\right\rangle_{1}-2\left\langle c^{8}, \omega\right\rangle_{2}+\left\langle c^{10}, \omega\right\rangle_{3} .
\end{aligned}
$$

The main importance of this formula (as we shall see in the next section) is that the space of solutions of the equations (10) at each point of $F$ has dimension

$$
\operatorname{dim}\left(\mathcal{V}_{0} \oplus \mathcal{V}_{2} \oplus \mathcal{V}_{4} \oplus \mathcal{V}_{6} \oplus \mathcal{V}_{8} \oplus \mathcal{V}_{10}\right)=36
$$




\section{§3. The Existence Theorems}

In this section, we turn to the question of the existence and "generality" of the set of torsion-free $\mathrm{H}_{3}$ - or $G_{3}$-structures on 4-manifolds. These problems, though expressible locally in terms of PDE, are rather difficult to treat directly because of their diffeomorphism invariance. Our approach will be to cast these problems as problems in exterior differential systems via a technique due originally to Élie Cartan [1943].

We will begin by treating the case of $G_{3}$. First, we note that we can just as well regard $\mathcal{V}_{p}$ for each $p \geq 0$ as a $G L^{+}(2, \mathbb{R})$ representation where $G L^{+}(2, \mathbb{R})$ is the group of linear transformations in the two variables $x$ and $y$ with positive determinant. However, note that the pairings $\langle,\rangle_{p}$ are not $G L^{+}(2, \mathbb{R})$-equivariant for $p>0$. For the sake of simplicity, we shall denote the action of $g \in G L^{+}(2, \mathbb{R})$ on $v \in \mathcal{V}_{p}$ by $g \cdot v$. Note that in the case $p=3$ this establishes a canonical isomorphism $G_{3}=G L^{+}(2, \mathbb{R})$. We shall identify these two groups via this isomorphism from now on when there is no possibility of confusion.

Suppose that $F$ is a torsion-free $G_{3}$-structure on a 4 -manifold $M$. The structure equations derived in the last section then show that we have $G_{3}$-equivariant mappings $a^{i}: F \rightarrow \mathcal{V}_{i}$ for $i=2$ or 4 , and $b^{j}: F \rightarrow \mathcal{V}_{j}$ for $j=1,3,5$ or 7 . To simplify our notation, let us set $\mathcal{V}=\mathcal{V}_{2} \oplus \mathcal{V}_{4}$ and $\mathcal{W}=\mathcal{V}_{1} \oplus \mathcal{V}_{3} \oplus \mathcal{V}_{5} \oplus \mathcal{V}_{7}$ and let $a=a^{2}+a^{4}$ and $b=b^{1}+b^{3}+b^{5}+b^{7}$ be regarded as mappings of $F$ into $\mathcal{V}$ and $\mathcal{W}$ respectively. We define the total curvature mapping of $F$ to be the map $K: F \rightarrow \mathcal{V} \oplus \mathcal{W}$ where $K=a+b$.

We shall say that $F$ is non-degenerate if the mapping $a: F \rightarrow \mathcal{V}$ is a local diffeomorphism. Since $F$ and $\mathcal{V}$ both have dimension 8, this is not an unreasonable notion. Indeed, if non-degenerate, torsion-free $G_{3}$-structures exist, one expects them, in some sense, to be "generic" among torsion-free $G_{3}$-structures. If $F$ is non-degenerate, the mapping $K: F \rightarrow \mathcal{V} \oplus \mathcal{W}$ determines an 8-dimensional, $G_{3}$-invariant, immersed submanifold of $\mathcal{V} \oplus \mathcal{W}$.

We shall now show how this process can be reversed, at least locally. That is, we are going to show that the image $K(F)$ determines $M$ and $F$ locally up to diffeomorphism. Moreover, we shall show that $K(F)$ is characterized as an integral manifold of a certain exterior differential system with independence condition.

We now turn to the construction of this differential system. Let $\mathbf{a}^{i}: \mathcal{V} \oplus \mathcal{W} \rightarrow \mathcal{V}_{i}$ for $i=2$ or 4 , and $\mathbf{b}^{j}: \mathcal{V} \oplus \mathcal{W} \rightarrow \mathcal{V}_{j}$ for $j=1,3,5$ or 7 denote the projections thought of as vector-valued functions on $\mathcal{V} \oplus \mathcal{W}$. We would like to define 1-forms $\lambda$, $\boldsymbol{\phi}$, and $\boldsymbol{\omega}$ on $\mathcal{V} \oplus \mathcal{W}$ with values in $\mathcal{V}_{0}, \mathcal{V}_{2}$, and $\mathcal{V}_{3}$ respectively, so that the following equations hold:

$$
\begin{aligned}
& d \mathbf{a}^{2}=2 \boldsymbol{\lambda} \wedge \mathbf{a}^{2}-\left\langle\boldsymbol{\phi}, \mathbf{a}^{2}\right\rangle_{1}+10\left\langle\mathbf{b}^{1}, \boldsymbol{\omega}\right\rangle_{1}+\left\langle\mathbf{b}^{3}, \boldsymbol{\omega}\right\rangle_{2}+14\left\langle\mathbf{b}^{5}, \boldsymbol{\omega}\right\rangle_{3} \\
& d \mathbf{a}^{4}=2 \boldsymbol{\lambda} \wedge \mathbf{a}^{4}-\left\langle\boldsymbol{\phi}, \mathbf{a}^{4}\right\rangle_{1}+9\left\langle\mathbf{b}^{1}, \boldsymbol{\omega}\right\rangle_{0}-5\left\langle\mathbf{b}^{5}, \boldsymbol{\omega}\right\rangle_{2}+\left\langle\mathbf{b}^{7}, \boldsymbol{\omega}\right\rangle_{3} .
\end{aligned}
$$

Setting $\mathbf{a}=\mathbf{a}^{2}+\mathbf{a}^{4}$ and $\mathbf{b}=\mathbf{b}^{1}+\mathbf{b}^{3}+\mathbf{b}^{5}+\mathbf{b}^{7}$, this set of equations can be written in the form

$$
d \mathbf{a}=\mathbf{A}(\mathbf{a})(\boldsymbol{\lambda}+\boldsymbol{\phi})+\mathbf{B}(\mathbf{b})(\boldsymbol{\omega})
$$


where $\mathbf{A}$ is linear mapping from $\mathcal{V}$ to $\operatorname{hom}\left(\mathcal{V}_{0} \oplus \mathcal{V}_{2}, \mathcal{V}_{2} \oplus \mathcal{V}_{4}\right)$ and $\mathbf{B}$ is linear mapping from $\mathcal{W}$ to $\operatorname{hom}\left(\mathcal{V}_{3}, \mathcal{V}_{2} \oplus \mathcal{V}_{4}\right)$. Regarding $\mathbf{A}(\mathbf{a})$ and $\mathbf{B}(\mathbf{b})$ as vector-valued functions on $\mathcal{V} \oplus \mathcal{W}$, we can define a function $\mathbf{J}$ on $\mathcal{V} \oplus \mathcal{W}$ with values in $\operatorname{hom}\left(\mathcal{V}_{0} \oplus \mathcal{V}_{2} \oplus \mathcal{V}_{3}, \mathcal{V}_{2} \oplus \mathcal{V}_{4}\right)$ by the property that $\mathbf{J}(u+v)=\mathbf{A}(\mathbf{a})(u)+\mathbf{B}(\mathbf{b})(v)$ for all $u \in \mathcal{V}_{0} \oplus \mathcal{V}_{2}$ and $v \in \mathcal{V}_{3}$. Thus, the above equation can also be written as $d \mathbf{a}=\mathbf{J}(\boldsymbol{\lambda}+\boldsymbol{\phi}+\boldsymbol{\omega})$. Since the vector spaces $\mathcal{V}_{0} \oplus \mathcal{V}_{2} \oplus \mathcal{V}_{3}$ and $\mathcal{V}_{2} \oplus \mathcal{V}_{4}$ both have dimension 8, it follows that, relative to the standard bases of these two spaces, $\mathbf{J}$ has a representation as a square matrix (whose entries are linear functions on $\mathcal{V} \oplus \mathcal{W}$ ). Let $\mathbf{D}$ be the determinant of this matrix representation of $\mathbf{J}$. Then $\mathbf{D}$ is a polynomial function of degree 8 on $\mathcal{V} \oplus \mathcal{W}$ and it is not hard to check (using MAPLE) that $\mathbf{D}$ does not vanish identically.

Let $\mathcal{O} \subset \mathcal{V} \oplus \mathcal{W}$ denote the open set where $\mathbf{D}$ is non-zero. Then, on $\mathcal{O}$, we define $\boldsymbol{\lambda}$, $\boldsymbol{\phi}$, and $\boldsymbol{\omega}$ by the formula

$$
\lambda+\boldsymbol{\phi}+\boldsymbol{\omega}=\mathbf{J}^{-1} d \mathbf{a} .
$$

Note that, with this definition, the equations (1) become identities. Moreover, if we set

$$
\Omega=\lambda_{0} \wedge \boldsymbol{\phi}_{-2} \wedge \boldsymbol{\phi}_{0} \wedge \boldsymbol{\phi}_{2} \wedge \boldsymbol{\omega}_{-3} \wedge \boldsymbol{\omega}_{-1} \wedge \boldsymbol{\omega}_{1} \wedge \boldsymbol{\omega}_{3}
$$

then we have

$$
\Omega=\mathbf{D}^{-1} d \mathbf{a}_{-2}^{2} \wedge d \mathbf{a}_{0}^{2} \wedge d \mathbf{a}_{2}^{2} \wedge d \mathbf{a}_{-4}^{4} \wedge d \mathbf{a}_{-2}^{4} \wedge d \mathbf{a}_{0}^{4} \wedge d \mathbf{a}_{2}^{4} \wedge d \mathbf{a}_{4}^{4} .
$$

We are now ready to define an exterior differential system on $\mathcal{O}$. First, we define the following 2-forms on $\mathcal{O}$ :

$$
\begin{aligned}
& \boldsymbol{\Theta}=d \boldsymbol{\omega}+\boldsymbol{\lambda} \wedge \boldsymbol{\omega}+\langle\boldsymbol{\phi}, \boldsymbol{\omega}\rangle_{1}, \\
& \boldsymbol{\Lambda}=d \boldsymbol{\lambda}-\left\langle\mathbf{a}^{4},\langle\boldsymbol{\omega}, \boldsymbol{\omega}\rangle_{1}\right\rangle_{4} \\
& \boldsymbol{\Phi}=d \boldsymbol{\Phi}+\frac{1}{2}\langle\boldsymbol{\phi}, \boldsymbol{\phi}\rangle_{1}-\mathbf{a}^{2}\langle\boldsymbol{\omega}, \boldsymbol{\omega}\rangle_{3}+\frac{1}{12}\left\langle\mathbf{a}^{2},\langle\boldsymbol{\omega}, \boldsymbol{\omega}\rangle_{1}\right\rangle_{2}-\frac{1}{12}\left\langle\mathbf{a}^{4},\langle\boldsymbol{\omega}, \boldsymbol{\omega}\rangle_{1}\right\rangle_{3} .
\end{aligned}
$$

Using the identities (1), it is straightforward to compute that the exterior derivatives of these 2 -forms are given by the formulas

$$
\begin{aligned}
d \boldsymbol{\Theta} & =\boldsymbol{\Lambda} \wedge \boldsymbol{\omega}-\boldsymbol{\lambda} \wedge \boldsymbol{\Theta}+\langle\boldsymbol{\Phi}, \boldsymbol{\omega}\rangle_{1}-\langle\boldsymbol{\Phi}, \boldsymbol{\Theta}\rangle_{1}, \\
d \boldsymbol{\Lambda} & =2\left\langle\mathbf{a}^{4},\langle\boldsymbol{\omega}, \boldsymbol{\Theta}\rangle_{1}\right\rangle_{4} \\
d \boldsymbol{\Phi} & =-\langle\boldsymbol{\Phi}, \boldsymbol{\Phi}\rangle_{1}+2 \mathbf{a}^{2}\langle\boldsymbol{\omega}, \boldsymbol{\Theta}\rangle_{3}-\frac{1}{6}\left\langle\mathbf{a}^{2},\langle\boldsymbol{\omega}, \boldsymbol{\Theta}\rangle_{1}\right\rangle_{2}+\frac{1}{6}\left\langle\mathbf{a}^{4},\langle\boldsymbol{\omega}, \boldsymbol{\Theta}\rangle_{1}\right\rangle_{3}
\end{aligned}
$$

It follows that the exterior ideal $\mathcal{I} \subset \Omega^{*}(\mathcal{O})$ generated by the eight 2 -forms

$$
\Lambda_{0}, \Phi_{-2}, \Phi_{0}, \Phi_{2}, \Theta_{-3}, \Theta_{-1}, \Theta_{1}, \Theta_{3}
$$

is differentially closed. 
Proposition 3.1: Let $F$ be a non-degenerate, torsion-free $G_{3}$-structure on a 4-manifold $M$. Then the curvature mapping $a+b: F \rightarrow \mathcal{V} \oplus \mathcal{W}$ has its image in $\mathcal{O} \subset \mathcal{V} \oplus \mathcal{W}$ and is an integral manifold of the differential system with independence condition $(\mathcal{I}, \Omega)$. Conversely, every integral manifold of $(\mathcal{I}, \Omega)$ is locally the image of a non-degenerate, torsion-free $G_{3^{-}}$ structure on some 4-manifold $M$ and this $G_{3}$-structure is unique up to diffeomorphism.

Proof: First, suppose that $F$ is a non-degenerate, torsion-free $G_{3}$-structure on $M$. Then the structure equations (2.6-8) hold for the canonical 1-forms $\omega, \lambda$, and $\phi$ and functions $a^{i}$ and $b^{j}$ on $F$. The curvature mapping $K=a+b$ satisfies $K: F \rightarrow \mathcal{V} \oplus \mathcal{W}$ and clearly satisfies $K^{*}\left(\mathbf{a}^{i}\right)=a^{i}$ and $K^{*}\left(\mathbf{b}^{j}\right)=b^{j}$. Now, (2.8) takes the form $d a=K^{*}(\mathbf{J})(\lambda+\phi+\omega)$. By the hypothesis that $F$ is non-degenerate, we know that the rank of the differential of the mapping $a: F \rightarrow \mathcal{V}$ is 8 , so it follows that $K^{*}(\mathbf{J})$ must be invertible. Thus, $K^{*}(\mathbf{D}) \neq 0$, so $K(F) \subset \mathcal{O}$. Now, pulling back the identity (2), we get

$$
\begin{aligned}
K^{*}(\boldsymbol{\lambda}+\boldsymbol{\phi}+\boldsymbol{\omega}) & =K^{*}(\mathbf{J})^{-1} d K^{*}(\mathbf{a}) \\
& =K^{*}(\mathbf{J})^{-1} d a=(\lambda+\phi+\omega) .
\end{aligned}
$$

Of course, it then follows that $K^{*}(\boldsymbol{\lambda})=\lambda, K^{*}(\boldsymbol{\phi})=\phi$, and $K^{*}(\boldsymbol{\omega})=\omega$. Then the structure equations $(2.6,7)$ imply that $K^{*}(\boldsymbol{\Lambda})=0, K^{*}(\boldsymbol{\Phi})=0$, and $K^{*}(\boldsymbol{\Theta})=0$. Moreover, we clearly have $K^{*}(\Omega) \neq 0$. Thus, $K: F \rightarrow \mathcal{V} \oplus \mathcal{W}$ is an integral manifold of $(\mathcal{I}, \Omega)$, as desired.

Now, for the converse. Let $P^{8} \subset \mathcal{O}$ be a connected integral manifold of $(\mathcal{I}, \Omega)$ and let $\iota: P \hookrightarrow \mathcal{O}$ denote the inclusion mapping. Let us define $\iota^{*}(\boldsymbol{\lambda})=\bar{\lambda}, \iota^{*}(\boldsymbol{\phi})=\bar{\phi}, \iota^{*}(\boldsymbol{\omega})=\bar{\omega}$, $\iota^{*}\left(\mathbf{a}^{i}\right)=\bar{a}^{i}$, and $\iota^{*}\left(\mathbf{b}^{j}\right)=\bar{b}^{j}$. Since $P$ is an integral manifold of $(\mathcal{I}, \Omega)$, it follows that these quantities satisfy the barred versions of the equations $(2.6-8)$, which we will denote by $(2 . \overline{6}-\overline{8})$. Moreover, the eight 1 -form components of $\bar{\lambda}, \bar{\phi}$, and $\bar{\omega}$ are independent on $P$ since $\iota^{*}(\Omega) \neq 0$ and hence they form a coframing on $P$.

For any element $v=v^{0}+v^{2} \in \mathcal{V}_{0} \oplus \mathcal{V}_{2}$, let $X_{v}$ denote the unique vector field on $P$ which satisfies the equations $(\bar{\lambda}+\bar{\phi})\left(X_{v}\right)=v$ and $\bar{\omega}\left(X_{v}\right)=0$. The equations $(2 . \overline{6}-\overline{8})$ then yield the formulas

$$
\begin{aligned}
& \mathfrak{L}_{X_{v}} \bar{\omega}=-v^{0} \bar{\omega}-\left\langle v^{2}, \bar{\omega}\right\rangle_{1} \\
& \mathfrak{L}_{X_{v}} \bar{\lambda}=0 \\
& \mathfrak{L}_{X_{v}} \bar{\phi}=-\left\langle v^{2}, \bar{\phi}\right\rangle_{1} \\
& \mathfrak{L}_{X_{v}} \bar{a}^{i}=2 v^{0} \bar{a}^{i}-\left\langle v^{2}, \bar{a}^{i}\right\rangle_{1} \\
& \mathfrak{L}_{X_{v}} \bar{b}^{i}=3 v^{0} \bar{b}^{j}-\left\langle v^{2}, \bar{b}^{j}\right\rangle_{1}
\end{aligned}
$$

where $\mathfrak{L}_{X_{v}}$ denotes the Lie derivative with respect to $X_{v}$. From these formulas, it follows easily that the vector fields $\left\{X_{v} \mid v \in \mathcal{V}_{0} \oplus \mathcal{V}_{2}\right\}$ generate a locally free right action of the group $G L^{+}(2, \mathbb{R})$ on $P$ in such a way that $\iota=\bar{a}+\bar{b}$ is equivariant with respect to the natural right $G L^{+}(2, \mathbb{R})$ action on $\mathcal{V} \oplus \mathcal{W}$ generated by the right action on each summand given by $R_{g}(u)=g^{-1} \cdot u$ for $u \in \mathcal{V}_{p}$. In particular, note that, for each $z \in P$, the intersection $P \cap\left(z \cdot G L^{+}(2, \mathbb{R})\right)$ is of dimension 4 and is an open subset of the orbit $z \cdot G L^{+}(2, \mathbb{R})$. 
Now let $z_{0} \in P$ be fixed. It follows from the above discussion that there is a neighborhood $U$ of $z_{0}$ in $P$ which can be written in the form $U=M \cdot V$ where $M \subset P$ is a connected submanifold of dimension 4 which passes through $z_{0}$ and which is transverse to the local $G L^{+}(2, \mathbb{R})$ orbits and $V$ is a connected open neighborhood of the identity in $G L^{+}(2, \mathbb{R})$. Let $\bar{\pi}: U \rightarrow M$ be the projection onto the first factor. Let $\pi: \mathcal{F} \rightarrow M$ denote, as usual, the $\operatorname{GL}\left(\mathcal{V}_{3}\right)$ coframe bundle of $M$. There is a natural mapping $\tau: U \rightarrow \mathcal{F}$ which is defined as follows: For $u \in U$, we let $\tau(u)$ denote the linear isomorphism $\tau(u): T_{\bar{\pi}(u)} \stackrel{\sim}{\rightarrow} \mathcal{V}_{3}$ which satisfies $\bar{\omega}(v)=\tau(u)\left(\bar{\pi}_{*}(v)\right)$ for all $v \in T_{u} U$.

It is now easy to check that $\tau$ is a $G L^{+}(2, \mathbb{R})$-equivariant embedding and that there exists a unique $G_{3}$-structure $F$ on $M$ which contains $\tau(U)$ (as an open subset). Moreover, if $\omega$ represents the tautological form on $F$, then $\tau^{*}(\omega)=\bar{\omega}$. It follows easily that if $\phi$ and $\lambda$ represent the intrinsic connection and torsion on $F$, then they satisfy $\tau^{*}(\phi)=\bar{\phi}$ and $\tau^{*}(\lambda)=\bar{\lambda}$. We then conclude from the equations $(2 . \overline{6}-\overline{8})$, that $F$ is a torsion-free $G_{3}$-structure. Moreover, we must have $\tau^{*}\left(a^{i}\right)=\bar{a}^{i}$ and $\tau^{*}\left(b^{j}\right)=\bar{b}^{j}$. It then follows that $\left.\iota\right|_{U}=\bar{a}+\bar{b}=K \circ \tau$, where $K=a+b$ is the curvature mapping of $F$. Moreover, since $\bar{a}=a \circ \tau$, we see that $a: F \rightarrow \mathcal{V}$ is an immersion. Thus, $F$ is non-degenerate.

We have shown that $P$ is locally of the form $K(F)$ for a non-degenerate $G_{3}$-structure $F$. Local uniqueness of $F$ up to diffeomorphism can safely be left to the reader.

We now turn to the problem of proving the existence of non-degenerate torsion-free $G_{3}$-structures. By Proposition 3.1, it suffices to prove the existence of integral manifolds of $(\mathcal{I}, \Omega)$.

As a first step in this analysis, let us define, for each $i=1,3,5$, or 7 , the $\mathcal{V}_{i^{-}}$ valued 1-form $\beta^{i}$ on $\mathcal{O}$ by "emboldening" the definition of $\beta^{i}$ in (2.9). For example, $\boldsymbol{\beta}^{i}=d \mathbf{b}^{7}-3 \boldsymbol{\lambda} \wedge \mathbf{b}^{7}+\left\langle\boldsymbol{\phi}, \mathbf{b}^{7}\right\rangle_{1}$. We will also use the notation $\boldsymbol{\beta}=\boldsymbol{\beta}^{1}+\boldsymbol{\beta}^{3}+\boldsymbol{\beta}^{5}+\boldsymbol{\beta}^{7}$.

If we differentiate $\left(1^{*}\right)$, then, after a considerable amount of linear algebra, we can express the result in the form

$$
\mathbf{A}(\mathbf{a})(\boldsymbol{\Lambda}+\boldsymbol{\Phi})+\mathbf{B}(\mathbf{b})(\boldsymbol{\Theta})=-\mathbf{B}(\boldsymbol{\beta})(\boldsymbol{\omega}) .
$$

Since $\mathbf{J}(\boldsymbol{\Lambda}+\mathbf{\Phi}+\boldsymbol{\Theta})=\mathbf{A}(\mathbf{a})(\boldsymbol{\Lambda}+\boldsymbol{\Phi})+\mathbf{B}(\mathbf{b})(\boldsymbol{\Theta})$ and $\mathbf{J}$ is invertible on $\mathcal{O}$, it follows that the differential system $\mathcal{I}$ can equally well be generated by the eight components of the $\left(\mathcal{V}_{2} \oplus \mathcal{V}_{4}\right)$-valued 2-form $\mathbf{B}(\boldsymbol{\beta})(\boldsymbol{\omega})$.

Proposition 3.2: The differential system with independence condition $(\mathcal{I}, \Omega)$ is in linear form and involutive on $\mathcal{O}$ with Cartan characters $s_{0}=0, s_{1}=s_{2}=8, s_{3}=4$ and $s_{i}=0$ for $i>3$.

Proof: According to the discussion just above, $\mathcal{I}$ is generated by the components of the 2 -forms $\Upsilon^{2}$ and $\Upsilon^{4}$ where

$$
\begin{array}{ll}
\boldsymbol{\Upsilon}^{2}=10\left\langle\boldsymbol{\beta}^{1}, \boldsymbol{\omega}\right\rangle_{1}+\left\langle\boldsymbol{\beta}^{3}, \boldsymbol{\omega}\right\rangle_{2} & +14\left\langle\boldsymbol{\beta}^{5}, \boldsymbol{\omega}\right\rangle_{3} \\
\boldsymbol{\Upsilon}^{4}=9\left\langle\boldsymbol{\beta}^{1}, \boldsymbol{\omega}\right\rangle_{0} & -5\left\langle\boldsymbol{\beta}^{5}, \boldsymbol{\omega}\right\rangle_{2}+\left\langle\boldsymbol{\beta}^{7}, \boldsymbol{\omega}\right\rangle_{3} .
\end{array}
$$

This clearly shows that $\mathcal{I}$ is in linear form. 
Now, according the calculation done at the end of $\S 2$ which resulted in (2.11), the space of integral elements of $(\mathcal{I}, \Omega)$ at each point of $\mathcal{O}$ is an affine space of dimension $S=36$.

It remains to calculate the Cartan characters. Relative to the sequence

$$
\sigma=\left(\boldsymbol{\omega}_{-3}, \boldsymbol{\omega}_{3}, \boldsymbol{\omega}_{-1}, \boldsymbol{\omega}_{1}, \boldsymbol{\lambda}_{0}, \boldsymbol{\phi}_{-2}, \boldsymbol{\phi}_{0}, \boldsymbol{\phi}_{2}\right)
$$

(note the ordering), the reduced character sequence is easily shown to be $s_{0}^{\prime}=0, s_{1}^{\prime}=s_{2}^{\prime}=$ $8, s_{3}^{\prime}=4$ and $s_{i}^{\prime}=0$ for $i>3$. Since $S=36=s_{1}^{\prime}+2 s_{2}^{\prime}+3 s_{3}^{\prime}+4 s_{4}^{\prime}+5 s_{5}^{\prime}+6 s_{6}^{\prime}+7 s_{7}^{\prime}+8 s_{8}^{\prime}$, it follows that Cartan's Test is verified, so the sequence $\sigma$ is regular

Thus, $(\mathcal{I}, \Omega)$ is involutive. Moreover, $s_{i}=s_{i}^{\prime}$ for $0 \leq i \leq 8$.

Theorem 3.3: There exist torsion-free, non-degenerate $G_{3}$-structures whose holonomy is equal to $G_{3}$. In fact, modulo diffeomorphism, the general such structure depends on four functions of three variables.

Proof: It is clear that, for an open set $U$ of values $a=a^{2}+a^{4} \in \mathcal{V}_{2} \oplus \mathcal{V}_{4}$, the associated curvature tensor in $\mathcal{K}\left(\mathfrak{g}_{3}\right)$ does not lie in $\mathcal{K}(\mathfrak{g})$ for any proper subalgebra $\mathfrak{g} \subset \mathfrak{g}_{3}$. Thus, if $F$ is a torsion-free, non-degenerate $G_{3}$-structure on a 4-manifold $M$ whose curvature function $a$ takes values in $U$, then the holonomy of $F$ will be equal to $G_{3}$.

By the Cartan-Kähler theorem, since the differential system $(\mathcal{I}, \Omega)$ is involutive and real-analytic on $\mathcal{O}$, there exist integral manifolds of $(\mathcal{I}, \Omega)$ passing through every point of $\mathcal{O}$. It follows easily that there exists a torsion-free, non-degenerate $G_{3}$-structure on a 4-manifold $M$ whose curvature function $a$ takes values in $U$. The statement about the generality of such structures up to diffeomorphism follows immediately from Proposition 3.2 in which it was shown that the last non-zero character of $(\mathcal{I}, \Omega)$ is $s_{3}=4$.

Note that, in view of Proposition 2.2, one effect of Theorem 3.3 is to prove that there do indeed exist torsion-free affine connections whose holonomy is $G_{3}$.

Before leaving the differential system $\mathcal{I}$, let us note that it has four-dimensional Cauchy characteristics, namely the orbits of the action of $G L^{+}(2, \mathbb{R})$ on $\mathcal{O}$. Unfortunately, this action is not free, so passing to the orbit space will not generally yield a smooth manifold, only an orbifold. However, it is not difficult to show that the generic point of $\mathcal{O}$ has trivial $G L^{+}(2, \mathbb{R})$-stabilizer.

Let $\mathcal{O}^{*} \subset \mathcal{O}$ denote the open set on which $G L^{+}(2, \mathbb{R})$ acts freely. The quotient $X^{*}=\mathcal{O}^{*} / G L^{+}(2, \mathbb{R})$ is then a smooth manifold. If we let $\pi: \mathcal{O}^{*} \rightarrow X^{*}$ denote the canonical projection, then the fibers of $\boldsymbol{\pi}$ are the Cauchy characteristics of the system $\mathcal{I}$. Thus, there is an exterior differential system with independence condition $(\overline{\mathcal{I}}, \bar{\Omega})$ on $X^{*}$ whose local integral manifolds are of dimension 4 and are the $G L^{+}(2, \mathbb{R})$-quotients of the local integral manifolds of $(\mathcal{I}, \Omega)$. This "reduced" system is the natural one for discussing the geometry of the integral manifolds. However, working directly on this space is awkward because there is no natural basis for the generators of $(\overline{\mathcal{I}}, \bar{\Omega})$.

We will close our discussion of the $G_{3}$ case in this section by briefly describing the characteristic variety of the integral manifolds. 
Although the action of $\operatorname{SL}(2, \mathbb{R})$ on $\mathcal{V}_{3}$ does not preserve any symmetric quadratic or cubic forms, it does preserve a quartic form $Q$ given by $Q(v)=\left\langle\langle v, v\rangle_{2},\langle v, v\rangle_{2}\right\rangle_{2}$ for all $v \in \mathcal{V}_{3}$. It is easy to see that $Q(v)=0$ if and only if $v$ has a double or triple linear factor when regarded as a homogeneous cubic polynomial in $x$ and $y$. Thus, $Q$ is essentially the discriminant of $v$.

If a 4-manifold $M$ is endowed with a $G_{3}$-structure $F$, then each tangent space $T_{x} M$ can be identified with $\mathcal{V}_{3}$ up to an action of $G_{3}$, and thus, the polynomial $Q$ is well-defined up to a scalar multiple on $T_{x} M$. In particular, $\Xi_{x} \subset \mathbb{P}\left(T_{x} M\right)$, the zero locus of $Q$, is a well defined algebraic hypersurface of degree 4 . The union of all of these varieties as $x \in M$ varies is a subset $\Xi \subset \mathbb{P}(T M)$. It is not difficult to show that if $F$ is a torsion-free, nondegenerate $G_{3}$-structure, then $\Xi$ is the characteristic variety of the corresponding integral of the Cauchy-reduced exterior differential system $\overline{\mathcal{I}}$.

We now turn to the case of $H_{3}$. The remainder of this section will be devoted to a proof that torsion-free $H_{3}$-structures do, indeed, exist. Moreover, in a certain sense, the moduli space of torsion-free $H_{3}$-structures modulo diffeomorphism will be shown to consist of the disjoint union of a two-dimensional space, two one-dimensional spaces, and four points.

It is natural to attempt an analysis similar to the one which worked for $G_{3}$. However, due to a remarkable identity to be described below, the $H_{3}$ situation is quite different.

Let $F$ be a torsion-free $H_{3}$-structure on a 4-manifold $M$. Using the notation from $\S 2$, there are well-defined functions $a$ and $b$ on $F$ with values in $\mathcal{V}_{2}$ and $\mathcal{V}_{3}$ respectively. Note that, under the homothety action, we have $R_{t}{ }^{*}\left(a^{t}\right)=t^{2} a, R_{t}{ }^{*}\left(b^{t}\right)=t^{3} b$, and $R_{t}{ }^{*}\left(c^{t}\right)=t^{4} c$. Thus, we will assign the functions $a, b$, and $c$ the weights 2,3 , and 4 , respectively. Similarly, we assign $\omega$ and $\phi$ the respective weights -1 and 0 . Of course, by homothety, we could now reduce to the cases where $c=-1$, 0 , or 1 , but this does not significantly simplify the calculations, so we will carry $c$ along as a parameter.

For simplicity of notation, let us set $\mathcal{V}=\mathcal{V}_{2} \oplus \mathcal{V}_{3}$ and let $\mathbf{a}: \mathcal{V} \rightarrow \mathcal{V}_{2}$ and $\mathbf{b}: \mathcal{V} \rightarrow \mathcal{V}_{3}$ denote the projections thought of as vector-valued functions on $\mathcal{V}$. If we set $K=a+b$, then $K: F \rightarrow \mathcal{V}$ is a $\operatorname{SL}(2, \mathbb{R})$-equivariant mapping satisfying $K^{*}(\mathbf{a})=a$ and $K^{*}(\mathbf{b})=b$. Since both $F$ and $\mathcal{V}$ have dimension 7 , one might hope to use this mapping and the structure equations (2.3-4) to embed (or at least immerse) $F$ into $\mathcal{V}$ and then mimic the procedure which worked for $G_{3}$.

However, this fails for the following reason: Let us write (2.3-4) in the form

$$
d a+d b=J(\phi+\omega)
$$

where $J$ is a function on $F$ with values in $\operatorname{hom}(\mathcal{V}, \mathcal{V})$. Now $J=K^{*}\left(\mathbf{J}_{c}\right)$ where $\mathbf{J}_{c}: \mathcal{V} \rightarrow$ $\operatorname{hom}(\mathcal{V}, \mathcal{V})$ is a polynomial mapping which depends upon a real parameter $c$. Relative to the standard basis $\left(x^{2}, x y, y^{2}, x^{3}, x^{2} y, x y^{2}, y^{3}\right)$ of $\mathcal{V}$, the linear transformation $\mathbf{J}_{c}$ has the 
matrix representation

$$
\mathbf{J}_{c}=\left(\begin{array}{ccccccc}
-2 \mathbf{a}_{0} & 2 \mathbf{a}_{-2} & 0 & 6 \mathbf{b}_{1} & -4 \mathbf{b}_{-1} & 6 \mathbf{b}_{-3} & 0 \\
-4 \mathbf{a}_{2} & 0 & 4 \mathbf{a}_{-2} & 18 \mathbf{b}_{3} & -2 \mathbf{b}_{1} & -2 \mathbf{b}_{-1} & 18 \mathbf{b}_{-3} \\
0 & -2 \mathbf{a}_{2} & 2 \mathbf{a}_{0} & 0 & 6 \mathbf{b}_{3} & -4 \mathbf{b}_{1} & 6 \mathbf{b}_{-1} \\
-2 \mathbf{b}_{-1} & 3 \mathbf{b}_{-3} & 0 & c-\mathbf{q}_{1} & -\mathbf{a}_{-2} \mathbf{a}_{0} & \mathbf{a}_{-2}^{2} & 0 \\
-4 \mathbf{b}_{1} & \mathbf{b}_{-1} & 6 \mathbf{b}_{-3} & 3 \mathbf{a}_{0} \mathbf{a}_{2} & c-\mathbf{q}_{2} & 0 & 3 \mathbf{a}_{-2}^{2} \\
-6 \mathbf{b}_{3} & -\mathbf{b}_{1} & 4 \mathbf{b}_{-1} & 3 \mathbf{a}_{2}^{2} & 0 & c-\mathbf{q}_{2} & 3 \mathbf{a}_{-2} \mathbf{a}_{0} \\
0 & -3 \mathbf{b}_{3} & 2 \mathbf{b}_{1} & 0 & \mathbf{a}_{2}^{2} & -\mathbf{a}_{0} \mathbf{a}_{2} & c-\mathbf{q}_{1}
\end{array}\right)
$$

where $\mathbf{q}_{1}=3 \mathbf{a}_{-2} \mathbf{a}_{2}-\frac{3}{2} \mathbf{a}_{0}^{2}$ and $\mathbf{q}_{2}=5 \mathbf{a}_{-2} \mathbf{a}_{2}-\frac{1}{2} \mathbf{a}_{0}^{2}$.

If $J$ were invertible, then we could regard the components of the functions $a$ and $b$ as a natural coordinate system on $F$ and define the forms $\phi$ and $\omega$ by the formula $(\phi+\omega)=J^{-1}(d a+d b)$. However, by direct MAPLE calculation, one reaches the remarkable conclusion that $\operatorname{det}\left(\mathbf{J}_{c}\right) \equiv 0$.

Thus, we must seek other methods for determining whether there exist local 1-forms $\phi$ and $\omega$ with linearly independent components satisfying (2.1-4) on open domains in $\mathbb{R}^{7}$ for any value of the constant $c$. This class of problem was addressed by É. Cartan via his generalization of the third fundamental theorem of Lie, (Cartan [1904]). However, appealing to Cartan's general results at this point is somewhat unsatisfactory. First, his treatment is rather sketchy in the "intransitive" case (he does not even state a theorem explicitly) and this is the case into which our problem falls. Second, in our case, much more information can be got by direct methods.

Since $\operatorname{det}\left(\mathbf{J}_{c}\right) \equiv 0$, it follows that, if we let $\mathbf{L}_{c}$ denote the classical adjoint matrix of $\mathbf{J}_{c}$ (i.e., the matrix of 6-by-6 minors), then $\mathbf{L}_{c} \mathbf{J}_{c}=\mathbf{J}_{c} \mathbf{L}_{c}=0$. Computation of $\mathbf{L}_{c}$ as a matrix with entries which are polynomial in the quantities $\mathbf{a}, \mathbf{b}$, and $c$ shows that $\mathbf{L}_{c}$ does not vanish identically. (The reader should be careful to distinguish $L=K^{*}\left(\mathbf{L}_{c}\right)$ from $\mathbf{L}_{c}$, for it can happen that $L$ vanishes identically.) Let $\Sigma_{c} \subset \mathcal{V}$ denote the subset on which $\mathbf{L}_{c}$ vanishes. For $\mathbf{x} \in \mathcal{V} \backslash \Sigma_{c}$, the linear mapping $\mathbf{J}_{c}(\mathbf{x}): \mathcal{V} \rightarrow \mathcal{V}$ has rank 6 and the mapping $\mathbf{L}_{c}$ has rank 1 .

It is a standard (and easy) result in commutative algebra that a rectangular matrix with polynomial entries which has rank at most 1 for all values of the indeterminates in the polynomials can be factored into the product of a column and a row, each of whose entries are also polynomials. This applies to $\mathbf{L}_{c}$ to show that there must exist polynomial mappings $\mathbf{r}_{c}: \mathcal{V} \rightarrow \mathcal{V}$ and $\boldsymbol{\rho}_{c}: \mathcal{V} \rightarrow \mathcal{V}^{*}$ so that $\mathbf{L}_{c}(v)=\boldsymbol{\rho}_{c}(v) \mathbf{r}_{c}$ for all $v \in \mathcal{V}$. Of course, actually finding $\mathbf{r}_{c}$ and $\boldsymbol{\rho}_{c}$ is an enormous algebra problem which requires factoring the entries of $\mathbf{L}_{c}$.

Just this once, we will comment on the mechanical calculations: The typical entry of $\mathbf{L}_{c}$ has between 150 and 200 terms in the $\mathbf{a}$ and $\mathbf{b}$ variables. For some inexplicable reason having to do with the algorithm used to find factors, MAPLE was unable to factor about half of the entries. Fortunately, as the reader will have realized, it suffices to factor at least one entry in each row and column. This MAPLE was able to do.

The typical entry is the product of two irreducible factors of degree 5 . In the cases where MAPLE was able to factor the entry, the process took about 10 to 15 minutes on 
a Macintosh SE/30. When MAPLE failed to find a factorization, it typically reported failure or returned errors in about 5 minutes. For a SUN 3/60 implementation of the same problem, corresponding processes were about four times as fast. Reassuringly(?), failures and errors were reported for the same entries on both machines.

Once enough entries of $\mathbf{L}_{c}$ had been factored to determine candidates for $\mathbf{r}_{c}$ and $\boldsymbol{\rho}_{c}$, factorizations of the remaining entries were easily checked by multiplication.

The result of this calculation can be described as follows: Let $\mathbf{p}_{c}: \mathcal{V} \rightarrow \mathcal{V}_{2}$, q: $\mathcal{V} \rightarrow \mathcal{V}_{1}$, $\mathbf{r}_{c}^{2}: \mathcal{V} \rightarrow \mathcal{V}_{2}$, and $\mathbf{r}_{c}^{3}: \mathcal{V} \rightarrow \mathcal{V}_{3}$ be the following polynomial functions:

$$
\begin{aligned}
& \mathbf{p}_{c}=\left(2 c-\langle\mathbf{a}, \mathbf{a}\rangle_{2}\right) \mathbf{a}-\langle\mathbf{b}, \mathbf{b}\rangle_{2} \\
& \mathbf{q}=\langle\mathbf{a}, \mathbf{b}\rangle_{2} \\
& \mathbf{r}_{c}^{2}=\frac{7}{6}\langle\mathbf{q}, \mathbf{q}\rangle_{0}+\frac{1}{3}\left\langle\langle\mathbf{a}, \mathbf{b}\rangle_{1}, \mathbf{q}\right\rangle_{1}+\frac{1}{2}\left(2 c-\langle\mathbf{a}, \mathbf{a}\rangle_{2}\right) \mathbf{p}_{c}-\left\langle\mathbf{a}, \mathbf{p}_{c}\right\rangle_{2} \mathbf{a} \\
& \mathbf{r}_{c}^{3}=-\left\langle\mathbf{b}, \mathbf{p}_{c}\right\rangle_{1}-\mathbf{a}\langle\mathbf{a}, \mathbf{q}\rangle_{1} .
\end{aligned}
$$

Then for all $v=v^{2}+v^{3} \in \mathcal{V}$ with $v^{i} \in \mathcal{V}^{i}$, we have

$$
\mathbf{L}_{c}(v)=\left(\left\langle\mathbf{r}_{c}^{2}, v^{2}\right\rangle_{2}-\left\langle\mathbf{r}_{c}^{3}, v^{3}\right\rangle_{3}\right)\left(\mathbf{r}_{c}^{2}+\mathbf{r}_{c}^{3}\right)
$$

In particular, note that

$$
\Sigma_{c}=\left\{\mathbf{x} \in \mathcal{V} \mid \mathbf{r}_{c}^{2}(\mathbf{x})=\mathbf{r}_{c}^{3}(\mathbf{x})=0\right\}
$$

Now a second remarkable identity occurs: If we set

$$
\boldsymbol{\rho}_{c}=\left\langle\mathbf{r}_{c}^{2}, d \mathbf{a}\right\rangle_{2}-\left\langle\mathbf{r}_{c}^{3}, d \mathbf{b}\right\rangle_{3},
$$

then the 1-form $\boldsymbol{\rho}_{c}$ is closed. In particular, by the polynomial Poincaré lemma, there must exist a polynomial $\mathbf{R}_{c}$ on $\mathcal{V}$ so that $d \mathbf{R}_{c}=\boldsymbol{\rho}_{c}$. If we normalize this polynomial by requiring that it vanish at $0 \in \mathcal{V}$, then

$$
\mathbf{R}_{c}=\frac{1}{4}\left\langle\mathbf{p}_{c}, \mathbf{p}_{c}\right\rangle_{2}+\frac{1}{2}\left\langle\mathbf{a},\langle\mathbf{q}, \mathbf{q}\rangle_{0}\right\rangle_{2}
$$

This function $\mathbf{R}_{c}$ has the following significance: By construction, its differential $\boldsymbol{\rho}_{c}$ at each point $\mathbf{x} \in \mathcal{V}$ is a linear form on $\mathcal{V}$ whose kernel contains the image of $\mathbf{J}_{c}(\mathbf{x})$. Since $K=a+b=J(\phi+\omega)=K^{*}\left(\mathbf{J}_{c}\right)(\phi+\omega)$, it follows that for each $f \in F$, the image of the differential mapping $d K_{f}: T_{f} F \rightarrow T_{K(f)} \mathcal{V}$ lies in the kernel of $\boldsymbol{\rho}_{c}$ at $K(f)$. In other words, $K^{*}\left(\boldsymbol{\rho}_{c}\right)=0$. Thus, $K$ maps $F$ into a level set of $\mathbf{R}_{c}$.

(Upon seeing this, the reader may well wonder why it is so remarkable that $\boldsymbol{\rho}_{c}$ is closed. After all, if, for every point $\mathrm{x} \in \mathcal{V}$, there were an $H_{3}$-structure $F$ whose curvature mapping $K$ covered $\mathbf{x}$ and whose differential had rank 6 , then $\mathcal{V}$ would be foliated by codimension 1 integral manifolds of $\boldsymbol{\rho}_{c}$. This would, of course, imply that $\boldsymbol{\rho}_{c} \wedge d \boldsymbol{\rho}_{c} \equiv 0$, i.e., that $\boldsymbol{\rho}_{c}$ was integrable. However, this is still a long way from knowing that $\boldsymbol{\rho}_{c}$ is closed. An integrable polynomial 1 -form $\theta$ on a vector space $V$ cannot generally be written (even locally) in the form $\theta=f d g$ where $g$ is a polynomial function on $V$.) 
Let $r^{2}=K^{*}\left(\mathbf{r}_{c}^{2}\right)$ and $r^{3}=K^{*}\left(\mathbf{r}_{c}^{3}\right)$. Since the 7 components of $\phi$ and $\omega$ form a coframing of $F$, it follows that there exists a unique vector field $Z$ on $F$ for which we have the identities $\phi(Z)=r^{2}$ and $\omega(Z)=r^{3}$. By (9), (12) and the fact that $L J=0$, it follows that $Z(u)$ lies in the kernel of the mapping $d K_{u}: T_{u} F \rightarrow T_{K(u)} \mathcal{V}$ for all $u \in F$. In particular, $a$ and $b$ are constant on the integral curves of $Z$.

Another calculation now reveals a third remarkable identity:

$$
\mathfrak{L}_{Z} \phi=\mathfrak{L}_{Z} \omega=0
$$

(Again, the knowledgeable reader may wonder why this is remarkable. After all, a general theorem of Cartan [1904] implies that, locally on $F$, away from the singularities of $Z$, there exists a multiplier $\mu$ so that the equations (16) are satisfied with $Z$ replaced by $\mu Z$. However, the general theory does not provide a method for finding $\mu$ other than integrating a (linear) system of ODE. Luckily, in this case $\mu=1$ happens to work.)

It follows from (16) that the $\mathcal{V}$-valued coframing $\phi+\omega$ on $F$ is preserved by the flow of $Z$. Of course, this implies that either $Z$ vanishes identically or else $Z$ never vanishes. (Remember that we are assuming that $M$ (and hence $F$ ) is connected.) We shall say that a torsion-free $H_{3}$-structure is regular if $Z$ never vanishes and singular if $Z$ is identically zero.

For any regular torsion-free $H_{3}$-structure $F$ on $M$, the rank of the linear map $J$ is 6 at every point of $F$. In particular, for a regular $F$ structure, the curvature mapping $K: F \rightarrow \mathcal{V}$ is a submersion into a level set of $\mathbf{R}_{c}$ in $\mathcal{V} \backslash \Sigma_{c}$. Moreover, the local flow of the vector field $Z$ fixes both $\phi$ and $\omega$. Thus, we say that $Z$ is an infinitesimal symmetry of the coframing $(\phi, \omega)$. Up to constant scalar multiples, $Z$ is the only infinitesimal symmetry of $(\phi, \omega)$. This is because any infinitesimal symmetry $Y$ would be tangent to the (one-dimensional) fibers of $K: F \rightarrow \mathcal{V}$ and hence be of the form $\mu Z$ for some function $\mu$. However, it is easy to see that $\mu Z$ is an infinitesimal symmetry only if $\mu$ is constant.

Of course, the infinitesimal symmetries of $(\phi, \omega)$ on $F$ are in one-to-one correspondence with the vector fields on $M$ whose flows preserve the $H_{3}$-structure $F$, so it follows that any regular torsion-free $H_{3}$-structure on $M$ has a one-dimensional local automorphism group.

We are now ready for the following fundamental theorem:

TheOrem 3.4: Let $c$ be any constant and let $\mathcal{C} \subset \mathcal{V} \backslash \Sigma_{c}$ be any level set of $\mathbf{R}_{c}$ in $\mathcal{V} \backslash \Sigma_{c}$. Then $\mathcal{C}$ can be covered by open subsets $U$ which have the following property: There exists a principal $\mathbb{R}$-bundle $K: F_{U} \rightarrow U$ over $U$ and 1 -forms $\phi$ and $\omega$ on $F_{U}$, with values in $\mathcal{V}_{2}$ and $\mathcal{V}_{3}$ respectively, satisfying

(i) $\phi+\omega$ is a $\mathcal{V}$-valued coframing on $F_{U}$

(ii) The structure equations (2.1-4) are satisfied with $a=K^{*}(\mathbf{a})$ and $b=K^{*}(\mathbf{b})$.

Moreover, the triple $\left(F_{U}, \phi, \omega\right)$ is unique in the sense that any other $\mathbb{R}$-bundle $F^{\prime}$ over $U$ equipped with a $\mathcal{V}$-valued coframing $\phi^{\prime}+\omega^{\prime}$ satisfying $(i)$ and $(i i)$ is bundle equivalent to $F_{U}$ via an equivalence which identifies the coframings.

Proof: Let $\bar{a}, \bar{b}, \bar{r}^{2}, \bar{r}^{3}$, and $\bar{J}$ denote the restrictions of the functions $\mathbf{a}, \mathbf{b}, \mathbf{r}^{2}, \mathbf{r}^{3}$, and $\mathbf{J}_{c}$, respectively, to $\mathcal{C}$. 
We claim that there exist 1 -forms $\bar{\phi}$ and $\bar{\omega}$ on $\mathcal{C}$ with values in $\mathcal{V}_{2}$ and $\mathcal{V}_{3}$ respectively which satisfy the equation

$$
d \bar{a}+d \bar{b}=\bar{J}(\bar{\phi}+\bar{\omega})
$$

To see this, first note that since $\mathcal{C}$ lies in $\mathcal{V} \backslash \Sigma_{c}$, it follows that $\bar{J}$ has rank 6 at every point of $\mathcal{C}$. Moreover, by construction, at each point of $\mathcal{C}$, the 1 -form $d \bar{a}+d \bar{b}$ takes values in the 6 -dimensional subspace of $\mathcal{V}$ which is the image of $\bar{J}$. It follows that, as an inhomogeneous system of linear equations for $(\bar{\phi}, \bar{\omega})$, the system (17) has a one-parameter family of solutions at each point of $\mathcal{C}$. Because $\bar{J}$ has constant rank 6 , it follows that (17) can be solved smoothly for $(\bar{\phi}, \bar{\omega})$.

The kernel of $\bar{J}$ is spanned at each point by the vector $\bar{r}^{2}+\bar{r}^{3}$, so once one solution $(\bar{\phi}, \bar{\omega})$ to $(17)$ has been found, any other can be written in the form $\left(\bar{\phi}+\bar{r}^{2} \alpha, \bar{\omega}+\bar{r}^{3} \alpha\right)$ for some unique 1 -form $\alpha$ on $\mathcal{C}$.

It is worth remarking that the equations (17) can be expressed in the expanded form:

$$
\begin{aligned}
& d \bar{a}=-\langle\bar{\phi}, \bar{a}\rangle_{1}+\langle\bar{b}, \bar{\omega}\rangle_{2} \\
& d \bar{b}=-\langle\bar{\phi}, \bar{b}\rangle_{1}+\left(c-\langle\bar{a}, \bar{a}\rangle_{2}\right) \bar{\omega}++\frac{1}{12}\left\langle\langle\bar{a}, \bar{a}\rangle_{0}, \bar{\omega}\right\rangle_{2} .
\end{aligned}
$$

Using the explicit formulas for $\bar{r}^{2}$ and $\bar{r}^{3}$ in terms of $\bar{a}$ and $\bar{b}$ derived from (11), it can be calculated that

$$
\begin{aligned}
& d \bar{r}^{2}=-\left\langle\bar{\phi}, \bar{r}^{2}\right\rangle_{1}-2 \bar{a}\left\langle\bar{r}^{3}, \bar{\omega}\right\rangle_{3}+\frac{1}{6}\left\langle\bar{a},\left\langle\bar{r}^{3}, \bar{\omega}\right\rangle_{1}\right\rangle_{2} \\
& d \bar{r}^{3}=-\left\langle\bar{\phi}, \bar{r}^{3}\right\rangle_{1}+\left\langle\bar{r}^{2}, \bar{\omega}\right\rangle_{1} .
\end{aligned}
$$

Now define the 2-forms

$$
\begin{aligned}
& \bar{\Theta}=d \bar{\omega}+\langle\bar{\phi}, \bar{\omega}\rangle_{1} \\
& \bar{\Phi}=d \bar{\phi}+\frac{1}{2}\langle\bar{\phi}, \bar{\phi}\rangle_{1}-\bar{a}\langle\bar{\omega}, \bar{\omega}\rangle_{3}+\frac{1}{12}\left\langle\bar{a},\langle\bar{\omega}, \bar{\omega}\rangle_{1}\right\rangle_{2} .
\end{aligned}
$$

After some calculation, the exterior derivative of (17) can be written in the form $0=$ $\bar{J}(\bar{\Phi}+\bar{\Theta})$. Of course, since the kernel of $\bar{J}$ is spanned by $\bar{r}^{2}+\bar{r}^{3}$, this implies that there exists a 2 -form $\bar{\Psi}$ so that $\bar{\Phi}=\bar{r}^{2} \bar{\Psi}$ and $\bar{\Theta}=\bar{r}^{3} \bar{\Psi}$. Substituting these relations into (19), differentiating, and using the relations (18), we compute that $\bar{r}^{2} d \bar{\Psi}=\bar{r}^{3} d \bar{\Psi}=0$. This implies that $d \bar{\Psi}=0$.

Now let $U \subset \mathcal{C}$ be any open set on which there exists a 1-form $\alpha$ satisfying $d \alpha=$ $-\bar{\Psi}$. Clearly, $\mathcal{C}$ can be covered by such open sets. Replacing the pair $(\bar{\phi}, \bar{\omega})$ by the pair $\left(\bar{\phi}+\bar{r}^{2} \alpha, \bar{\omega}+\bar{r}^{3} \alpha\right)$, we may compute that, for this new pair, we have $\bar{\Phi}=\bar{\Theta}=0$, so we suppose this from now on. In other words, $(\bar{\phi}, \bar{\omega})$ satisfy the structure equations $(2.1-4)$.

Now, let $F_{U}=\mathbb{R} \times U$, let $t$ denote a coordinate on the first factor, and let $K: F_{U} \rightarrow U$ denote projection onto the second factor. Let $a, b, r^{2}, r^{3}$, and $J$ denote the functions $\bar{a}, \bar{b}$, $\bar{r}^{2}, \bar{r}^{3}$, and $\bar{J}$ regarded as functions on $F_{U}$ and define

$$
(\phi, \omega)=\left(\bar{\phi}+\bar{r}^{2} d t, \bar{\omega}+\bar{r}^{3} d t\right)
$$


It is now just a matter of calculation to see that $\left(F_{U}, \phi, \omega\right)$ satisfies all of the conditions of the Theorem. (This heavily uses all sorts of pairing identities as well as the equations $(17-19)$. It is not obvious.).

Finally, the uniqueness follows from the standard facts about mappings preserving coframings, see Gardner [1989].

A corollary of Theorem 3.4 is the existence of regular torsion-free $H_{3}$-structures:

Corollary 3.5: For any constant $c_{0}$ and any point $v \in \mathcal{V} \backslash \Sigma_{c_{0}}$, there exists a regular torsion-free $H_{3}$-structure $F$ on a neighborhood of $0 \in \mathbb{R}^{4}$ with $c=c_{0}$ and a frame $u \in F_{0}$ so that $K(u)=v$. Moreover, $F$ is locally unique up to diffeomorphism.

ProOF: Let $V$ be an open neighborhood of $v$ in the level set of $\mathbf{R}_{c_{0}}$ which contains $v$ and to which Theorem 3.4 applies, and let $\left(F_{V}, \phi, \omega\right)$ be the corresponding triple. Let $U \subset F_{V}$ be an open neighborhood of a point $u \in F_{V}$ which satisfies $K(u)=v$.

By the structure equations, the rank 4 Pfaffian system $I$ generated by the components of $\omega$ is completely integrable on $F_{V}$. It follows that, by shrinking $U$ if necessary, we may suppose that there exists a submersion $\pi: U \rightarrow \mathbb{R}^{4}$ satisfying $\pi(u)=0$ and whose fibers are connected and constitute the leaves of $I$ restricted to $U$.

We claim that there exists a unique immersion $\tau: U \rightarrow \mathcal{F}$, where $\mathcal{F}$ is the $\operatorname{GL}\left(\mathcal{V}_{3}\right)$ coframe bundle of $\mathbb{R}^{4}$ which pulls back the tautological $\mathcal{V}_{3}$-valued 1 -form on $\mathcal{F}$ to become $\omega$ on $U$. Moreover, by shrinking $U$, we may even suppose that $\tau$ is an embedding and that the image is an open subset of an $H_{3}$-structure $F$ on a neighborhood of $0 \in \mathbb{R}^{4}$. Finally, the mapping $\tau$ pulls back the intrinsic connection form on $F$ to become $\phi$ and the constant $c$ to be $c_{0}$. The argument for these claims is exactly analogous to the one in the last paragraph of the proof of Proposition 3.1, so we will not repeat it.

Since the forms $\phi$ and $\omega$ satisfy $(2.1-4)$, it follows that $F$ is torsion-free. Using $\tau$ to identify $U$ with an open set in $F$, it follows that $K(u)=v$ as desired. By construction, $K(F) \subset V \subset \mathcal{V} \backslash \Sigma_{c_{0}}$, so $F$ is regular.

Finally, in order to prove local uniqueness, we appeal to Theorem 3.4 again. If $F^{\prime}$ were another torsion-free $H_{3}$-structure on a neighborhood of $0 \in \mathbb{R}^{4}$ which contained a point $u^{\prime} \in F_{0}^{\prime}$ with $K^{\prime}\left(u^{\prime}\right)=v$ and had $c=c_{0}$, then locally both $K: F \rightarrow \mathcal{V}$ and $K^{\prime}: F^{\prime} \rightarrow \mathcal{V}$ would be submersions into the level set of $\mathbf{R}_{c_{0}}$ which contained $v$ and hence would, by the uniqueness aspect of Theorem 3.4, be locally coframe equivalent. This clearly implies that there exists a local diffeomorphism on a neighborhood of $0 \in \mathbb{R}^{4}$ which carries $F^{\prime}$ to $F$ (see Gardner [1989]).

Of course, Corollary 3.5 is not the ideal statement. One would like to be able to prove that each connected component $\mathcal{C}$ of a level set of $\mathbf{R}_{c}$ in $\mathcal{V} \subset \Sigma_{c}$ is of the form $K\left(F_{\mathcal{C}}\right)$ for some torsion-free $H_{3}$-structure $F_{\mathcal{C}}$ on a connected 4-manifold $M_{\mathcal{C}}$ and that this pair $\left(M_{\mathcal{C}}, F_{\mathcal{C}}\right)$ is unique up to diffeomorphism. If we could do this, then we could begin to make rigorous sense of the claim that, up to diffeomorphism, the regular, torsion-free $H_{3}$-structures form a moduli space of dimension 2 with "coordinates" given by the pair $\left(c, K^{*}\left(\mathbf{R}_{c}\right)\right)$.

Note that a homothety class of torsion-free $H_{3}$-structures in these "coordinates" would be described by a curve of the form $\left(c^{t}, k^{t}\right)=\left(t^{4} c, t^{12} k\right)$, so the moduli space of regular 
homothety classes (and hence, regular torsion-free connections with holonomy conjugate to $\mathrm{H}_{3}$ ) would be one-dimensional.

Unfortunately, because we cannot, as yet, determine the topology of the components $\mathcal{C}$, the step in the proof of Theorem 3.4 where we must assume that the closed 2 -form $\bar{\Psi}$ is exact remains a stumbling block. The best we can do along these lines is state that, if two torsion-free $H_{3}$-structures, $F_{1}$ and $F_{2}$ are constructed via Corollary 3.5 using the same constant $c_{0}$ and points $v_{1}$ and $v_{2}$ which lie on the same connected component $\mathcal{C}$ of a level set of $\mathbf{R}_{c_{0}}$ in $\mathcal{V} \subset \Sigma_{c_{0}}$, then they must be "analytic continuations" of each other in an appropriate sense.

We now turn our attention to the singular case, i.e., where $Z$ vanishes identically. This is equivalent to the condition that $K: F \rightarrow \mathcal{V}$ has its image in $\Sigma_{c}$. This study will therefore require a description of the singular locus $\Sigma_{c}$. Fortunately, because this locus is invariant under the action of $\mathrm{SL}(2, \mathbb{R})$ (and because we can use MAPLE), this description is available.

For $c=0$, it can be shown that $\Sigma_{0}$ is the union of two irreducible four-dimensional components, $\Sigma_{0}^{ \pm}$, which can be described as follows.

$$
\Sigma_{0}^{ \pm}=\left\{\left( \pm \mathbf{v}^{2}+\mathbf{u}^{2}, \mp \mathbf{v}^{2} \mathbf{u}+\frac{1}{3} \mathbf{u}^{3}\right) \in \mathcal{V} \mid \mathbf{u}, \mathbf{v} \in \mathcal{V}_{1}\right\}
$$

These two components are smooth away from their two-dimensional intersection, $\Sigma_{0}^{0}$, given by

$$
\Sigma_{0}^{0}=\left\{\left(\mathbf{u}^{2}, \frac{1}{3} \mathbf{u}^{3}\right) \in \mathcal{V} \mid \mathbf{u} \in \mathcal{V}_{1}\right\} .
$$

Note that $\Sigma_{0}^{0}$ itself is smooth except at the point $(0,0) \in \mathcal{V}$.

Moreover, the matrix $\mathbf{J}_{0}$ has rank 4 on $\Sigma_{0}^{ \pm} \backslash \Sigma_{0}^{0}$, rank 2 on $\Sigma_{0}^{0} \backslash\{(0,0)\}$, and (of course) rank 0 at $(0,0)$.

Now, let us assume that $c \neq 0$. By (11), it is clear that the equations $\mathbf{r}^{2}=\mathbf{r}^{3}=0$ are satisfied on the locus where $\mathbf{p}_{c}$ and $\mathbf{q}$ vanish. Thus, we can define a subset of $\Sigma_{c}$ by

$$
\Sigma_{c}^{1}=\left\{(\mathbf{a}, \mathbf{b}) \in \mathcal{V} \mid\left(2 c-\langle\mathbf{a}, \mathbf{a}\rangle_{2}\right) \mathbf{a}-\langle\mathbf{b}, \mathbf{b}\rangle_{2}=\langle\mathbf{a}, \mathbf{b}\rangle_{2}=0\right\}
$$

For $c \neq 0$, it can be shown that $\Sigma_{c}^{1}$ is irreducible and smooth of dimension 4 . Moreover, the matrix $\mathbf{J}_{c}$ has rank 4 everywhere on $\Sigma_{c}^{1}$.

It turns out that there is one other component $\Sigma_{c}^{2}$ of $\Sigma_{c}$ and it can be parametrized as follows:

$$
\Sigma_{c}^{2}=\left\{\left(\mathbf{v}+\mathbf{u}^{2}, \frac{1}{3} \mathbf{u}^{3}-\mathbf{v u}\right) \in \mathcal{V} \mid \mathbf{u} \in \mathcal{V}_{1}, \mathbf{v} \in \mathcal{V}_{2}, \text { where }\langle\mathbf{v}, \mathbf{v}\rangle_{2}=\frac{2}{3} c\right\}
$$

It can be shown that $\Sigma_{c}^{2}$ is algebraically irreducible and smooth of dimension 4 . (Topologically, $\Sigma_{c}^{2}$ is the product of $\mathbb{R}^{2}$ with an hyperboloid of either two or one sheets depending on whether $c$ is positive or negative.) Moreover, the matrix $\mathbf{J}_{c}$ has rank 4 everywhere on $\Sigma_{c}^{2}$.

Note that, as $c \rightarrow 0$, both $\Sigma_{c}^{1}$ and $\Sigma_{c}^{2}$ reduce to the union of the two components $\Sigma_{0}^{ \pm}$ of $\Sigma_{0}$. 
By an analysis similar to the one carried out in the course of proving Theorem 3.4 and Corollary 3.5, we can arrive at the following theorem which classifies all of the singular torsion-free $H_{3}$-structures. The results of this theorem are more global than those in the regular case since we have a good description of the topology of the singular locus $\Sigma_{c}$. We omit the (straightforward) proof.

THEOREM 3.6: The following singular torsion-free $H_{3}$-structures exist and have the stated properties. Except as noted otherwise, these belong to distinct homothety classes. Moreover, every singular torsion-free $H_{3}$-structure is locally diffeomorphic to exactly one on this list.

(i) For each $c_{0} \neq 0$, an $H_{3}$-structure $F_{c_{0}}^{1}$ on a connected 4-manifold $M_{c_{0}}^{1}$ with the property that $c=c_{0}$ and whose curvature mapping $K: F_{c_{0}}^{1} \rightarrow \mathcal{V}$ is a submersion onto $\Sigma_{c_{0}}^{1}$. The symmetry group of $F_{c_{0}}^{1}$ has dimension 3. The structures $F_{c_{0}}^{1}$ and $F_{c_{1}}^{1}$ belong to the same homothety class if and only if $c_{1}$ and $c_{0}$ have the same sign.

(ii) For each $c_{0} \neq 0$, an $H_{3}$-structure $F_{c_{0}}^{2}$ on a 4 -manifold $M_{c_{0}}^{2}$ with the property that $c=c_{0}$ and whose curvature mapping $K: F_{c_{0}}^{2} \rightarrow \mathcal{V}$ is a submersion onto $\Sigma_{c_{0}}^{2}$. The symmetry group of $F_{c_{0}}^{2}$ has dimension 3. If $c_{0}<0$, then $M_{c_{0}}^{2}$ is connected and all of these $F_{c_{0}}^{2}$ belong to the same homothety class. If $c_{0}>0$, then $M_{c_{0}}^{2}$ has two connected components and each of these components determines a unique homothety class.

(iii) For $\epsilon= \pm$, an $H_{3}$-structure $F^{\epsilon}$ on a 4-manifold $M^{\epsilon}$ with the property that $c=$ 0 and whose curvature mapping $K: F^{\epsilon} \rightarrow \mathcal{V}$ is a submersion onto $\Sigma_{0}^{\epsilon} \backslash \Sigma_{0}^{0}$. The symmetry group of $F^{\epsilon}$ has dimension 3; however, its conformal symmetry group has dimension 4 and has an open orbit on $M^{\epsilon}$. Moreover, each of $F^{+}$and $F^{-}$is the unique representative in its homothety class.

(iv) An $H_{3}$-structure $F^{0}$ on a 4-manifold $M^{0}$ with the property that $c=0$ and whose curvature mapping $K: F^{0} \rightarrow \mathcal{V}$ is a submersion onto $\Sigma_{0}^{0} \backslash\{(0,0)\}$. The symmetry group of $F^{0}$ has dimension 5 and acts transitively on $M^{0}$; however, its conformal symmetry group has dimension 6. Moreover, $F^{0}$ is the unique representative in its homothety class.

(v) The standard flat $H_{3}$-structure $F_{0}$ on $\mathbb{R}^{4}$. Its curvature mapping $K: F_{0} \rightarrow \mathcal{V}$ is just $K \equiv(0,0)$. The symmetry group of $F_{0}$ has dimension 7 and acts transitively on $\mathbb{R}^{4}$; however, its conformal symmetry group has dimension 8. Moreover, $F_{0}$ is the unique representative in its homothety class.

Thus, there exist eight homothety classes of singular torsion-free $H_{3}$-structures, the last two of which are homogeneous. Writing these structures out explicitly is somewhat tedious, even in case $(i v)$ above, the "simplest" of the non-flat $H_{3}$-structures. However, as we shall see in $\S 5$, the manifold $M^{0}$ is topologically the space of smooth plane conics in $\mathbb{R P}^{2}$ which pass through a fixed point, and its $H_{3}$-structure $F^{0}$ has a very natural geometric interpretation. 


\section{§4. Path Geometries and Exotic Holonomy}

We now turn to another approach to understanding the torsion-free $G_{3^{-}}$and $H_{3^{-}}$ structures on 4-manifolds. This approach is closely related to the theory of "path geometries" as initiated by Élie Cartan and, in a complexified form to be treated in the next section, to the twistor theory of Penrose as generalized by Hitchin [1982].

In order to begin the discussion, let us take a closer look at the $G_{3}$-representation $\mathcal{V}_{3}$. As we remarked in the introduction, $\mathcal{V}_{3}$ can be regarded as the homogeneous cubic polynomials in two indeterminates $x$ and $y$. As a result, $\mathcal{V}_{3}$ contains a $G_{3}$-invariant, 2dimensional cone $\tilde{\mathcal{C}} \subset \mathcal{V}_{3}$ which consists of the polynomials which are "perfect cubes", i.e., are of the form $(a x+b y)^{3}$ for some linear form $a x+b y \in \mathcal{V}_{1}$. Moreover, $G_{3}$ is easily characterized as the subgroup of $\operatorname{GL}\left(\mathcal{V}_{3}\right)$ which preserves $\tilde{\mathcal{C}}$. This cone can be made more familiar by noting that its projectivization $\mathcal{C} \subset \mathbb{P}\left(V_{3}\right)$ is a rational normal curve (sometimes called the "twisted cubic curve"). It is not hard to show that, for any rational normal curve $C \subset \mathbb{P}(V)$ where $V$ is a (real) vector space of dimension 4, there exists an isomorphism $\iota: V \tilde{\rightarrow} \mathcal{V}_{3}$ which identifies $C$ with $\mathcal{C}$, and that this isomorphism is unique up to composition with an element of $G_{3}$.

This leads to an alternate description of $G_{3}$-structures on 4-manifolds which will be important for our second point of view. If $F$ is a $G_{3}$-structure on $M^{4}$, then for each $x \in M$, there is a well-defined rational normal curve $C_{x} \subset \mathbb{P}\left(T_{x} M\right)$ which corresponds to $\mathcal{C}$ under any isomorphism $u: T_{x} M \stackrel{\sim}{\rightarrow} \mathcal{V}_{3}$ where $u \in F_{x}$. The "de-projectivized" cone $\tilde{C}_{x} \subset T_{x} M$ which corresponds to $\tilde{\mathcal{C}} \subset \mathcal{V}_{3}$ will be known as the "rational normal" cone (or, sometimes, the "twisted cubic" cone) of $F$ at $x$. It is easy to see that the union $C \subset \mathbb{P}(T M)$ of all of these curves is a smooth submanifold of $\mathbb{P}(T M)$ and that the natural projection $C \rightarrow M$ is a smooth submersion. Conversely, given a smooth subbundle $C \subset \mathbb{P}(T M)$ for which the fiber $C_{x}$ is a rational normal curve in $\mathbb{P}\left(T_{x} M\right)$ for each $x \in M$, there is a unique $G_{3^{-}}$ structure $F$ which corresponds to $C$ in the manner just described. Thus, a $G_{3}$-structure on $M$ may be regarded as equivalent to a smooth field of rational normal cones on $M$.

There is another geometric object in $\mathcal{V}_{3}$ which will be useful. This is the quartic cone $\tilde{\mathcal{Q}} \subset \mathcal{V}_{3}$ which is the null cone of the $H_{3}$-invariant quartic form $Q$ on $\mathcal{V}_{3}$ given by $Q(v)=\left\langle\langle v, v\rangle_{2},\langle v, v\rangle_{2}\right\rangle_{2}$. The null vectors of $Q$ on $\mathcal{V}_{3}$ are the cubic polynomials which have a double linear factor. The "conformal class" of $Q$ is preserved by $G_{3}$ and hence both the "null cone" of $Q$, i.e., $\tilde{\mathcal{Q}}$, and its projectivization $\mathcal{Q} \subset \mathbb{P}\left(\mathcal{V}_{3}\right)$ are $G_{3}$-invariant. The relationship between $\mathcal{C}$ and $\mathcal{Q}$ is simple: $\mathcal{C}$ is the singular locus of $\mathcal{Q}$ while $\mathcal{Q}$ is the tangent developable of $\mathcal{C}$. It is not hard to see that if $p \subset \tilde{\mathcal{Q}}$ is a linear 2-plane, then $p$ consists of the multiples $(a x+b y) \ell^{2}$ where $\ell \in \mathcal{V}_{1}$ is a fixed linear form defined up to a scalar multiple. Thus, the null planes of $Q$ form a 1-dimensional rational curve $\mathcal{N}$ in the Grassmannian $\operatorname{Gr}\left(2, \mathcal{V}_{3}\right)$.

Corresponding to this geometry in $\mathcal{V}_{3}$, a $G_{3}$-structure on $M$ defines a quartic null cone $\tilde{\mathcal{Q}}_{x} \subset T_{x} M$ and a rational curve $N_{x} \subset \operatorname{Gr}\left(2, T_{x} M\right)$ of null planes for each $x \in M$. We let $N$ denote the union of the $N_{x}$ as $x$ ranges over $M$ and denote the base-point projection by l: $N \rightarrow M$.

It is interesting to compare this to the more familiar case of a conformal structure of type $(2,2)$ on $M$. In that case, the space of null 2-planes at each point $x \in M$ forms two 
disconnected rational one-parameter families, the $\alpha$-planes and the $\beta$-planes in Penrose's terminology. The condition for there to exist a null surface of type $\alpha$ (respectively, of type $\beta$ ) in $M$ tangent to each $\alpha$-plane (resp. $\beta$-plane) is that the conformal structure be half conformally flat, i.e., that one of the two irreducible components of the conformal Weyl curvature vanish. Our first result will describe an analogous phenomenon for $G_{3}$-structures.

TheOREM 4.1: $A G_{3}$-structure $F$ on a four-manifold $M$ is torsion-free if and only if for every null plane $p \in N_{x}$ there exists a null surface in $M$ which passes through $x$ and for which $T_{x} S=p$.

ProOF: Let $F$ be a $G_{3}$-structure on $M$, and let $\omega, \lambda$, and $\phi$ with values in $\mathcal{V}_{3}, \mathcal{V}_{0}$, and $\mathcal{V}_{2}$ respectively denote the canonical 1 -form and intrinsic connection forms on $F$. From $\S 2$, we know that the first structure equation can be written in the form

$$
d \omega=-\lambda \wedge \omega-\langle\phi, \omega\rangle_{1}+\langle\tau, \omega\rangle_{4}
$$

where $\tau$ is a function on $F$ with values in $\mathcal{V}_{7}$ which has the equivariance

$$
R_{g}{ }^{*}(\tau)=\operatorname{det}(g)^{\frac{1}{4}} g^{-1} \cdot \tau
$$

for $g \in G_{3}$. By definition, the function $\tau$ vanishes if and only if $F$ is torsion-free.

There is a submersion $\nu: F \rightarrow N$ defined as follows. For each $u \in F_{x}$, let $\nu(u) \in N$ be the subspace of $T_{x} M$ which corresponds under $u: T_{x} M \stackrel{\sim}{\rightarrow} \mathcal{V}_{3}$ to the subspace of $\mathcal{V}_{3}$ spanned by $\left\{x^{3}, x^{2} y\right\}$. Of course, we have $\pi=l \circ \nu$. The fibers of $\nu$ are the orbits in $F$ of the subgroup $P \subset G_{3}$ which stabilizes this subspace. It will be useful in what follows to note that $P=P^{0} \cup\left(-P^{0}\right) \simeq \mathbb{Z}_{2} \times P^{0}$ where $P^{0}$ is the identity component of $P$. In particular, note that the typical fiber of $\nu$ is not connected, but consists of two components.

Let $(\mathcal{I}, \Omega)$ denote the differential ideal with independence condition on $N$ whose integrals are the canonical lifts of null surfaces. (This is just the restriction of the canonical contact system on $\operatorname{Gr}(2, T M)$ to $N \subset \operatorname{Gr}(2, T M)$.) Then $\nu$ pulls this ideal back to $F$ to be the ideal $\mathcal{I}^{\nu}$ generated by $\omega_{1}$ and $\omega_{3}$ and the independence condition $\Omega$ pulls back under $\nu$ to be represented by the 2 -form $\Omega^{\nu}=\omega_{-1} \wedge \omega_{-3}$. Thus, in order to describe the integral manifolds of $(\mathcal{I}, \Omega)$, it suffices to study the integral manifolds of $\left(\mathcal{I}^{\nu}, \Omega^{\nu}\right)$.

From the structure equation (1), we can extract the following formulas:

$$
\left.\begin{array}{rr}
d \omega_{1} \equiv 4 \phi_{2} \wedge \omega_{-1}+2160 \tau_{5} \omega_{-3} \wedge \omega_{-1} \\
d \omega_{3} \equiv \quad 5040 \tau_{7} \omega_{-3} \wedge \omega_{-1}
\end{array}\right\} \quad \text { modulo } \omega_{1}, \omega_{3} .
$$

It follows from these formulas that no integral elements of $\left(\mathcal{I}^{\nu}, \Omega^{\nu}\right)$ exist at points where $\tau_{7} \neq 0$. Of course, this implies that there are no integral elements of the original system $(\mathcal{I}, \Omega)$ at any point in $N$ of the form $p=\nu(u)$ where $\tau_{7}(u) \neq 0$. In particular, if there exists an integral manifold of $(\mathcal{I}, \Omega)$ passing through every point of $N$, then we must have $\tau_{7} \equiv 0$ on $F$. Since $\mathcal{V}_{7}$ is $G_{3}$-irreducible, this implies that we must have $\tau \equiv 0$, implying that $F$ is torsion-free. 
Conversely, suppose that $\tau \equiv 0$. Then the structure equations (2) simplify to

$$
\left.\begin{array}{l}
d \omega_{1} \equiv 4 \phi_{2} \wedge \omega_{-1} \\
d \omega_{3} \equiv \quad 0
\end{array}\right\} \quad \text { modulo } \omega_{1}, \omega_{3}
$$

Moreover, the $y^{3}$-component of $d \omega$ yields the further information that

$$
d \omega_{3} \equiv 2 \phi_{2} \wedge \omega_{1} \quad \text { modulo } \omega_{3} .
$$

It follows that the system $(\mathcal{I}, \Omega)$ has one-dimensional Cauchy characteristics and that $\mathcal{I}$ is locally equivalent to the second order contact system for curves in the plane. Thus, for each $p \in N$, there exists an integral manifold $\tilde{S}$ of $(\mathcal{I}, \Omega)$ passing through $p$. The projection of $\tilde{S}$ to $M$ yields the desired null surface $S$.

Geometrically, the Cauchy characteristics of $\mathcal{I}$ can be described as the $\mathcal{C}$-geodesics of (the intrinsic connection of) $F$. To see this, note that the Cartan system of $\mathcal{I}^{\nu}$ is generated by the system $C(\mathcal{I})=\operatorname{span}\left\{\omega_{3}, \omega_{1}, \omega_{-1}, \phi_{2}\right\}$. The connected integral manifolds of this system in $F$ project to $M$ to become the $\varphi$-geodesics whose tangent vectors at each point belong to the twisted cubic cone, hence the name $\mathcal{C}$-geodesics. In particular, note that every null surface can be regarded (locally) as a one-parameter family of $\mathcal{C}$-geodesics. The system $\mathcal{I}$ can thus be regarded as representing the conditions for selecting a oneparameter family of $\mathcal{C}$-geodesics in such a way that the resulting surface is null. Thus, the integral manifolds of $(\mathcal{I}, \Omega)$ are constructible by ODE methods alone. The main point is that the Cartan-Kähler Theorem is not needed, so our constructions do not require the assumption of real analyticity.

From now on, let us assume that $F$ is a torsion-free $G_{3}$-structure on $M$. For our purposes, the most interesting null surfaces in $M$ will be those which are totally geodesic. These surfaces are the integrals of an augmented system $\left(\mathcal{I}_{+}, \Omega\right)$ on $N$ whose pull-back under $\nu$ is the system $\left(\mathcal{I}_{+}^{\nu}, \Omega^{\nu}\right)$ where $\mathcal{I}_{+}^{\nu}$ is generated by the 1 -forms $\omega_{1}, \omega_{3}$, and $\phi_{2}$.

Proposition 4.2: If $F$ is a torsion-free $G_{3}$-structure on $M$, then the system $\mathcal{I}_{+}$is a Frobenius system of rank 3. In particular, there exists a 3-parameter family of totally geodesic null surfaces in $M$.

Proof: Since $F$ is torsion-free, we may assume that the structure equations (2.6-7) hold. Examining the $x y^{2}$ - and $y^{3}$-components of $d \omega$ and the $x^{2}$-component of $d \phi$, we get the equations

$$
d \omega_{1} \equiv d \omega_{3} \equiv d \phi_{2} \equiv 0 \quad \text { modulo } \quad \omega_{1} \omega_{3}, \phi_{2} .
$$

Of course this implies the Proposition.

REMARK: As the reader is probably aware, the only surprising aspect of this proof is that $d \phi_{2} \equiv 0$ modulo $\omega_{1} \omega_{3}, \phi_{2}$. A naïve expectation would be that the curvature term in the formula for $d \phi_{2}$ would make the formula read

$$
d \phi_{2} \equiv g \omega_{-3} \wedge \omega_{-1} \quad \text { modulo } \omega_{1} \omega_{3}, \phi_{2}
$$


for some function $g$ which is a linear combination of curvature coefficients. However, this coefficient must be zero since the "conservation of index sum" principle would say that $g$ would be assigned an index of 6 as a coefficient of some $\mathcal{V}_{k}$-valued function on $F$, while we have seen that the curvature takes values in $\mathcal{V}_{2} \oplus \mathcal{V}_{4}$, a space which has no terms of index 6.

From now on, we will refer to a connected, totally geodesic null surface of a torsion-free $G_{3}$-structure $F$ on $M$ as a sheet of $F$. The sheets of $F$ are in one-to-one correspondence with the leaves of the Frobenius system $\mathcal{I}_{+}$on $N$. Since $\mathcal{I}_{+}$is a rank 3 Pfaffian system, its space of leaves has the structure of a (not necessarily Hausdorff) three-manifold.

We shall say that $F$ is amenable if the space of leaves of $\mathcal{I}_{+}$on $N$ is Hausdorff. It can be shown that if $F$ is a torsion-free $G_{3}$-structure on $M$, and $x \in M$ is any point, then there exists an $x$-neighborhood $U \subset M$ so that the restricted $G_{3}$-structure $F_{U}$ is amenable. This is done by first showing that the canonical flat $G_{3^{-}}$structure on $\mathcal{V}_{3}$ is amenable and then showing that if $\exp _{x}: T_{x} M \rightarrow M$ is the (locally defined) exponential mapping of the connection $\varphi$, then $U=\exp _{x}(\tilde{U})$ is amenable for any sufficiently small convex neighborhood $\tilde{U}$ of $0 \in T_{x} M$. (Essentially this is true because such a $U$ can be compared "closely enough" with the flat case. Details will be left to the interested reader.) In the amenable case, we let $Y$ denote the space of sheets and let $\mathrm{r}: N \rightarrow Y$ denote the projection.

Proposition 4.3: If $F$ is an amenable, torsion-free $G_{3}$-structure on $M$, then

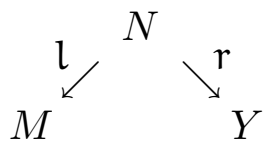

is a non-degenerate double fibration. Moreover, there exists a contact structure on $Y$ for which each of the curves $R_{x}=\mathrm{r}\left(\mathrm{l}^{-1}(x)\right)$ for $x \in M$ is a (closed) contact curve. Finally, if we let $Y^{(2)}$ denote the manifold of 2-jets of contact curves in $Y$, and let $\mathrm{r}^{(2)}: N \rightarrow Y^{(2)}$ be the mapping which sends $p \in N$ to the 2-jet of $R_{l(p)}$ at $r(p)$, then $r^{(2)}$ is a local diffeomorphism.

Before we begin the proof, note that we have the following consequences of the structure equations on $F$ :

$$
\begin{aligned}
& d \omega_{3} \equiv 2 \phi_{2} \wedge \omega_{1} \quad \text { modulo } \omega_{3} \text {, } \\
& d \omega_{1} \equiv 4 \phi_{2} \wedge \omega_{-1} \text { modulo } \omega_{3}, \omega_{1} \text {, } \\
& d \omega_{-1} \equiv 6 \phi_{2} \wedge \omega_{-3} \text { modulo } \omega_{3}, \omega_{1}, \omega_{-1} \text {, } \\
& d \omega_{-3} \equiv \quad 0 \quad \text { modulo } \omega_{3}, \omega_{1}, \omega_{-1}, \omega_{-3} \text {. }
\end{aligned}
$$

It follows from these equations that the orbits of $P^{0} \subset G_{3}$ are the Cauchy characteristic leaves of the Pfaffian system on $F$ spanned by $\left\{\omega_{3}, \omega_{1}, \omega_{-1}\right\}$. However, this system is also invariant under right action by $-1 \in G_{3}$, so this Pfaffian system is the pull-back under $\nu$ of a well defined Pfaffian system $I$ of $\operatorname{rank} 3$ on $N=F / P$. Note that the first (respectively, 
second) derived system of $I$, denoted $I^{[1]}$ (respectively, $I^{[2]}$ ), pulls back via $\nu$ to $F$ to be spanned by the form(s) $\left\{\omega_{3}, \omega_{1}\right\}$ (respectively, $\left\{\omega_{3}\right\}$ ).

Proof: First, we show that the product mapping $l \times r: N \rightarrow M \times Y$ is an immersion. By construction, the semi-basic forms for the projection $\pi=l \circ \nu: F \rightarrow M$ are spanned by the 1-forms $\left\{\omega_{-3}, \omega_{-1}, \omega_{1}, \omega_{3}\right\}$; the semi-basic forms for the projection $r \circ \nu: F \rightarrow Y$ are spanned by the 1 -forms $\left\{\phi_{2}, \omega_{1}, \omega_{3}\right\}$; and the semi-basic forms for the projection $\nu: F \rightarrow N$ are spanned by the forms $\left\{\omega_{-3}, \omega_{-1}, \phi_{2}, \omega_{1}, \omega_{3}\right\}$. Since this latter collection is contained in the union of the first two, it follows that $l \times r$ is an immersion. (Note that we have not proved that $l \times r$ is an embedding, which is the usual double fibration axiom. This stronger statement may well fail.)

Now, the intersection of the first two spans is the Pfaffian system spanned by the forms $\left\{\omega_{1}, \omega_{3}\right\}$. By the first two of the equations (6), it follows that this system does not contain any Frobenius sub-system. Thus, the double fibration is non-degenerate.

Now the first equation of (6) shows that the Cartan system of $I^{[2]}$ is a rank 3 Pfaffian system which consists of the semi-basic forms for the projection $\mathrm{r}: N \rightarrow Y$. Since, by construction, the fibers of $r$ are connected, a standard result (see $\mathrm{BCG}^{3}[\mathbf{1 9 9 0}]$ ), then implies that there exists a rank 1 Pfaffian system $\left(I^{[2]}\right)^{b}$ on $Y$ which pulls back to $N$ via $r$ to become the Pfaffian system $I^{[2]}$. The first equation of $(6)$ then implies that $\left(I^{[2]}\right)^{b}$ defines a contact structure on $Y$. We will denote by $L \subset T^{*} Y$ the line bundle whose space of sections is equal to $\left(I^{[2]}\right)^{b}$.

Now for each $x \in M$, the curve $R_{x}$ is a contact curve for this contact structure (i.e., is an integral curve of $\left.\left(I^{[2]}\right)^{b}\right)$. To see this, note that the fiber $N_{x}=l^{-1}(x) \subset N$ is a smooth, connected, compact curve and its inverse image under $\nu$ is $\pi^{-1}(x)=F_{x}$. Since $\omega$ clearly vanishes when pulled back to $F_{x}$, it follows that $N_{x}$ is an integral curve of $I$ and hence that $R_{x}=\mathrm{r}\left(N_{x}\right)$ is an integral curve of $L$.

Let $\sigma_{2}: Y^{(2)} \rightarrow Y$ be the 5 -dimensional bundle of 2 -jets of contact curves in $Y$ and let $L^{(2)} \subset T^{*} Y^{(2)}$ be the canonical contact Pfaffian system. By a standard result in the theory of Pfaffian systems, namely the Goursat Normal Form Theorem (see BCG ${ }^{3}[\mathbf{1 9 9 0}]$, particularly Chapter II), the structure equations (6) imply that there is a unique mapping $\mathrm{r}^{\prime}: N \rightarrow Y^{(2)}$ which satisfies $\mathrm{r}=\sigma_{2} \circ \mathrm{r}^{\prime}$ and which pulls $L^{(2)}$ back to be $I$. The map $\mathrm{r}^{\prime}$ can be defined as follows: Let $p \in N$ be any point, and let $\gamma \subset N$ be any integral curve of $I$ which passes through $p$ and is transverse to the fibers of $r$. Then $\mathrm{r}^{\prime}(p)$ is defined to be the 2-jet of the curve $r(\gamma)$ at $r(p)$. (Although we have invoked the Goursat Theorem, the well-definition of this mapping can be checked directly by the interested reader. See the discussion below on ODE.) It follows from the general theory that $r^{\prime}$ is a local diffeomorphism. By construction, the fiber of $l$ through any $p \in N$ is an integral curve of $I$, and hence $r^{\prime}$ is equal to $r^{(2)}$ as defined in the proposition.

It is worth remarking that, in fact, $L$ is the unique contact structure on $Y$ with respect to which each of the curves $R_{x}$ is a contact curve. This follows since our discussion has shown that the curves of the form $R_{x}$ which pass through a given point $y \in Y$ serve to "fill out" an open set in the space of 2-jets of contact curves passing through $y$. Thus, there is only one 2-plane $E_{y} \subset T_{y} Y$ which contains all of the tangent vectors to these curves, and hence its annihilator $E_{y}^{\perp} \subset T_{y}^{*} Y$ is unique and must be $L_{y}$. 
We have shown how to associate to each torsion-free amenable $G_{3}$-structure $F$ a contact 3-manifold endowed with a "non-degenerate" 4-parameter family of contact curves. It is instructive to see what this data looks like in local coordinates. The coordinate form of the Goursat Normal Form Theorem asserts that every point $p \in N$ has a neighborhood $U$ on which there exist local coordinates $\psi=\left(x, y^{0}, y^{1}, y^{2}, y^{3}\right)$ so that if we define $\theta^{i}=$ $d y^{i}-y^{i+1} d x$ for $i=0,1,2$, then $I^{[2]}$ is spanned by $\theta^{0}$ while $I^{[1]}$ is spanned by $\left\{\theta^{0}, \theta^{1}\right\}$ and $I^{[0]}=I$ is spanned by $\left\{\theta^{0}, \theta^{1}, \theta^{2}\right\}$. The fibration $r$ can then be represented locally in these coordinates by $\left(x, y^{0}, y^{1}, y^{2}, y^{3}\right) \mapsto\left(x, y^{0}, y^{1}\right)$. Moreover, after possibly shrinking $U$, the coordinate system can be chosen so that $x$ restricts to each fiber of $l$ to become a local coordinate. It then follows that the l-fibers are the integral curves of the rank 4 Pfaffian system spanned by the 1 -forms $\left\{\theta^{0}, \theta^{1}, \theta^{2}, d y^{3}-\Phi \circ \psi d x\right\}$ where $\Phi$ is some smooth function on $\psi(U) \subset \mathbb{R}^{5}$.

It follows that the (smooth) integral curves of $I$ on which $d x$ is non-zero can be written locally in the form $y^{i}=f^{(i)}(x)$ for some (smooth) function $f$, and that the fibers of $l$ then correspond to the functions $f$ which satisfy the fourth order ODE

$$
f^{(4)}=\Phi\left(x, f, f^{\prime}, f^{\prime \prime}, f^{\prime \prime \prime}\right) \text {. }
$$

From this point of view, it is clear that the set of curves of the form $R_{x}$ which pass through a given point $s \in Y$ is a two-parameter family which "fills out" an open set in the set of 2-jets of contact curves passing through $s$.

Thus, our discussion so far has shown how to associate a fourth order oDE to any amenable torsion-free $G_{3}$-structure. This association is almost canonical. The only noncanonical aspect of our construction is the choice of local contact coordinates on $N$. However, by the usual prolongation procedure, these coordinates are determined by first three of these coordinates, namely $\left(x, y^{0}, y^{1}\right)$ which are local coordinates in $Y$. Thus, the odE (7) is uniquely determined up to an action of the contact pseudo-group in dimension 3. In the classical language (compare Cartan [1941] and Chern [1940]), one says that the equation (7) is determined up to contact equivalence.

For the remainder of this section, we shall discuss the feasibility of reversing this procedure. In particular, we shall show that it is possible to reconstruct (at least locally) the original torsion-free $G_{3}$-structure from the data of the fourth order equation. Moreover we shall determine which (contact equivalence classes of) fourth order equations arise from torsion-free $G_{3}$-structures by our construction.

This process may be viewed as part of the program of "geometrizing" ordinary differential equations, as proposed by Cartan [1938]. We refer the interested reader to the works of Chern and Cartan listed in the references for further information about the "geometrization" of lower order ODE.

In order to formulate this reconstruction problem more precisely, we introduce a few notions from the theory of pseudo-groups. In $\mathbb{R}^{5}$ with coordinates $\left(x, y^{0}, y^{1}, y^{2}, y^{3}\right)$, we introduce the pseudo-group $\Gamma \subset \operatorname{Diff}_{\text {loc }}\left(\mathbb{R}^{5}\right)$ which consists of the local diffeomorphisms of $\mathbb{R}^{5}$ which preserve the rank 3 Pfaffian system $I_{0}$ generated by the three 1 -forms

$$
\theta^{0}=d y^{0}-y^{1} d x, \quad \theta^{1}=d y^{1}-y^{2} d x, \quad \theta^{2}=d y^{2}-y^{3} d x .
$$


Given a 5-manifold $Z$, a $\Gamma$-structure on $Z$ is, by definition, a maximal atlas $A$ of coordinate charts whose transition diffeomorphisms lie in $\Gamma$. Associated to such a $\Gamma$ structure on $Z$, there is a well-defined rank 3 Pfaffian system $I$ which is mapped onto $I_{0}$ by any local coordinate chart in $A$. Of course, the system $I$ in turn serves to define the $\Gamma$-atlas $A$ as the set of coordinate charts on $Z$ which pull-back $I_{0}$ to $I$, so we may regard the specification of $I$ as equivalent to the specification of $A$.

Since $\Gamma$ also preserves the derived systems $I_{0}^{[1]}$ and $I_{0}^{[2]}$ spanned by $\left\{\theta^{0}, \theta^{1}\right\}$ and $\left\{\theta^{0}\right\}$ respectively, it follows that these also correspond to well-defined systems $I^{[1]}$ and $I^{[2]}$ on $Z$ of ranks 2 and 1 respectively. Moreover, $\Gamma$ preserves the Cauchy foliations of $I_{0}^{[1]}$ and $I_{0}^{[2]}$ and hence there exist well-defined foliations of codimensions 4 and 3 respectively on any $Z^{5}$ endowed with a $\Gamma$-structure. For our purposes, the Cauchy foliation of $I^{[2]}$ will be the most important. This is the foliation which maps under any coordinate chart in $A$ to the simultaneous level sets of the functions $x, y^{0}$, and $y^{1}$. Generalizing our previous case of $\mathcal{I}_{+}$on $N$, we shall say that a $\Gamma$-structure on $Z$ is amenable if the space $Y^{3}$ of Cauchy leaves of $I^{[2]}$ is Hausdorff.

An integral curve $\gamma$ of $I$ is said to be admissible if it is transverse to the Cauchy leaves of $I^{[2]}$. In an A-chart, this means that, when $\gamma$ is written in the form $\gamma(t)=$ $\left(\left(x(t), y^{0}(t), y^{1}(t), y^{2}(t), y^{3}(t)\right)\right.$, the "reduced" curve $\bar{\gamma}(t)=\left(\left(x(t), y^{0}(t), y^{1}(t)\right)\right.$ is also an immersed curve.

As an example, note that the contact system $I_{0}$ on the third order jet space $J^{3}(\mathbb{R}, \mathbb{R})$ endows $J^{3}(\mathbb{R}, \mathbb{R})$ with a canonical $\Gamma$-structure and the solution curves of a fourth order ODE describe a foliation of $J^{3}(\mathbb{R}, \mathbb{R})$ by admissible integral curves of $I_{0}$. As another example, the one that concerns us most in this paper, note that the manifold $N$ constructed from any torsion-free $G_{3}$-structure possesses a natural $\Gamma$-structure defined by the Pfaffian system $I$ as well as a foliation by admissible integral curves of $I$.

In the case where $Z$ has an amenable $\Gamma$-structure, a foliation of $Z$ by admissible integral curves of $I$ has a natural interpretation as a four-parameter family of contact curves in the space $Y$. This latter family of curves determines a contact path geometry on $Y$ in Cartan's sense. We do not want to limit ourselves to the amenable case, so we will refer to a 5 -manifold $Z$ endowed with a $\Gamma$-structure and a foliation by admissible integral curves as a generalized contact path geometry.

In the remainder of this section, we will describe some of the local invariants of a generalized contact path geometry. This description will be applied in the next section to relate a certain "twistor space" to the geometry of torsion-free $G_{3}$-structures.

Let $G \subset \mathrm{GL}(5, \mathbb{R})$ denote the subgroup which consists of Jacobian matrices of elements of $\Gamma$ relative to the coframe

$$
\underline{\vartheta}=\left(\begin{array}{c}
\underline{\vartheta}^{0} \\
\underline{\vartheta}^{1} \\
\underline{\vartheta}^{2} \\
\underline{\vartheta}^{3} \\
\underline{\vartheta}^{4}
\end{array}\right)=\left(\begin{array}{c}
6 \theta^{0} \\
6 \theta^{1} \\
3 \theta^{2} \\
d y^{3} \\
\frac{1}{2} d x
\end{array}\right) .
$$


(The choice of constants is cosmetic, but it does help us compare the structure equations we are about to derive with the ones we have already derived.) It can be shown that the Lie algebra of $G$ is the subalgebra $\mathfrak{g} \subset \mathfrak{g l}(5, \mathbb{R})$ consisting of those matrices of the form

$$
\left(\begin{array}{ccccc}
m+3 f_{0} & 0 & 0 & 0 & 0 \\
a_{1}+6 f_{1} & m+f_{0} & 0 & 0 & 0 \\
a_{2}+2 a_{4} & a_{1}+4 f_{1} & m-f_{0} & 0 & 0 \\
a_{3} & a_{2}+a_{4} & a_{1}+2 f_{1} & m-3 f_{0} & a_{0} \\
a_{6} & a_{5} & 0 & 0 & 2 f_{0}
\end{array}\right)
$$

where $m, f_{0}, f_{1}$, and $a_{i}$ for $0 \leq i \leq 6$ are arbitrary real numbers. Moreover, $G$ consists of two connected components: the identity component $G^{o} \subset \mathrm{GL}(5, \mathbb{R})$, which is a closed subgroup, and the coset $h G^{o}$ where $h=\operatorname{diag}(-1,-1,-1,-1,1)$. (The choice of basis for $\mathfrak{g}$ implicit in the entry labeling in (9) is also partly for cosmetic reasons and partly for ease of reference in an argument to be presented below.)

It follows that, associated to every $\Gamma$-structure $A$ on $Z^{5}$, there is a unique (first order) $G$-structure $F \subset \mathcal{F}$ (where $\mathcal{F}$ is the $\mathbb{R}^{5}$-coframe bundle of $Z$ ) with the property that $\phi^{*}(\underline{\vartheta}$ ) is a section of $F$ over $U \subset Z$ whenever $\phi: U \rightarrow \mathbb{R}^{5}$ is a local coordinate system belonging to $A$. Of course, the $G$-structure $F$ in turn determines the $\Gamma$-structure $A$, so these may be regarded as equivalent.

We define a closed subgroup $P \subset G$ by the condition that $P=P^{o} \cup h P^{o}$ where $P^{o}$ is the connected Lie sub-group of $G$ whose Lie algebra $\mathfrak{p} \subset \mathfrak{g}$ consists of the matrices of the form (9) where all of the $a_{i}$ have been set to zero.

Proposition 4.4: Let $Z$ be a 5-manifold endowed with a $\Gamma$-structure and let $F$ be the corresponding $G$-structure. Let $\mathcal{L}$ be a foliation of $Z$ by admissible integral curves of $I$. Then there exists a unique $P$-structure $F_{\mathcal{L}} \subset F$ which has the following properties:

(i) If $\sigma: U \rightarrow F_{\mathcal{L}}$ is any local section where $U \subset Z$ is open, then $\sigma=\left(\sigma^{0}, \sigma^{1}, \ldots, \sigma^{4}\right)$ has the property that the leaves of $\mathcal{L} \cap U$ are integral curves of the forms $\sigma^{0}, \sigma^{1}, \sigma^{2}, \sigma^{3}$.

(ii) The structure equations of $F_{\mathcal{L}}$ take the form

$$
\left(\begin{array}{c}
d \vartheta^{0} \\
d \vartheta^{1} \\
d \vartheta^{2} \\
d \vartheta^{3} \\
d \vartheta^{4}
\end{array}\right)=-\left(\begin{array}{ccccc}
\lambda+3 \kappa_{0} & -2 \vartheta^{4} & 0 & 0 & 0 \\
6 \kappa_{1} & \lambda+\kappa_{0} & -4 \vartheta^{4} & 0 & 0 \\
0 & 4 \kappa_{1} & \lambda-\kappa_{0} & -6 \vartheta^{4} & 0 \\
0 & 0 & 2 \kappa_{1} & \lambda-3 \kappa_{0} & 0 \\
0 & 0 & 0 & 0 & 2 \kappa_{0}
\end{array}\right) \wedge\left(\begin{array}{c}
\vartheta^{0} \\
\vartheta^{1} \\
\vartheta^{2} \\
\vartheta^{3} \\
\vartheta^{4}
\end{array}\right)+\left(\begin{array}{c}
0 \\
0 \\
T^{2} \\
T^{3} \\
T^{4}
\end{array}\right)
$$

for some 1 -forms $\kappa_{0}, \kappa_{1}$, and $\lambda$, and where the $T^{i}$ are 2-form expressions in the $\vartheta^{i}$ which satisfy

$$
\begin{aligned}
& T^{2}=2 A \vartheta^{4} \wedge \vartheta^{0} \\
& T^{3}=\vartheta^{4} \wedge\left(A \vartheta^{1}+B \vartheta^{0}\right)+\vartheta^{0} \wedge\left(C \vartheta^{2}+D \vartheta^{1}\right) \\
& T^{4} \equiv \quad 0 \quad \text { modulo } \vartheta^{0}, \vartheta^{1}
\end{aligned}
$$

for some functions $A, B, C$, and $D$ on $F_{\mathcal{L}}$. 
Moreover, the 1-forms $\kappa_{0}, \kappa_{1}$, and $\lambda$ and the functions $A, B, C$, and $D$ which make these structure equations hold are unique.

Proof: The proof is a calculation which is a little long but which offers no difficulty (particularly to MAPLE) and we will only give an outline here.

First, restrict to the sub-bundle $F_{0}$ of $F$ which satisfies $(i)$. The sub-bundle $F_{0}$ is a principal bundle for the subgroup $G_{0} \subset G$ whose Lie algebra $\mathfrak{g}_{0}$ is the set of matrices of the form (9) for which $a_{0}=0$ and which satisfies $G_{0}=G_{0}^{o} \cup h G_{0}^{o}$ where $G_{0}^{o}$ is the identity component of $G_{0}$. The structure reduction by Cartan's method of equivalence then proceeds in three stages:

The intrinsic torsion of the $G_{0}$-structure $F_{0}$ takes values in a single two dimensional $G_{0}$-orbit in the Spencer cohomology group $H^{0,2}\left(\mathfrak{g}_{0}\right)$. There is a point $\tau_{0}$ on this orbit whose stabilizer $G_{1} \subset G_{0}$ is the subgroup whose Lie algebra $\mathfrak{g}_{1}$ is the set of matrices of the form (9) for which $a_{0}=a_{1}=a_{2}=0$ and which satisfies $G_{1}=G_{1}^{o} \cup h G_{1}^{o}$ where $G_{1}^{o}$ is the identity component of $G_{1}$. We define $F_{1} \subset F_{0}$ to be the inverse image of $\tau_{0}$ under the intrinsic torsion map of $F_{0}$. Then $F_{1}$ is a principal $G_{1}$-bundle.

The intrinsic torsion of the $G_{1}$-structure $F_{1}$ takes values in a single three dimensional $G_{1}$-orbit in the Spencer cohomology group $H^{0,2}\left(\mathfrak{g}_{1}\right)$. There is a point $\tau_{1}$ on this orbit whose stabilizer $G_{2} \subset G_{1}$ is the subgroup whose Lie algebra $\mathfrak{g}_{2}$ is the set of matrices of the form (9) for which $a_{0}=a_{1}=a_{2}=a_{3}=a_{4}=a_{5}=0$ and which satisfies $G_{2}=G_{2}^{o} \cup h G_{2}^{o}$ where $G_{2}^{o}$ is the identity component of $G_{2}$. We define $F_{2} \subset F_{1}$ to be the inverse image of $\tau_{1}$ under the intrinsic torsion map of $F_{1}$. Then $F_{2}$ is a principal $G_{2}$-bundle.

The intrinsic torsion of the $G_{2}$-structure $F_{2}$ need not take values in a single $G_{2}$-orbit in $H^{0,2}\left(\mathfrak{g}_{2}\right)$. However, there is a transitive affine action of $G_{2}$ on $\mathbb{R}$ and an affine mapping $H^{0,2}\left(\mathfrak{g}_{2}\right) \rightarrow \mathbb{R}$ which is $G_{2}$-equivariant and for which the stabilizer of $0 \in \mathbb{R}$ is the subgroup $P \subset G_{2}$ as defined above. Thus, we set $F_{\mathcal{L}}$ equal to the inverse image of $0 \in \mathbb{R}$ under the reduced intrinsic torsion mapping of $F_{2}$.

Since, as is easily computed, $\mathfrak{p}^{(1)}=0$, the connection described in the statement of the Proposition is unique. Further details, including that the torsion has the stated form, will be left to the reader.

The reader may wonder why we have stopped the reduction at the third stage. The reason is that, while all of the reductions so far have been ageneric, i.e., accomplished without using any genericity assumptions, any further reductions would require some sort of genericity hypotheses. (In the terminology of Gardner [1989], one says that the method of equivalence "branches" at this point.)

To see why, note that the case where all of the $T^{i}$ vanish does occur: The flat $G_{3^{-}}$ structure on $\mathbb{R}^{4}$ gives rise to a generalized path geometry on $N=\mathbb{R}^{4} \times \mathbb{R} \mathbb{P}^{1}$ in which all of the $T^{i}$ vanish. In this case, there is no canonical reduction of this $F_{\mathcal{L}}$, for in fact, the automorphism group of the flat $G_{3}$-structure acts transitively on $F_{\mathcal{L}}$.

Any reduction of $F_{\mathcal{L}}$ in the general case must therefore be based on some genericity assumption such as $A \neq 0$. We do not wish to make genericity assumptions since they are awkward for the twistor theory in the next section. Fortunately, for our purposes, genericity assumptions will not be needed anyway. 
Now, using the structure equations in Proposition 4.4, it is easy to show that the semibasic symmetric differential forms $A\left(\vartheta^{4}\right)^{3}$ and $C \vartheta^{4} \circ \vartheta^{0}$ are $P$-invariant and therefore there exist a well defined symmetric cubic form $\mathcal{A}$ and a well defined symmetric quadratic form $\mathcal{C}$ on $Z$ which pull-back to $F_{\mathcal{L}}$ to become $A\left(\vartheta^{4}\right)^{3}$ and $C \vartheta^{4} \circ \vartheta^{0}$ respectively. We shall refer to $\mathcal{A}$ and $\mathcal{C}$ as primary invariants of the foliation $\mathcal{L}$.

If $\mathcal{A}$ vanishes identically, then the semi-basic quartic differential form $B\left(\vartheta^{4}\right)^{4}$ is $P$ invariant and therefore there exists a well defined symmetric quartic form $\mathcal{B}$ on $Z$ which pulls-back to $F_{\mathcal{L}}$ to become $B\left(\vartheta^{4}\right)^{4}$. Similarly, if $\mathcal{C}$ vanishes, then $D \vartheta^{4} \circ\left(\vartheta^{0}\right)^{2}$ is $P$-invariant and therefore there exists a well defined symmetric cubic form $\mathcal{D}$ on $Z$ which pulls-back to $F_{\mathcal{L}}$ to become $D \vartheta^{4} \circ\left(\vartheta^{0}\right)^{2}$. We shall refer to $\mathcal{B}$ and $\mathcal{D}$ (when either exists) as secondary invariants of the foliation $\mathcal{L}$.

For the reader who is comparing our treatment with the theory of contact equivalence of second (respectively, third) order ODE as developed in Cartan [1938] (respectively, Chern [1940]), the functions $A$ and $C$ represent what the classical papers call "relative invariants" and are analogous to the Wünschmann invariant encountered in the third order theory. In more modern language, "relative invariants" are just sections of certain natural line bundles associated with the geometry. As we shall see below, the "line bundle" interpretation is a useful viewpoint.

The invariants $A, B, C$, and $D$ can be written out explicitly for the generalized contact path geometry defined by the equations (7) as polynomials in the derivatives of $\Phi$ of order less than or equal to three. However, the explicit expressions are quite complicated. It is useless to write them out here.

Theorem 4.5: Let $Z$ be a 5-manifold with $F$ and $\mathcal{L}$ as in Proposition 4.4. The primary and secondary invariants of the foliation $\mathcal{L}$ vanish if and only if the $P$-structure $F_{\mathcal{L}}$ on $Z$ is locally equivalent to the $P$-structure $F$ on $N$ associated to a torsion-free $G_{3}$-structure on some 4-manifold $M$.

In order to make sense of this theorem, it will be necessary to first explain how the group $P$ is being identified in the two cases. In our original definition of $P$, it was the subgroup of $G_{3}=\mathrm{GL}(2, \mathbb{R})$ which preserved the subspace $W_{3} \subset \mathcal{V}_{3}$ spanned by $\left\{x^{3}, x^{2} y\right\}$. Of course, this subgroup also acts on $\mathcal{V}_{2}$, and it is easy to see that it preserves the subspace $W_{2} \subset \mathcal{V}_{2}$ spanned by $\left\{x^{2}, x y\right\}$. If we identify $\mathbb{R}^{5}$ with the vector space $\mathcal{V}_{3} \oplus\left(\mathcal{V}_{2} / W_{2}\right)$ by using the basis $\left(x^{3}, x^{2} y, x y^{2}, y^{3}, y^{2} \bmod W_{2}\right)$, then $P$ acts on $\mathbb{R}^{5}$. It is easy to see that embedding $P$ into $\mathrm{GL}(5, \mathbb{R})$ this way yields the subgroup of $G$ that we defined in the course of our structure reduction in the proof of Proposition 4.4.

ProOF: First, let us start with a torsion-free $G_{3}$-structure on $M$ and construct the bundle $F$ together with its connection forms $\omega, \lambda$, and $\phi$. The first structure equation is $d \omega=$ $-\lambda \wedge \omega-\langle\phi, \omega\rangle_{1}$ and can be written explicitly in the form

$$
\left(\begin{array}{l}
d \omega_{3} \\
d \omega_{1} \\
d \omega_{-1} \\
d \omega_{-3}
\end{array}\right)=-\left(\begin{array}{cccc}
\lambda+3 \phi_{0} & -2 \phi_{2} & 0 & 0 \\
6 \phi_{-2} & \lambda+\phi_{0} & -4 \phi_{2} & 0 \\
0 & 4 \phi_{-2} & \lambda-\phi_{0} & -6 \phi_{2} \\
0 & 0 & 2 \phi_{-2} & \lambda-3 \phi_{0}
\end{array}\right) \wedge\left(\begin{array}{l}
\omega_{3} \\
\omega_{1} \\
\omega_{-1} \\
\omega_{-3}
\end{array}\right)
$$


while the $y^{2}$-component of the second structure equation implies that

$$
d \phi_{2} \equiv-2 \phi_{0} \wedge \phi_{2} \quad \text { modulo } \quad \omega_{3}, \omega_{1} \text {. }
$$

By considering the coframing correspondence

$$
\left(\omega_{3}, \omega_{1}, \omega_{-1}, \omega_{-3}, \phi_{2}, \phi_{0}, \phi_{-2}, \lambda\right) \longleftrightarrow\left(\vartheta^{0}, \vartheta^{1}, \vartheta^{2}, \vartheta^{3}, \vartheta^{4}, \kappa_{0}, \kappa_{1}, \lambda\right)
$$

it becomes clear that the bundle $\nu: F \rightarrow N$ is the $P$-reduction $F_{\mathcal{L}}$ of the $G$-structure over $N$ defined by the system $I$ relative to the foliation $\mathcal{L}$ of $N$ which consists of the fibers of l: $N \rightarrow M$. Moreover, the structure equations on $F$ clearly imply that the primary and secondary invariants vanish for this $P$-structure on $N$. This establishes the theorem in one direction.

To go in the other direction, let us suppose that the primary and secondary invariants of $\mathcal{L}$ vanish. Then on $F_{\mathcal{L}}$, we have $T^{2}=T^{3}=0$. It follows that the forms

$$
\omega=\vartheta^{3} x^{3}+\vartheta^{2} x^{2} y+\vartheta^{1} x y^{2}+\vartheta^{0} y^{3}
$$

and

$$
\phi=\kappa_{1} x^{2}+\kappa_{0} x y+\vartheta^{4} y^{2}
$$

together with $\lambda$ satisfy the first structure equation for a torsion-free $G_{3}$-structure. It is now straightforward to show that, if $U \subset Z$ is an open subset on which the foliation $\mathcal{L}$ is amenable, then there is an induced torsion- free $G_{3}$-structure $F^{*}$ on the leaf space $M^{*}=U / \mathcal{L}$ and that there is a canonical immersion of $F_{\mathcal{L}}$ restricted to $U$ into $F^{*}$ which is a local diffeomorphism and which pulls the canonical forms on $F^{*}$ back to $F_{\mathcal{L}}$ so that the correspondence (12) becomes an identity. Further details will be left to the reader.

\section{$\S 5$. Twistor Theory and Exotic Holonomy}

In view of Theorem 4.5, one method of constructing torsion-free $G_{3}$-structures is to construct a generalized contact path geometry whose primary and secondary invariants vanish. Doing this directly is not easy. In terms of the fourth order oDE defined by (4.7), these conditions are equivalent to a (non-involutive) system of non-linear, third order PDE for the function $\Phi$. It is not even clear that there are any non-trivial solutions.

However, for the same geometric problem in the holomorphic category, there is another approach, based on the Kodaira deformation theory of complex manifolds and submanifolds, which is suggested by Penrose's non-linear graviton construction and its generalization by LeBrun $[\mathbf{1 9 8 3}]$ and Hitchin $[\mathbf{1 9 8 2}]$. Basically, the idea of this theory is to consider the deformation space of a rational curve (i.e., a compact Riemann surface of genus zero) in a complex manifold whose normal bundle is a sum of non-negative line bundles. This deformation space is a smooth manifold consisting of the "nearby" rational curves and it inherits a "geometry" whose properties depend on the Grothendieck type of the normal bundle of the original rational curve. 
We shall not attempt a recounting of the full theory, but instead refer the reader to Hitchin's paper. Also, we shall not always give full details in the argument below since they are generally quite analogous to those in the examples treated in that paper.

Our motivation for moving into the holomorphic setting can be explained as follows: If $F$ is a real-analytic torsion-free $G_{3}$-structure on a real 4 -manifold $M$, then, at least locally, $M$ can be embedded as a real slice of a complex 4-manifold $\mathcal{M}$ and $F$ can be extended to a $\mathcal{G}_{3}$-structure $\mathcal{F}$ where $\mathcal{G}_{3}$ is the connected holomorphic Lie subgroup of $\operatorname{Aut}\left(\mathcal{V}_{3} \otimes \mathbb{C}\right)$ whose Lie algebra is the complexification of $\mathfrak{g}_{3}$. The structure equations of this "holomorphicized" structure are analogous to those in the real category in every way. In particular, in the amenable case (which can always be arranged locally by passing to a suitably small neighborhood), the holomorphic version of Proposition 4.3 holds where the double fibration (4.5) is replaced by a "complexified" version in which the general fiber of the mapping $l$ is a copy of $\mathbb{P}^{1}$. The sheet space $\mathcal{Y}$ then becomes a complex contact 3 -fold and the corresponding complexified curves $\mathcal{R}_{x}$ are rational contact curves in the complex contact 3 -fold $\mathcal{Y}$.

Thus, the points of $\mathcal{M}$ can be thought of as rational contact curves in $\mathcal{Y}$. It is not true that $\mathcal{M}$ constitutes the complete deformation space of "nearby" rational curves, as in the cases treated previously by Penrose and Hitchin, but, as we shall see, it does constitute the complete deformation space of "nearby" rational contact curves.

We will need some information about the deformations of an unramified rational contact curve in a general complex contact 3 -fold. For the sake of avoiding any possible confusion, let us note that, by definition, an unramified rational curve in a complex manifold $\mathcal{Y}$ is an equivalence class of holomorphic immersions $\phi: \mathbb{P}^{1} \rightarrow \mathcal{Y}$ where the equivalence relation is reparametrization in the domain, i.e., $\phi_{1} \sim \phi_{2}$ iff $\phi_{1}=\phi_{2} \circ \psi$ where $\psi: \mathbb{P}^{1} \rightarrow \mathbb{P}^{1}$ is a linear fractional transformation. It is well-known that such an equivalence class is determined completely by its image $C \subset \mathcal{Y}$, so, by abuse of language, we shall speak of $C$ as a rational curve. To say that $C$ is embedded means that $C$ is the image of a one-to-one holomorphic immersion $\phi: \mathbb{P}^{1} \rightarrow \mathcal{Y}$.

The following proposition is derivable by standard "twistorial" techniques, but we give a more deformation-theoretic proof since we will need the information that this proof provides about local coordinates in the moduli space.

Proposition 5.1: Let $\mathcal{Y}$ denote a complex contact 3-fold where $L \subset T^{*} \mathcal{Y}$ denotes the holomorphic line bundle which defines the contact structure on $\mathcal{Y}$. Suppose that $C \subset \mathcal{Y}$ is an unramified rational contact curve in $\mathcal{Y}$ and that the restriction of $L$ to $C$ is isomorphic to $\mathcal{O}(-k-1)$ for some integer $k \geq 0$. Then then the normal bundle of $C$ in $\mathcal{Y}$ is isomorphic to $\mathcal{O}(k) \oplus \mathcal{O}(k)$. In particular, the moduli space $\mathcal{Z}$ of unramified rational curves in $\mathcal{Y}$ is smooth and of complex dimension $2 k+2$ near $C$. Moreover, the subspace $\mathcal{M}_{k} \subset \mathcal{Z}$ which consists of contact curves to which the contact bundle $L$ restricts to be isomorphic to $\mathcal{O}(-k-1)$ is a smooth submanifold of $\mathcal{Z}$ of complex dimension $k+2$.

PROOF: First, we will recall a few facts about complex contact geometry.

If $L \subset T^{*} \mathcal{Y}$ is the line bundle determining the contact structure, then $L^{2} \simeq K_{\mathcal{Y}}$. To see this, note that the mapping $D: \mathcal{O}(L) \rightarrow \mathcal{O}(K \mathcal{Y})$ defined by $D(\theta)=\theta \wedge d \theta$ for any local 
holomorphic section $\theta$ of $L$ satisfies $D(f \theta)=f^{2} D(\theta)$ for any local holomorphic function $f$ and thus induces a well-defined sheaf mapping $\mathcal{D}: L^{2} \rightarrow K_{\mathcal{Y}}$. This is an isomorphism since the hypothesis that $L$ defines a contact structure on $\mathcal{Y}$ implies that $D(\theta)$ is non-zero wherever $\theta$ is non-zero. Now, if $L^{\perp} \subset T \mathcal{Y}$ is the rank two vector bundle annihilated by the sections of $L$, then standard arguments combined with the above isomorphism imply that $\Lambda^{2}\left(L^{\perp}\right) \simeq L^{*}$. Note also that we have a canonical short exact sequence of vector bundles

$$
0 \longrightarrow L^{\perp} \longrightarrow T \longrightarrow L^{*} \longrightarrow 0
$$

where $T$ is the holomorphic tangent bundle of $\mathcal{Y}$.

Now, by hypothesis, $C \simeq \mathbb{P}^{1}$ is unramified, so the natural sequence

$$
0 \longrightarrow \tau \longrightarrow T_{\mid C} \longrightarrow N_{C} \longrightarrow 0
$$

is exact where $\tau \simeq \mathcal{O}(2)$ is the tangent bundle of $C$ and $N_{C}$ is the normal bundle of the immersion. (We caution the reader that, in algebraic geometry, the normal bundle is often defined as a certain sheaf theoretic quotient. This alternate definition can disagree with $N_{C}$ as we have defined it if $C$ is not embedded.) Moreover, since $C$ is a contact curve, we have an exact inclusion $0 \rightarrow \tau \rightarrow L_{\mid C}^{\perp}$. It follows that

$$
\tau \otimes\left(L_{\mid C}^{\perp} / \tau\right) \simeq \Lambda^{2}\left(L_{\mid C}^{\perp}\right) \simeq L_{\mid C}^{*} \simeq \mathcal{O}(k+1) .
$$

Thus, we must have $L_{\mid C}^{\perp} / \tau \simeq \tau^{*} \otimes \mathcal{O}(k+1) \simeq \mathcal{O}(k-1)$. From (1) we get the exactness of the sequence

$$
0 \longrightarrow L_{\mid C}^{\perp} / \tau \longrightarrow N_{C} \longrightarrow L_{\mid C}^{*} \longrightarrow 0
$$

and this implies that the normal bundle of $C$ fits into an exact sequence of the form

$$
0 \longrightarrow \mathcal{O}(k-1) \longrightarrow N_{C} \longrightarrow \mathcal{O}(k+1) \longrightarrow 0
$$

By standard arguments, it then follows that $N_{C}$ is isomorphic to either $\mathcal{O}(k-1) \oplus \mathcal{O}(k+1)$ or $\mathcal{O}(k) \oplus \mathcal{O}(k)$ according to whether the exact sequence (2) does or does not split. In either case, since $k \geq 0$ by hypothesis, we have

$$
\begin{aligned}
& h^{0}\left(N_{C}\right)=2 k+2, \\
& h^{1}\left(N_{C}\right)=0 .
\end{aligned}
$$

If $C$ is embedded, then Kodaira's Main Theorem in Kodaira [1962], hereinafter referred to as KMT, implies that the moduli space $\mathcal{Z}$ of rational curves in $\mathcal{Y}$ is smooth and of dimension $2 k+2$ near $C$. The case where $C$ is not embedded is easily reduced to the embedded case by "separating the crossings of a tubular neighborhood of $C$ ". More precisely, let $\nu: N_{C} \rightarrow \mathcal{Y}$ be the normal exponential mapping with respect to any smooth metric on $\mathcal{Y}$ and let $\tilde{\mathcal{Y}} \subset N_{C}$ denote a neighborhood of the zero section which maps locally diffeomorphically into $\mathcal{Y}$. Give $\tilde{\mathcal{Y}}$ the holomorphic contact structure which makes $\nu$ 
a local contact biholomorphism. Note that the zero section in $\tilde{\mathcal{Y}}$ is an embedded rational contact curve $\tilde{C}$. If $\tilde{\mathcal{Z}}$ denotes the space of unramified rational curves in $\mathcal{Y}$, then $\tilde{\mathcal{Z}}$ is smooth of complex dimension $2 k+2$ near $\tilde{C}$ and $\nu$ clearly induces a biholomorphism of a neighborhood of $\tilde{C}$ in $\tilde{\mathcal{Z}}$ with a neighborhood of $C$ in $Z$.

In view of this, we shall assume for the remainder of the proof that $C$ is embedded.

We will now identify the subspace of $\mathcal{Z}$ near $C$ which consists of contact curves. To do this, we first observe that for any curve $C^{\prime} \subset \mathcal{Y}$, there is a natural pairing $L_{\mid C^{\prime}} \times \tau_{C^{\prime}} \rightarrow \mathbb{C}$ which is the restriction to the given bundles of the natural pairing of co-vectors with vectors. We may regard this pairing as a section $\sigma_{C^{\prime}}$ of the bundle $L_{\mid C^{\prime}}^{*} \times \tau_{C^{\prime}}^{*}$ and note that this section vanishes if and only if $C^{\prime}$ is a contact curve. In the case where $C^{\prime}$ is a rational curve and $L_{\mid C^{\prime}} \simeq \mathcal{O}(-k-1)$, it follows that $\sigma_{C^{\prime}}$ is a section of a line bundle isomorphic to $\mathcal{O}(k-1)$ and hence vanishes identically if and only if it vanishes to order $k$ at some point on $C^{\prime}$.

The rest of our argument requires some slight notational changes in the case $k=0$, so from now on, we will assume that $k \geq 1$, leaving for the reader the task of making those changes needed to make the argument go through for $k=0$. (If $k=0$, then $\sigma_{C^{\prime}} \equiv 0$ anyway since it is a section of a line bundle of negative degree. Thus, it is clear that $\mathcal{M}_{0}=\mathcal{Z}$ in a neighborhood of such a $C$.)

Let $p \in C$ be fixed. Since $C$ is embedded, it follows that we may suppose that there is a neighborhood $U$ of $p$ so that $C \cap U$ consists of a single, analytically irreducible branch of $C$. Moreover, by using a slight extension of the Pfaff-Darboux theorem in the analytic category, we see that it is possible to choose a $p$-centered holomorphic coordinate system $(x, y, z)$ on a $p$-neighborhood $U \subset \mathcal{Y}$ in which $U \cap C$ is described by the equations $y=z=0$ and so that the 1 -form $\theta=d y-z d x$ is a section of $L_{\mid U}$. (The point is that we can choose the Pfaff coordinates so that $C \cap U$ is described in such a simple fashion. To see that this can be done, first choose the $p$-centered coordinate system $(x, y, z)$ so that $\theta=d y-z d x$ is a section of $L_{\mid U}$ and so that $d x$ is a non-vanishing 1-form when pulled back to $C \cap U$. Then, since $C$ is a contact curve, it is easy to see that, by shrinking $U$ if necessary, we may suppose that there exists a holomorphic function $f$ on a neighborhood of $0 \in \mathbb{C}$ so that $C \cap U$ is described by the equations $y=f(x)$ and $z=f^{\prime}(x)$. Replacing the coordinate system $(x, y, z)$ by $\left(x, y-f(x), z-f^{\prime}(x)\right.$ ) (and again possibly shrinking $U$ ), we get the desired new coordinate system.)

By Kodaira's description of the moduli space $\mathcal{Z}$ near $C$, there exist holomorphic functions $y_{j}, z_{j}$ for $j \geq 0$ on a $C$-neighborhood $\mathcal{U} \subset \mathcal{Z}$ so that, for $C^{\prime} \in \mathcal{U}$, we have $L_{\mid C^{\prime}} \simeq \mathcal{O}(-k-1)$ and the defining equations of $U \cap C^{\prime}$ take the form

$$
\begin{aligned}
& y=y_{0}\left(C^{\prime}\right)+y_{1}\left(C^{\prime}\right) x+\cdots+y_{j}\left(C^{\prime}\right) x^{j}+\cdots \\
& z=z_{0}\left(C^{\prime}\right)+z_{1}\left(C^{\prime}\right) x+\cdots+z_{j}\left(C^{\prime}\right) x^{j}+\cdots
\end{aligned}
$$

We want to show that it is possible to select a specific set of $(2 k+2)$ of the functions $y_{j}, z_{j}$ in such a way that, after possibly shrinking $\mathcal{U}$, this set forms a $C$-centered coordinate system on $\mathcal{U}$. In order to do this, we need to use our information about the normal bundle of $C$. By KMT, if $\left\{\nu_{\alpha} \mid 1 \leq \alpha \leq 2 k+2\right\}$ is any basis for the global sections of $N_{C}$, 
then there exists a $C$-centered local coordinate system $\left(t^{1}, t^{2}, \ldots, t^{2 k+2}\right)$ on $\mathcal{U}$ so that, with respect to the local coordinate trivialization of $N_{C}$ over $C \cap U$ determined by $(x, y, z)$, we have

$$
\nu_{\alpha}=\left(\sum_{j \geq 0} \frac{\partial y_{j}}{\partial t^{\alpha}}(C) x^{j}\right)\left[\frac{\partial}{\partial y}\right]+\left(\sum_{j \geq 0} \frac{\partial z_{j}}{\partial t^{\alpha}}(C) x^{j}\right)\left[\frac{\partial}{\partial z}\right]
$$

for all $\alpha$, where [ ] represents reduction modulo the sub-bundle spanned by the vector field $\partial / \partial x$ which spans $\tau$.

Now let us first suppose that $N_{C} \simeq \mathcal{O}(k) \oplus \mathcal{O}(k)$. Then it is clear that there is a basis $\left\{\nu_{\alpha} \mid 1 \leq \alpha \leq 2 k+2\right\}$ for the global sections of $N_{C}$ which is expressed in the coordinate trivialization given above in the form

$$
\begin{aligned}
& \nu_{j+1}=x^{j}\left[\frac{\partial}{\partial y}\right]+O\left(x^{k+1}\right) \\
& \nu_{j+k+2}=x^{j}\left[\frac{\partial}{\partial z}\right]+O\left(x^{k+1}\right) \quad \text { for } \quad 0 \leq j \leq k
\end{aligned}
$$

where the symbol $O\left(x^{k+1}\right)$ denotes terms which vanish to order $(k+1)$ at $x=0$. Comparing (5) and (6), we see that the Jacobian matrix of the functions $y_{0}, y_{1}, \ldots, y_{k}, z_{0}, z_{1}, \ldots, z_{k}$ with respect to the coordinate system $\left(t^{1}, \ldots, t^{2 k+2}\right)$ is the identity matrix at $C$. By the Implicit Function Theorem, it is possible to shrink $\mathcal{U}$ so that $\left(y_{0}, y_{1}, \ldots, y_{k}, z_{0}, z_{1}, \ldots, z_{k}\right)$ forms a $C$-centered holomorphic coordinate system on $\mathcal{U}$.

Alternatively, let us suppose that $N_{C} \simeq \mathcal{O}(k-1) \oplus \mathcal{O}(k+1)$. Then the sequence (2) is canonically split, i.e., there exists a unique holomorphic line bundle $N_{C}^{\prime} \subset N_{C}$ which projects isomorphically onto $L_{C}^{*}$ in the sequence (2). Moreover, $N_{C}^{\prime}$ must be everywhere transverse to $L_{\mid C}^{\perp} / \tau$. Since $L_{\mid C}^{\perp} \cap U$ is clearly spanned by the vector fields $\{\partial / \partial x, \partial / \partial z\}$, it follows that there exists a holomorphic function $f(x)$ for $x$ near $0 \in \mathbb{C}$, so that the expression $[\partial / \partial y+f(x) \partial / \partial z]$ is a local section of $N_{C}^{\prime}$ over $C \cap U$. Now, replacing the coordinate system $(x, y, z)$ by the system $\left(x, g(x) y, g(x) z+g^{\prime}(x) y\right)$ where $g$ is the holomorphic function which satisfies $g(0)=1$ and $g^{\prime}(x)+f(x) g(x)=0$, we retain all of our earlier normalizations and get a new coordinate system in which the coordinate trivialization of $N_{C}^{\prime}$ over $C \cap U$ is spanned by $[\partial / \partial y]$. It is now easy to choose a basis $\left\{\nu_{\alpha} \mid 1 \leq \alpha \leq 2 k+2\right\}$ for the global sections of $N_{C}$ which are expressed in the local coordinate trivialization in the form

$$
\begin{array}{ll}
\nu_{j+1}=x^{j}\left[\frac{\partial}{\partial y}\right]+O\left(x^{k+2}\right) & \text { for } \quad 0 \leq j \leq k+1, \\
\nu_{j+k+3}=x^{j}\left[\frac{\partial}{\partial z}\right]+O\left(x^{k}\right) & \text { for } \quad 0 \leq j \leq k-1,
\end{array}
$$

Again, an application of the Implicit Function Theorem shows that it is possible to shrink $\mathcal{U}$ so that the functions $y_{0}, y_{1}, \ldots, y_{k+1}, z_{0}, z_{1}, \ldots, z_{k-1}$ form a $C$-centered holomorphic coordinate system on $\mathcal{U}$. 
This leads us to the following important observation: In either case, the functions $y_{0}, y_{1}, \ldots, y_{k}, z_{0}, z_{1}, \ldots, z_{k-1}$ have linearly independent differentials on a neighborhood of $C \in \mathcal{U}$.

Since the bundle $L$ has been trivialized over $U$, it follows that for $C^{\prime} \in \mathcal{U}$, the section $\sigma_{C^{\prime}}$ has the local expression

$$
\begin{aligned}
\sigma_{C^{\prime}} & =(d y-z d x)_{\mid C^{\prime}} \\
& =\left(\left(y_{1}\left(C^{\prime}\right)-z_{0}\left(C^{\prime}\right)\right)+\cdots+\left(k y_{k}\left(C^{\prime}\right)-z_{k-1}\left(C^{\prime}\right)\right) x^{k-1}+\cdots\right) d x .
\end{aligned}
$$

Because the functions $y_{0}, y_{1}, \ldots, y_{k}, z_{0}, z_{1}, \ldots, z_{k-1}$ have linearly independent differentials at $C$, the locus $\mathcal{M}_{\mathcal{U}}$ defined by the equations $z_{j-1}-j y_{j}=0$ for $1 \leq j \leq k$ is a smooth submanifold of $\mathcal{Z}$ of codimension $k$ in a neighborhood of $C$. Now note that any $C^{\prime}$ in $\mathcal{M U}_{\mathcal{U}}$ has $\sigma_{C^{\prime}}$ vanishing to order $k$ at at least one point. Since, for $C^{\prime} \in \mathcal{U}$, we know that $\sigma_{C^{\prime}}$ takes values in a line bundle isomorphic to $\mathcal{O}(k-1)$, it follows that any $C^{\prime} \in \mathcal{M}_{\mathcal{U}}$ must have $\sigma_{C^{\prime}} \equiv 0$ and hence is a contact curve. It follows that $\mathcal{M}_{\mathcal{U}}$ is equal to $\mathcal{M}_{k} \cap \mathcal{U}$ and is smooth of dimension $k+2$.

To finish the proof, we must rule out the possibility that the sequence (2) splits. By $\mathrm{KMT}$, if the functions $y_{0}, y_{1}, \ldots, y_{k}, z_{0}, z_{1}, \ldots, z_{k}$ have linearly independent differentials at $C \in \mathcal{U}$, then $N_{C}$ is isomorphic to $\mathcal{O}(k) \oplus \mathcal{O}(k)$. Thus, we proceed to show this independence.

We have seen that $\mathcal{M}_{k} \cap \mathcal{U}$ is defined as an analytic subvariety of $\mathcal{U}$ by the vanishing of the (independent) functions $f_{j-1}=z_{j-1}-j y_{j}$ for $1 \leq j \leq k$. Moreover, since $d y-z d x$ vanishes identically on any contact curve, it follows that $f_{k}=z_{k}-(k+1) y_{k+1}$ also vanishes on $\mathcal{M}_{k} \cap \mathcal{U}$. Since the functions $f_{0}, \ldots, f_{k-1}$ are a regular defining ideal for $\mathcal{M}_{k}$ near $C$, the function $f_{k}$ must be in the ideal defined by these functions so

$$
f_{k}=a^{0} f_{0}+a^{1} f_{1}+\cdots+a^{k-1} f_{k-1}
$$

for some local holomorphic functions $a^{j}$. It follows that at $C$, we have a relation of the form

$$
d z_{k}=(k+1) d y_{k+1}+a^{0}\left(d z_{0}-d y_{1}\right)+\cdots+a^{k-1}\left(d z_{k-1}-k d y_{k}\right)
$$

which implies that at $C$, we have

$$
d z_{k} \equiv(k+1) d y_{k+1} \quad \text { modulo } \quad d y_{0}, d y_{1}, \ldots, d y_{k}, d z_{0}, d z_{1}, \ldots, d z_{k-1}
$$

Thus, the functions $y_{0}, y_{1}, \ldots, y_{k}, z_{0}, z_{1}, \ldots, z_{k}$ have linearly independent differentials at $C$ if and only if the functions $y_{0}, y_{1}, \ldots, y_{k+1}, z_{0}, z_{1}, \ldots, z_{k-1}$ have linearly independent differentials at $C$. Since, by our above arguments, at least one of these two sets of functions must have linearly independent differentials at $C$, it follows that they both do. In particular, the first set does, as we wished to show.

In this paper, the important case is $k=2$ for then we have the following theorem:

Theorem 5.2: Let $\mathcal{Y}$ be a contact 3-fold and let $\mathcal{M}$ be the moduli space consisting of those rational contact curves $C \subset \mathcal{Y}$ which satisfy $L_{\mid C} \simeq \mathcal{O}(-3)$. Then $\mathcal{M}$ is a smooth manifold of dimension 4. If we let $\mathcal{N} \subset \mathcal{M} \times \mathcal{Y}$ be the incidence submanifold consisting 
of pairs $(C, p) \in \mathcal{M} \times \mathcal{Y}$ such that $p \in C$, and let $\mathrm{l}: \mathcal{N} \rightarrow \mathcal{M}$ and $\mathrm{r}: \mathcal{N} \rightarrow \mathcal{Y}$ denote the projections onto the first and second factors, then the diagram

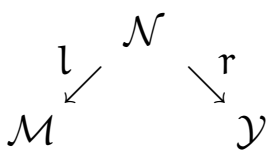

is a non-degenerate double fibration which is a generalized contact path geometry. Moreover, the primary and secondary invariants of this geometry vanish, so that there is induced on $\mathcal{M}$ a torsion-free $\mathcal{G}_{3}$-structure.

Moreover, every real-analytic torsion-free $G_{3}$-structure on a 4-manifold $M$ is locally a real slice of a torsion-free $\mathcal{G}_{3}$-structure which arises in the above manner.

Proof: The smoothness of $\mathcal{M}$ has already been demonstrated. In order to show the non-degeneracy of the double fibration, we resort to local coordinates.

FIrst, we show that we can take local coordinates of a particularly simple kind. Let $(C, p) \in \mathcal{N}$ be fixed. Let $U \subset \mathcal{Y}$ be a $p$-neighborhood on which there exist $p$-centered coordinates $(x, y, z)$ as in the proof of Proposition 5.1. In particular, the bundle $L_{\mid U}$ has $\theta=d y-z d x$ as a trivializing section and $C \cap U$ is described by the equations $y=z=0$. Let $\mathcal{U} \subset \mathcal{M}$ be a $C$-neighborhood with the property that $C^{\prime} \cap U \neq \emptyset$ for all $C^{\prime} \in \mathcal{U}$ and so that there exist functions $y_{j}, z_{j}$ so that the defining equations of $C^{\prime} \cap U$ are given by (4) as before. Since the normal bundle of $C$ in $\mathcal{Y}$ is $\mathcal{O}(2) \oplus \mathcal{O}(2)$, it follows that the functions $y_{0}, y_{1}, y_{2}, z_{0}, z_{1}, z_{2}$ contain four functions which form a coordinate system on a neighborhood of $C \in \mathcal{M}$. However, $\mathcal{M}$ is defined as a locus in $\mathcal{Z}$ by the equations $z_{0}=y_{1}=z_{1}-2 y_{2}=0$, so it follows that the set $y_{0}, y_{1}, y_{2}, z_{2}$ must be a local coordinate system on $\mathcal{U}$ once $\mathcal{U}$ has been shrunk sufficiently. Since we also have $z_{j-1}=j y_{j}$ for all $j \geq 1$, it follows that we can equally well take $y_{0}, y_{1}, y_{2}, y_{3}$ as our local coordinate system near $C$, so we do so.

If we let $F$ denote the function of $\left(x, y_{0}, y_{1}, y_{2}, y_{3}\right)$ which is represented by the series on the right hand side of the first equation in (4), then it follows that $\mathcal{N} \cap(\mathcal{U} \times U)$ is defined in $(\mathcal{U} \times U)$ by the equations

$$
y-F=z-\partial F / \partial x=0 .
$$

By shrinking $\mathcal{U}$ and $U$ if necessary, we may suppose that the functions $x, p^{0}=F$, $p^{1}=\partial F / \partial x, p^{2}=\partial^{2} F / \partial x^{2}$, and $p^{3}=\partial^{3} F / \partial x^{3}$ form a local coordinate system $\psi=$ $\left(x, p^{0}, p^{1}, p^{2}, p^{3}\right)$ on $\mathcal{N} \cap(\mathcal{U} \times U)$. In particular, there is a function $\Phi$ defined on a neighborhood of $0 \in \mathbb{C}^{5}$ for which we have

$$
\partial^{4} F / \partial x^{4}=\Phi\left(x, p^{0}, p^{1}, p^{2}, p^{3}\right) .
$$

It follows easily that the semi-basic forms for the projection $l$ are spanned by $\left\{d p^{0}-\right.$ $\left.p^{1} d x, d p^{1}-p^{2} d x, d p^{2}-p^{3} d x, d p^{3}-\Phi \circ \psi d x\right\}$ while the semi-basic forms for the projection $\mathrm{r}$ are spanned by $\left\{d x, d p^{0}, d p^{1}\right\}$.

Since the point $(C, p) \in \mathcal{N}$ was chosen arbitrarily, it immediately follows that the double fibration is non-degenerate and represents a generalized contact path geometry where 
the $\Gamma$-structure is defined locally by the Pfaffian system $\left\{d p^{0}-p^{1} d x, d p^{1}-p^{2} d x, d p^{2}-\right.$ $\left.p^{3} d x\right\}$ and the fibers of $l$ give the foliation by admissible integral curves of this system.

Now, we must show that the primary and secondary invariants of the foliation by l-fibers vanish on $\mathcal{N}$. First, by the structure equations and the remarks in $\S 4$, we know that $\mathcal{A}$ is a holomorphic section of a line bundle on $\mathcal{N}$ and restricts to each fiber of $l$ to be a section of the third power of the canonical bundle of the fiber. Since these fibers are rational curves, there are no non-zero holomorphic sections of any positive power of the canonical bundle. Thus, $\mathcal{A}=0$. The secondary invariant $\mathcal{B}$ is now well-defined and restricts to each fiber of $l$ to be a holomorphic section of the fourth power of the canonical bundle. Thus, $\mathcal{B}=0$.

Let $\hat{L}$ denote the pull-back of the line bundle $L$ to $\mathcal{N}$. Note that $\mathcal{C}$ is a holomorphic section of a line bundle on $\mathcal{N}$ and that this line bundle restricts to each fiber of $l$ to be a holomorphic section of the tensor product of $\hat{L}$ and the canonical bundle of the fiber. Now $\hat{L}$ restricts to each fiber of $l$ to be isomorphic to $\mathcal{O}(-3)$, so it follows that $\mathcal{C}$ is a section of a line bundle isomorphic to $\mathcal{O}(-5)$. Thus, $\mathcal{C}=0$. In particular, $\mathcal{D}$ is well-defined. Finally, arguing as above, $\mathcal{D}$ restricts to each fiber of $l$ to be a section of a line bundle isomorphic to $\mathcal{O}(-7)$. Thus, $\mathcal{D}=0$.

Of course, by the holomorphic version of Theorem 4.5, we now have the desired torsionfree $\mathcal{G}_{3}$-structure on $\mathcal{M}$.

The proof of the final statement of the theorem, that every real-analytic torsion-free $G_{3}$-structure is a real slice of a torsion-free $\mathcal{G}_{3}$-structure arising in the above manner, proceeds as expected, except for one point which we will now explain.

If we suppose that the torsion-free $G_{3}$-structure $F$ on $M$ is real analytic in appropriate local coordinate systems, then by restricting to sufficiently small open sets in $M$, we may suppose that $M$ is the real slice of a complex manifold $\mathcal{M}$ endowed with a real structure (i.e., an anti-holomorphic involution whose fixed point set is $M$ ) and that $F$ is the real slice of an appropriate $\mathcal{G}_{3}$-structure $\mathcal{F}$ on $\mathcal{M}$. It easily follows that $\mathcal{F}$ is torsion-free and, moreover, by restricting to an even smaller open set in $M$ if necessary, we may assume that $\mathcal{F}$ is amenable, so that we get a double fibration as in the statement of the theorem. Note that we only have to localize in $M$. In particular, it follows that $\mathcal{Y}$ is a complex contact 3 -fold and that each of the curves $\mathcal{R}_{x}$ for $x \in \mathcal{M}$ is a rational contact curve.

To finish the proof, we need to see why the restriction of the contact bundle $L$ to $\mathcal{R}_{x}$ is isomorphic to $\mathcal{O}(-3)$. However, this follows directly from the complex versions of the structure equations (ii) of Proposition 4.4.

Of course, it is possible to describe the $\mathcal{G}_{3}$-structure on $\mathcal{M}$ directly in terms of the incidence geometry of the family of rational curves in $\mathcal{Y}$. For example, the set of curves in $\mathcal{M}$ which meet a given curve $C \in \mathcal{M}$ constitutes the set of points which are "nullseparated" from $C$ in the sense determined by the conformal quartic form on $\mathcal{M}$ which determines the $\mathcal{G}_{3}$-structure. The set of curves in $\mathcal{M}$ which are tangent to a given curve $C \in \mathcal{M}$ constitutes the set of points which are "null-separated" from $C$ in the sense determined by the field of rational normal cones on $\mathcal{M}$ which determines the $\mathcal{G}_{3}$-structure (see $\S 4$ ). In particular, the geodesics in $\mathcal{M}$ whose tangent vectors are "perfect cubes" are 
in one-to-one correspondence with the 1-parameter families of rational curves in $\mathcal{Y}$ which pass through a given point in a given direction.

A direct proof that this $\mathcal{G}_{3}$-structure is torsion-free can be based on the holomorphic analogue of Theorem 4.1, using the fact that the 2-parameter subfamily of curves which pass through a fixed point in $\mathcal{Y}$ describes a null surface in $\mathcal{M}$.

We now come to the interesting question of how useful Theorem 5.2 is in proving existence, constructing examples, or discussing the generality of torsion-free $\mathcal{G}_{3}$-structures on complex 4 -folds $\mathcal{M}$.

First, we show that Theorem 5.2 is not vacuous. We will describe a class of complex contact 3 -folds which contain rational contact curves to which $L$ restricts to become isomorphic to $\mathcal{O}(-3)$.

Let $\mathcal{S}$ be a complex surface and let $\mathcal{Y}=\mathbb{P}(T \mathcal{S})$ be the projectivized holomorphic tangent bundle of $\mathcal{S}$. It is easy to show that $\mathcal{Y}$ has a canonical contact line bundle $L \subset T^{*} \mathcal{Y}$ defined as follows: Let $\ell \subset T_{s} \mathcal{S}$ be a line and define $L_{\ell} \subset T_{\ell}^{*} \mathcal{Y}$ to be the line $\pi^{*}\left(\ell^{\perp}\right)$ where $\pi: \mathcal{Y} \rightarrow \mathcal{S}$ is the base-point projection and $\ell^{\perp} \subset T_{s}^{*} \mathcal{S}$ is the annihilator of $\ell$.

Any curve $C \subset \mathcal{S}$ has a canonical lift to $\mathcal{Y}$ as a contact curve; this lift being defined by sending $p \in C$ to $T_{p} C$. (If $C$ is ramified, this lifting extends holomorphically across the ramification points. In fact, it "resolves" simple cusps.) Conversely, it is easy to show that every irreducible contact curve in $\mathcal{Y}$ is either a fiber of $\pi$ or else is the canonical lift of an irreducible curve in $\mathcal{S}$.

From our description, it is clear that if $C \subset \mathcal{S}$ is an unramified rational curve with normal bundle $\mathcal{O}(k+1)$, then, as a curve in $\mathcal{Y}$, it satisfies $L_{\mid C} \simeq \mathcal{O}(-k-1)$. If, in addition, $k \geq 0$, then Proposition 5.1 shows that $C$ belongs to a $(k+2)$-parameter family of rational contact curves. Of course, the projection of these curves back into $\mathcal{S}$ agrees with the moduli space of curves near $C$ in $\mathcal{S}$.

Taking $k=2$ in this construction, we obtain examples of the desired contact 3 -folds.

A specific example is obtained by letting $\mathcal{S}=\mathcal{O}(3)$, i.e., regarding the bundle $\mathcal{O}(3)$ over $\mathbb{P}^{1}$ as a surface. Then, taking $C$ to be the zero-section of $\mathcal{O}(3)$, we get a rational curve $C$ with the normal bundle $\mathcal{O}(3)$. However, this example is not too interesting because the moduli space $\mathcal{M}$ of nearby contact curves in this case is just the space of global sections of $\mathcal{O}(3)$ itself, which forms a vector space of dimension 4 . The corresponding $\mathcal{G}_{3}$-structure is easily seen to be the flat one on $\mathbb{C}^{4}$.

A more interesting class of examples is to take a rational curve $C$ in $\mathbb{P}^{2}$ of degree $d \geq 2$ whose only singularities are $D=(d-1)(d-2) / 2$ distinct nodes. (This is true for the generic rational curve of degree $d$ in $\mathbb{P}^{2}$.) The nearby rational curves form a $(3 d-1)$ parameter family. Select a set $S$ consisting of $s$ smooth points on $C$ and a set $N$ consisting of $n$ nodes on $C$ where $s+2 n=3 d-5$. Let $\mathcal{S}$ be the surface got from $\mathbb{P}^{2}$ by blowing up the points in $S \cup N$. Then the normal bundle of $C$ as a curve in $\mathcal{S}$ is isomorphic to $\mathcal{O}(3)$. It follows that the moduli space of nearby rational curves in $\mathcal{S}$ carries a canonical torsion-free $\mathcal{G}_{3}$-structure.

The specific case of a non-singular conic in $\mathbb{P}^{2}$ with one point blown up yields the 4-parameter family of conics passing through a single point. Thus, this family of curves 
can be described in the form

$$
\left(x+p_{3}\right) y+p_{2} x^{2}+p_{1} x+p_{0}=0 .
$$

The corresponding fourth order differential equation is easily seen to be $y^{(4)}=\frac{4}{3}\left(y^{\prime \prime \prime}\right)^{2} / y^{\prime \prime}$. It is not difficult to show that the corresponding $\mathcal{G}_{3}$-structure is not flat. In fact, the holonomy is $\mathcal{H}_{3}$ and the $\mathcal{H}_{3}$-structure is (the complexification of) the unique non-flat homogeneous structure $F^{0}$ discussed in $\S 3$.

It is an interesting question as to how general a $\mathcal{G}_{3}$-structure can be constructed by varying $d$ and the sets $S$ and $N$. Not all of these are distinct since, for example, the conic with one point blown up and the nodal cubic with its node and two smooth points blown up give rise to isomorphic $\mathcal{G}_{3}$-structures. More generally, any two such 4 -parameter families of rational curves which differ by a Cremona transformation of $\mathbb{P}^{2}$ will give rise to isomorphic $\mathcal{G}_{3}$-structures.

Finally, once one example of a rational contact curve $C$ with normal bundle $N_{C} \simeq$ $\mathcal{O}(2) \oplus \mathcal{O}(2)$ in a contact manifold $\mathcal{Y}$ has been constructed, the Spencer-Kodaira theory of deformation of pseudo-group structures may be applied. Thus, one expects to be able to construct more examples by deforming a neighborhood of $C$ in $\mathcal{Y}$ as a complex contact manifold. In fact, Kodaira [1960] shows that the tangent space to the moduli of nearby complex contact structures on a tubular neighborhood $U$ of $C$ in $\mathcal{Y}$ is given by $H^{1}\left(U, \Theta_{c}\right)$ where $\Theta_{c} \subset \Theta$ is the sheaf of contact vector fields, i.e., the vector fields whose infinitesimal flows preserve the bundle $L$.

Now, on any complex contact manifold $\mathcal{Y}$ with contact line bundle $L \subset T^{*} \mathcal{Y}$, the sheaf of contact vector fields is isomorphic over $\mathbb{C}$ to the sheaf of sections of $L^{*}$. The reason for this is that the sheaf of $\mathcal{O}$-modules

$$
0 \longrightarrow L^{\perp} \longrightarrow T \longrightarrow L^{*} \longrightarrow 0
$$

has a $\mathbb{C}$-splitting given by a first order differential operator $D_{0}: L^{*} \rightarrow T$ with the property that a vector field $X$ on $U \subset \mathcal{Y}$ is a local contact vector field if and only if $X=D_{0}([X])$ where [] denotes reduction modulo $L^{\perp}$.

Since $L^{*}$ restricts to $C$ to be isomorphic to $\mathcal{O}(3)$, it follows that, if $U$ is a sufficiently small tubular neighborhood of $C$ in $\mathcal{Y}$, then $H^{1}\left(U, \Theta_{c}\right)$ will be "large". Thus, one expects there to be many non-trivial local contact deformations of this neighborhood.

It is not hard to see that under small deformations of $U$, the 4-parameter family of rational contact curves persists. Essentially, this is because the normal bundle of $C$ is positive, see Kodaira [1963]. However, some care must be taken in this argument since we want contact curves, so just Kodaira stability is not quite sufficient.

We shall not go into any detail about this deformation theory here because we have already seen by other methods in $\S 3$ that the general local real analytic solutions in the real category depend on four arbitrary analytic functions of three variables. It would be interesting to see whether there is a direct relation between the description of the generality of local solutions via the Cartan-Kähler theory and the description of the same "moduli space" by Kodaira-Spencer deformation theory. 


\section{$\S 6$. Epilogue}

The function of this short last section is to collect together some of the open problems and questions which this investigation has raised.

The fundamental underlying problem from $\S 1$ is, of course, the classification of the possible holonomy groups of affine symmetric connections. In particular, what are the remaining exotic affine holonomies? Perhaps if we knew some of the methods which enter into the proof of Berger's Theorem 4, we could determine a finite inclusive list and then examine each one separately.

The methods of $\S 3$ give no information about degenerate torsion-free $G_{3}$-structures. Since the symmetry group of a non-degenerate torsion-free $G_{3}$-structure is easily seen to be discrete, it follows that Theorem 3.3 can say nothing about possible homogeneous examples. Indeed, we do not know if any homogeneous examples exist other than the flat structure or the unique homogeneous example with holonomy $H_{3}$.

Also, the issues of completeness of the intrinsic connection and/or global amenability are completely ignored in our treatment. Even in the case where the holonomy is $H_{3}$, we do not know anything about the global nature of the $H_{3}$-structures. In particular, note that Theorem 3.4 does not give any global information.

For example, is it reasonable to conjecture that each connected component of a level set of $\mathbf{R}_{c}$ in $\mathcal{V} \backslash \Sigma_{c}$ corresponds to a connected smooth 4-manifold endowed with a complete $H_{3}$-structure? While it seems reasonable that one should be able to "piece together" the local solutions corresponding to open sets in the level set to get a connected 4-manifold, the resulting object might be neither Hausdorff nor smooth. In fact, it seems quite likely that solutions may develop orbifold-type singularities (or worse) under this patching process. One source of trouble for this approach is that each such local solution has a local 1parameter symmetry group (which may even have fixed points), so there is no a priori canonical way to identify solutions on "overlaps".

One obstruction to globalizing the proof of Theorem 3.4 is that, at a crucial point in the argument, a closed 2 -form $\bar{\Psi}$ is assumed to be exact. Of course, this cannot be done globally if $\bar{\Psi}$ represents a non-trivial element in the second deRham cohomology group of the stratum. Unfortunately, due to the complexity of the polynomial function $\mathbf{R}_{c}$, it does not appear to be easy to deduce anything about the topology of its level sets. Thus, determining the deRham class of $\bar{\Psi}$ seems to be difficult.

Nothing is known about the torsion-free $H_{3}$-structures which have a three-parameter symmetry group. These correspond to the irreducible components of the singular locus $\Sigma_{c}$. For $c \neq 0$, there are two of these components. While one of these components is rational, the other does not seem to be (although we have no proof of this).

In $\S 4$, a geometric interpretation of the primary and secondary invariants of generalized contact path geometries is entirely lacking. We only know an interpretation of the vanishing of those invariants. Since this geometry is essentially equivalent to the geometry of fourth order ODE in the plane up to contact equivalence, some interpretation of these invariants should be possible in terms of classical ODE. 
A sample question is: What are the conditions on a fourth order ODE which characterize the Euler-Lagrange equations of second order functionals for curves in the plane? These conditions, which are analogous to "Helmholtz conditions with multipliers," should be expressible in terms of the invariants we have derived.

Of course, this particular question should be easy to answer by the method of equivalence. It may even be possible to do this by comparing our calculations with Cartan's calculations in Cartan [1936]. Nevertheless this serves to illustrate what sort of problems there are to consider.

In $\S 5$, we have given no discussion of how one might explicitly incorporate reality conditions into the construction so that one can see the relationship between the real analytic and complex settings explicitly. The reality condition is, of course, important for computation and indicates interesting differences between this theory and the analogous theory of Penrose for the non-linear graviton.

Our construction associates a complex twistor space to each of the local real analytic $G_{3}$-holonomy affine connections while Penrose's construction associates a complex twistor space to each self-dual or anti-self-dual solution to Einstein's equations.

On the other hand, Penrose's construction works for pseudo-metrics of two distinct signatures, $(4,0)$ and $(2,2)$. Our construction works for only one of the real forms of $\mathcal{G}_{3}$. In fact, the other real forms of $\mathcal{G}_{3}$ in this representation cannot occur as the affine holonomy of a symmetric connection as they do not satisfy Berger's two conditions.

One consequence of Proposition 5.1 is that a holomorphic torsion-free $\mathcal{G}_{3}$-structure on a complex 4-manifold $\mathcal{M}$ induces a local embedding of $\mathcal{M}$ into a certain complex 6manifold, $\mathcal{Z}$. This embedding is constructed by interpreting the points of $\mathcal{M}$ as rational contact curves in a contact 3 -fold $\mathcal{Y}$ and then regarding $\mathcal{Z}$ as the full space of rational curves in $\mathcal{Y}$ with normal bundle $\mathcal{O}(2) \oplus \mathcal{O}(2)$.

Since the tangent space at each point of $\mathcal{Z}$ is isomorphic to $\mathbb{C}^{2} \otimes H^{0}(\mathcal{O}(2))$, it is apparent that $\mathcal{Z}$ has a canonical $\mathrm{GL}(2, \mathbb{C}) \otimes \mathrm{SO}(3, \mathbb{C})$-structure. For twistorial reasons, it seems likely that the intrinsic torsion of this structure vanishes. If so, it is also quite likely that $\mathrm{GL}(2, \mathbb{C}) \otimes \mathrm{SO}(3, \mathbb{C}) \subset \mathrm{GL}(6, \mathbb{C})$ is yet another exotic affine holonomy group. The relationship between the geometry on $\mathcal{Z}$ and that on $\mathcal{M}$ is far from understood.

Finally there is the question of characterizing the complex contact manifolds which correspond to the finite dimensional family of affine connections with holonomy $\mathcal{H}_{3}$. These should be some special complex manifolds which have at least a one-parameter symmetry group. Other than the homogeneous case that we discussed at the end of $\S 3$ and $\S 5$, none of the other examples are known. In particular, the complex contact manifolds corresponding to the two components of the singular locus $\Sigma_{c}$ should have 3-parameter symmetry groups and may be "almost homogeneous", but so far, they have resisted explication.

Department of Mathematics

MARCH 1990

DUKE UNIVERSITY

Durham, NC 27706

BRYANT@MATH.DUKE.EDU 


\section{Bibliography}

M. Berger, Sur les groupes d'holonomie des variétés à connexion affine et des variétés Riemanniennes, Bull. Soc. Math. France, 83 (1955), 279-330.

A. Besse, Einstein Manifolds, Ergebnisse der Mathematik und ihrer Grenzgebiete 3. Folge, Band 10, Springer-Verlag, 1987.

R. Bryant, Metrics with exceptional holonomy, Annals of Mathematics, 126 (1987), $525-576$.

R. Bryant, S.S. Chern, R. Gardner, H. Goldschmidt, and P. Griffiths Exterior Differential Systems, MSRI Series, Springer-Verlag, Berlin, New York, 1990.

É. Cartan, Sur la structure des groupes infinis de transformations, Annales École Normale, 21 (1904), 153-206.

É. Cartan, La géométrie de l'intégrale $\int F\left(x, y, y^{\prime}, y^{\prime \prime}\right) d x$., J. Math. pures et appl., 15 (1936), 42-69.

É. Cartan, Les espaces généralisés et l'intégration de certaines classes d'équations différentielles, C. R. Acad. Sc., 206 (1938), 1689-1693.

É. Cartan, La geometria de las ecuaciones diferenciales de tercer orden, Revista. Mat. Hispano-Amer., 4 (1941), 1-31.

É. Cartan, Sur une classe d'espaces de Weyl, Annales École Normale, 60 (1943), 1-16.

S.S. Chern, The geometry of the differential equation $y^{\prime \prime \prime}=F\left(x, y, y^{\prime}, y^{\prime \prime}\right)$, Science Reports Tsing Hua Univ., 4 (1940), 97-111. (Reprinted in Shiing-shen Chern: Selected Papers, vol. I, Springer-Verlag, New York, 1978)

R. Gardner, The Method of Equivalence and its Applications, CBMS-NSF Regional Conference Series in Applied Mathematics 58, SIAM, Philadelphia, 1989.

N. Hitchin, Complex manifolds and Einstein's equations, in Twistor Geometry and NonLinear Systems, ed. H.D. Doebner and T.D. Palev, Lecture Notes in Mathematics, No. 970, Springer-Verlag, Berlin, 1982, 73-99.

J. Humphreys, Introduction to Lie Algebras and Representation Theory, Graduate Texts in Mathematics no. 9, Springer-Verlag, 1972.

S. Kobayashi and K. Nomizu, Foundations of Differential Geometry, Wiley Interscience, New York, vols. 1 and 2, 1963.

K. Kodaira, On deformations of some complex pseudo-group structures, Annals of Mathematics, 71 (1960), 224-302.

K. Kodaira, A theorem of completeness of characteristic systems for analytic families of compact submanifolds of complex manifolds, Annals of Mathematics, 75 (1962), 146-162.

K. Kodaira, On deformations of some complex pseudo-group structures, Amer. J. of Math., 85 (1963), 79-94.

C. LeBrun, Spaces of complex null geodesics in complex Riemannian geometry, Trans. Amer. Math. Soc., 278 (1983), 209-231.

S. Salamon, Riemannian geometry and holonomy groups, Pitman Research Notes in Mathematics, no. 201, Longman Scientific \& Technical, Essex, 1989. 